\title{
A Systematic Review on Fitness Testing in Adult Male Basketball Players: Tests Adopted, Characteristics Reported and Recommendations for Practice
}

\author{
Matthew Morrison ${ }^{1} \cdot$ David T. Martin ${ }^{1} \cdot$ Scott Talpey $^{2} \cdot$ Aaron T. Scanlan $^{3} \cdot$ Jace Delaney $\cdot$ Shona L. Halson ${ }^{1,4}$. \\ Jonathon Weakley ${ }^{1,4,5}$ (1)
}

Accepted: 8 December 2021 / Published online: 4 February 2022

(c) The Author(s) 2022

\begin{abstract}
Background As basketball match-play requires players to possess a wide range of physical characteristics, many tests have been introduced in the literature to identify talent and quantify fitness in various samples of players. However, a synthesis of the literature to identify the most frequently used tests, outcome variables, and normative values for basketball-related physical characteristics in adult male basketball players is yet to be conducted.

Objective The primary objectives of this systematic review are to (1) identify tests and outcome variables used to assess physical characteristics in adult male basketball players across all competition levels, (2) report a summary of anthropometric, muscular power, linear speed, change-of-direction speed, agility, strength, anaerobic capacity, and aerobic capacity in adult male basketball players based on playing position and competition level, and (3) introduce a framework outlining recommended testing approaches to quantify physical characteristics in adult male basketball players.

Methods A systematic review of MEDLINE, PubMed, SPORTDiscus, Scopus, and Web of Science was performed following the Preferred Reporting Items for Systematic Reviews and Meta-Analyses guidelines to identify relevant studies. To be eligible for inclusion, studies were required to: (1) be original research articles; (2) be published in a peer-reviewed journal; (3) have full-text versions available in the English language; and (4) include the primary aim of reporting tests used and/or the physical characteristics of adult (i.e., $\geq 18$ years of age) male basketball players. Additionally, data from the top $10 \mathrm{draft}$ picks who participated in the National Basketball Association combined from 2011-12 to 2020-21 were extracted from the official league website to highlight the physical characteristics of elite 19- to 24-year-old basketball players.

Results A total of 1684 studies were identified, with 375 being duplicates. Consequently, the titles and abstracts of 1309 studies were screened and 231 studies were eligible for full-text review. The reference list of each study was searched, with a further 59 studies identified as eligible for review. After full-text screening, 137 studies identified tests, while 114 studies reported physical characteristics in adult male basketball players.

Conclusions Physical characteristics reported indicate a wide range of abilities are present across playing competitions. The tests and outcome variables reported in the literature highlight the multitude of tests currently being used. Because there are no accepted international standards for physical assessment of basketball players, establishing normative data is challenging. Therefore, future testing should involve repeatable protocols that are standardised and provide outcomes that can be monitored across time. Recommendations for testing batteries in adult male basketball players are provided so improved interpretation of data can occur. Clinical Trial Registration This review was registered with the International Prospective Register of Systematic Reviews and allocated registration number CRD42020187151 on 28 April, 2020.
\end{abstract}

\section{Introduction}

Basketball has been reported by The Fédération Internationale de Basketball (FIBA) as the second most popular sport in the world [1]. The duration of a game varies depending

Extended author information available on the last page of the article on the governing body or federation, level of competition, as well as the age and sex of players [2]. However, the typical format for adult male matches are two 20-min halves (e.g., National Collegiate Athletic Association [NCAA]), four 10-min quarters (e.g., FIBA match-play), or four 12-min quarters (e.g., National Basketball Association [NBA]) [2]. Basketball is typically played on a wooden court with 


\section{Key Points}

Success in basketball is predicated on players optimising multiple basketball-specific skills, which are influenced by many different physical characteristics. As a result, numerous tests have been introduced for the purposes of identifying talent and quantifying fitness across various samples of adult male players.

The wide range of tests and outcome variables reported in the literature illustrates the need to identify (a) physical characteristics that are most important for optimal match performance and (b) the most suitable tests and outcome variables for quantifying physical characteristics of interest.

Tests that are most suitable to identify talent may differ from tests that are most suitable for tracking changes in fitness and fatigue

Future research should focus on developing standardised testing batteries in conjunction with the International Basketball Federation and national governing bodies that contribute to meaningful normative data. A large international data set will facilitate an understanding of historical trends and allow basketball practitioners to become familiar with minimum and desirable fitness standards for their players.

playing areas of $28.7 \mathrm{~m} \times 15.2 \mathrm{~m}(\mathrm{NBA})$ or $28 \mathrm{~m} \times 15 \mathrm{~m}$ (FIBA). Basketball teams consist of up to 12 players per team with five players competing for each team on the court at any one time during matches. The traditional five on-court playing positions include point guard, shooting guard, small forward, power forward, and centre, which are often categorised as backcourt (i.e. point guard and shooting guard) and frontcourt players (i.e., small forward, power forward, and centre) $[3,4]$.

The physical demands of a basketball game have been readily investigated [5-14]. Given the intermittent nature and varying positional demands involved in basketball match-play, a range of well-developed physical characteristics are thought to be required by basketball players [15-19]. During basketball matches, players are required to cover distances between 4400 and $7500 \mathrm{~m}$, which consists predominantly of jogging, sprinting, jumping and changes in direction [12]. While frequently reaching speeds in excess of $7 \mathrm{~m} \cdot \mathrm{s}^{-1}$ professional backcourt and frontcourt players have been reported to perform (mean \pm standard deviation) $42 \pm 6$ and $56 \pm 2$ jumps per game, respectively [20]. Furthermore, players of all positions are required to execute shuffling movements at varied intensities while defending opposing players [12] and must be able to quickly identify and respond to the movements of opponents, challenging their agility, lateral movement and acceleration capabilities [21]. Although basketball is considered a non-collision sport, players will often block, push, and compete for possession with one another as they attempt to create and defend space on the court. The complex nature of basketball match-play clearly indicates the development of multiple physical characteristics can be advantageous to optimise match performance. However, it is important to be able to measure these physical characteristics independently of skill as physical capacities and skill often require different training stimuli to develop.

To assess the physical characteristics of basketball players, it is essential that tests are valid and reliable to ensure basketball practitioners can use the data to make informed decisions regarding training prescription, guiding return to play processes following injury, quantifying individual player progression, profiling and ranking players, and monitoring player performance and fatigue [22-26]. Researchers and practitioners often implement a diverse combination of tests that assess general physical characteristics (e.g., linear sprint speed) $[4,27,28]$, as well as specialised tests that integrate sport-specific skills aimed to replicate certain basketball-specific demands (e.g., dribbling speed tests) [29-31]. However, the wide variety of tests and methods implemented can make it difficult to compare the physical characteristics of adult male basketball players within and between different competition levels. The array of available testing options makes it difficult to understand the physical characteristics required for successful performance in adult male basketball players. Therefore, to help support the quantification and comparison of physical characteristics in adult male basketball players, it is important to identify the most important and desirable characteristics for match performance and report the most common tests and outcome variables used to assess the physical characteristics.

We are unaware of any study that has provided a comprehensive analysis of tests and outcome variables used to assess the physical characteristics of adult male basketball players across all playing levels and positions. While Ziv and Lidor [32] reviewed the physical characteristics of professional male and female basketball players, over a decade has passed since this review was published and substantial growth in the basketball literature science has since occurred. Additionally, Mancha-Triguero et al. [33] reviewed tests used to assess the physical characteristics of high-level male and female players but the range of tests reported were limited with outcome data from each test not provided. Consequently, no review exists examining the tests used and the physical characteristics reported in combination in adult male basketball players from a range of competition levels. Given the world-wide popularity of basketball, it is prudent 
to review the tests used to quantify the physical characteristics of adult male basketball players across different competition levels. Due to the extensive evidence available on male basketball players, it is important to consolidate the current literature for basketball researchers and practitioners alike to develop a clear understanding of current practices in this population. A summary of basketball tests can support basketball practitioners when making decisions based on test results. Furthermore, larger samples of normative data aggregated across studies can lead to comprehensive profiling and benchmarking of important physical characteristics in adult male basketball players. Therefore, the purpose of this review is three-fold, (1) to identify tests and outcome variables used to assess physical characteristics in adult male basketball players across all competition levels, (2) to report a summary of anthropometric, muscular power, linear speed, change-of-direction speed, agility, strength, anaerobic capacity, and aerobic capacity in adult male basketball players based on playing position and competition level, and (3) to introduce a framework outlining recommended testing approaches to quantify physical characteristics in adult male basketball players.

\section{Methods}

\subsection{Design and Search Strategy}

A systematic review was conducted following the Preferred Reporting Items of Systematic Reviews and Meta-Analyses (PRISMA) statement [34]. This review was registered with PROSPERO (ID: CRD42020187151). The academic databases MEDLINE, PubMed, SPORTDiscus, Scopus, and Web of Science were searched from the earliest record until August 2020 to identify English-language, peer-reviewed, original research studies that investigated the tests used and/ or physical characteristics of adult male basketball players. Studies were identified by searching key terms shown in Table 1. Search levels 1-4 were all linked by the Boolean operator 'AND'. Search terms within each search level were joined with 'OR'. When searching the PubMed and
MEDLINE databases 'young adults 19-24 years' and 'adults 19-44 years' limiters were applied to the population age. No limiters were available to be used when searching Web of Science or SPORTDiscus. All search results were extracted and imported to reference manager software (EndNote X9; Thomson Reuters, Philadelphia, PA, USA).

\subsection{Assessment of Reporting Quality}

The methodological quality of each study was assessed using a modified version of the Downs and Black checklist (Table 1 of the Electronic Supplementary Material [ESM]). This checklist has been used previously in systematic reviews related to sport science $[35,36]$ and is a valid method of assessing the quality of studies with observational study designs [37]. The modified version of the Downs and Black checklist was used because the included questions and criteria better align with the specific aims of this review compared with the traditional version of the checklist. The assessment included 12 questions $(1-4,6,7,10-12,16$, 18,20 ) and was scored on a scale from ' 0 ' (no, or unable to determine) to ' 1 ' (yes) for each question. Scores were summed across questions for each study with a total score of ' 12 ' reflecting the maximum score (highest quality) able to be attained.

\subsection{Study Selection}

After duplicate studies were removed, two reviewers (MM and JW) independently screened all titles and abstracts against inclusion and exclusion criteria of the review. Studies deemed outside the scope of the review were removed. Any conflicts were settled by discussion between the reviewers with a third reviewer consulted for consensus if required. The full-text versions of the remaining studies were then reviewed for eligibility. To be eligible for inclusion, studies were required to: (1) be original research studies; (2) be published in a peer-reviewed journal; (3) have full-text versions available in English language; and (4) have the primary aim of reporting tests used and/or the physical characteristics of adult (i.e. $\geq 18$ years of age) male basketball players. Studies
Table 1 Search strategy used to identify articles

\begin{tabular}{lll}
\hline Search 1 & Search 2 & Search 3 \\
(Male OR men) & (Adult OR senior) & Basketball \\
\hline
\end{tabular}

Search 4

(Fitness testing OR physical characteristics OR Testing OR physical performance OR physical qualities OR physical profile OR anthropometric OR body height OR body weight OR skinfold OR body composition OR body fat OR power OR countermovement jump OR vertical jump OR broad jump OR muscular strength OR muscular endurance OR acceleration OR speed OR sprint OR running OR agility OR change of direction OR fitness OR physical fitness OR aerobic capacity OR repeated-sprint ability OR anaerobic capacity) 
were excluded from the review if they: (1) were systematic or narrative reviews; (2) were meta-analyses; (3) had the primary aim of investigating a nutritional supplement or ergogenic aid; (4) examined referees instead of players; (5) examined wheelchair players; or (6) examined players with a mean age under 18 years or competing in 'junior' competitions. The reference lists of the included studies were then manually reviewed for additional eligible studies. If further studies were identified, they were subjected to the same assessment previously described. Figure 1 outlines the selection process during the screening of studies. Data pertaining to the first aim of this review involved a qualitative synthesis of the available evidence, whereas a quantitative synthesis was used to address the second aim.

\subsection{Data Collection}

Data extraction included study details (authors and publication year), all tests performed to quantify physical characteristics (i.e., height, body mass, wingspan, body fat percentage, muscular power, linear speed, change-of-direction speed, agility, strength, anaerobic capacity, and aerobic capacity), and the outcome variables derived from each test. If the methods of physical testing were not clearly outlined in the study, the tests were not included in the data extraction process. If the authors of the study did not administer the testing protocol as part of the study (e.g., they surveyed coaches for results [38]), the study was not included. If a test included a skill component (e.g., dribbling a basketball) or a series of basketball-specific movements (e.g., sprinting and then jumping), it was not included in the analysis.
Fig. 1 Flow of selection process of eligible studies for qualitative and quantitative synthesis

\section{श्राड MA PRISMA 2009 Flow Diagram}
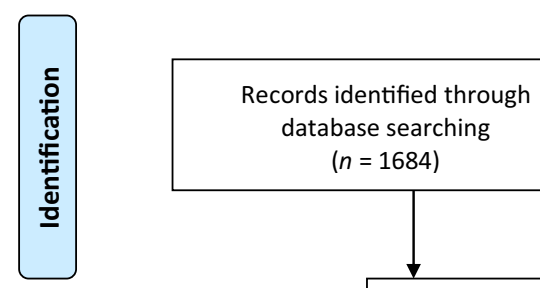
database searching $(n=1684)$

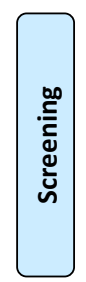

Records after duplicates removed $(n=1309)$
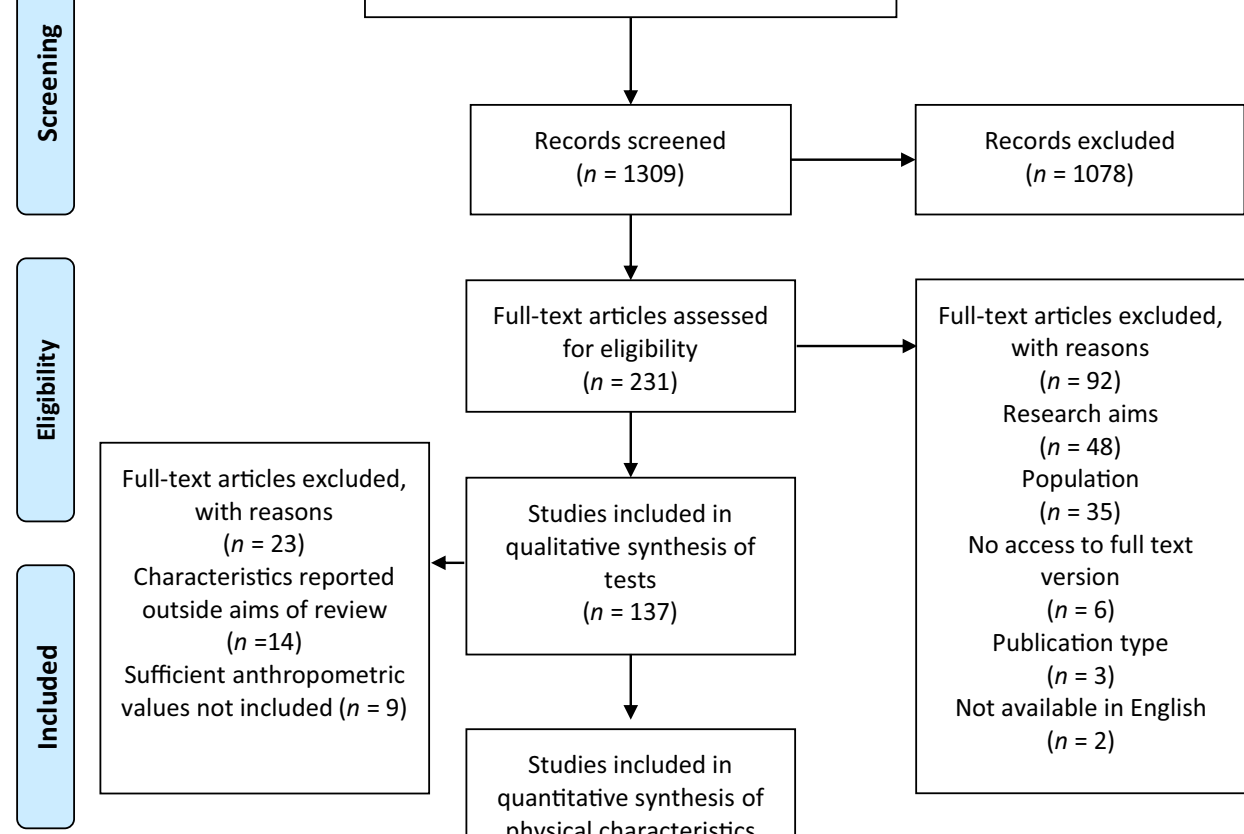

quantitative synthesis of physical characteristics $(n=114)$ 
After the tests were extracted, data relating to playing position and competition level were identified and reported. Competition levels were categorised as either amateur (club, volunteer, or recreational players), collegiate (university or collegiate players), representative (players selected to play in a representative team), semi-professional (some players are contracted or full time) or professional (all players on the team are contracted full-time athletes or competing in a country's highest division or competition). Playing positions were reported as they were identified in the original text of each study. Additionally, outcome variables for anthropometric, muscular power, linear speed, change-of-direction speed, agility, strength, anaerobic capacity, and aerobic capacity tests were retrieved. For tests that had multiple outcome variables, after all data were collated, variables were counted, and the two most frequently used outcome variables were extracted. However, for linear speed and changeof-direction speed tests, only time was extracted because of the variability in other outcome variables reported across studies. Likewise, for assessments of strength, only one repetition maximum (1RM) performances were extracted from studies owing to the variability in other outcome variables. Data were extracted from each study using the raw values provided. In the case of an intervention study (e.g., the implementation of a resistance training programme [39]), baseline measurements were used. Furthermore, if multiple groups were included in a study, the control group was recorded to mitigate the bias of the intervention. To minimise any potential bias or confounding outcomes, studies that did not provide basic player information including age, height, body mass, and competition level were not included in the reporting of physical characteristics (the second aim of this review) but remained in the review to address the first aim. If data were presented using figures and raw data were not clearly available, the authors of the study were contacted to provide the raw values. If no response was received from the authors of a study, means and measures of distribution were extracted from figures in studies using WebPlotDigitizer v4.0 [40], which has been shown as a valid ( $r=0.989$, $p<0.001)$ and reliable $(r=0.997, p<0.001)$ [41] tool for the extraction of raw values from figures. If a study reported variables using units in the Imperial system, they were converted to the Metric system to allow for clear comparison across studies.

To provide greater insight into the physical requirements of professional basketball players competing at the highest level, publicly available NBA Draft Combine data were downloaded from the league's official website [42]. Data representing 100 players (10 players per year over 10 years) were synthesised and used as reference data to describe physical characteristics in this population. The mean, standard deviation, as well as minimum and maximum values for height $(\mathrm{cm})$, body mass $(\mathrm{kg})$, body fat percentage, wingspan (cm), Lane Agility Test time (s), Reactive Shuttle Test time (s), $3 / 4$ court sprint time (s), number of bench press repetitions at $84 \mathrm{~kg}(185 \mathrm{lb})$, vertical jump height $(\mathrm{cm})$, and running vertical jump height $(\mathrm{cm})$ were reported.

\subsection{Categorisation and Presentation of Findings}

The included physical characteristics were chosen given their importance during basketball match-play $[7,9,10$, 20, 43-45]. The three most frequently used tests for each physical characteristic were selected to represent that characteristic. Anthropometric data pertaining to height, wingspan, mass, and body composition were reported. Muscular power was represented indirectly by three bilateral jumping tests: (1) the countermovement jump (CMJ), which represents the ability to use elastic energy that is generated during a preparatory countermovement, without the influence of the arms (i.e., hands placed on hips); (2) the vertical jump (VJ), which involves both a preparatory countermovement and arm swing; and (3) the squat jump (SJ), which represents the concentric only force expressed during a jump. Linear sprint performances over $5 \mathrm{~m}, 10 \mathrm{~m}$, and $20 \mathrm{~m}$ were reported. Change-of-direction speed tests, which differ from assessments of agility because of their predetermined directional requirements and lack of a perceptual decisionmaking component [46], included were the Agility $T$-Test, Lane Agility Test, and Y-Shaped change-of-direction Agility Test. Agility tests, which require players to change their movement in response to a stimulus [46, 47], included the Reactive Y-Change-of-Direction Test, the Reactive Changeof-Direction Test, and the Reactive Agility Test. Strength was categorised as lower-body and upper-body strength, using the back squat and bench press, respectively. Only two tests were provided for strength characteristics because of the variability in the remaining tests across studies. Anaerobic capacity was reported using the Wingate Anaerobic Cycle Test (WAnT), full court shuttle run, and the Runningbased Anaerobic Speed Test (RAST). Aerobic capacity was reported using tests that assessed maximal oxygen uptake $\left(\mathrm{VO}_{2 \max }\right)$ or distance covered during a maximal running test. The three tests predominantly used to assess aerobic capacity were the Yo-Yo Intermittent Recovery Test Level 1 (Yo-Yo IRL1), Multi-Stage Fitness Test (MSFT), and incremental treadmill tests.

\section{Results}

\subsection{Identification and Selection of Articles}

The search of databases identified 1684 studies. A total of 375 duplicates were removed, resulting in 1309 studies to be screened by title and abstract. After screening, 231 studies 
were eligible for full-text review with a further 59 eligible studies identified in the reference lists during the full-text screening. After full-text screening a total of 137 studies were identified including tests and outcome variables while 114 studies reported physical characteristics in adult male basketball players. Inter-reviewer reliability was calculated using Cohen's Kappa statistic $(K=0.85)$.

\subsection{Assessment of Reporting Quality}

Reporting quality scores ranged from 6 to 11 for the 12 items assessed in the modified Downs and Black checklist, with a mean score of $9.47 \pm 0.83$ across the included studies (Table 1 of the ESM).

\subsection{Data Collection Methods}

The tests and outcome variables used to assess the physical characteristics of adult male basketball players across all competition levels are displayed in Tables 3-10 of the ESM. Tests were categorised based on the characteristic they assessed; body composition, muscular power, linear speed, change-of-direction speed, agility, strength, anaerobic capacity, and aerobic capacity.

\subsection{Overview of Included Studies and Tests}

A total of 134 tests and 394 outcome variables assessing physical characteristics in adult male basketball players across all competition levels were included in this review. Table 2 summarises tests used across included studies to represent each physical characteristic.

\subsection{NBA Draft Combine Data Extraction}

Data pertaining to the physical characteristics of 100 players drafted into the NBA between the 2011-12 and 2020-21 seasons are presented in Table 3 . The mean draft pick number of the players who participated in the NBA Draft Combine increased yearly from $9 \pm 4$ in $2011-12$ to $35 \pm 10$ in 2020-21. Performance during the Reactive Shuttle Run was only available from the 2013-14 season. Bench press performance was not reported in the 2014-15, 2016-17, and 2020-21 seasons.

\subsection{Anthropometric Characteristics}

Height and body mass were reported in 116 (85\%) of the 137 studies included in this review. Anthropometric data (i.e., height, body mass and body fat percentage) were reported according to playing position (Figs. 2, 3,4) or as a mean across the entire team (Table 2 of the ESM).

Table 2 Tests selected to report the physical characteristics of adult male basketball players in this review

\begin{tabular}{|c|c|c|c|}
\hline Category & Test & Outcome variable & Citations \\
\hline \multirow[t]{3}{*}{ Muscular power } & Countermovement jump & Peak power and jump height & 46 \\
\hline & Squat jump & Peak power and jump height & 20 \\
\hline & Vertical jump & Peak power and jump height & 33 \\
\hline \multirow[t]{3}{*}{ Linear speed } & 5-m sprint & Time & 10 \\
\hline & 10-m sprint & Time & 18 \\
\hline & 20-m sprint & Time & 20 \\
\hline \multirow[t]{3}{*}{ COD speed } & Agility $T$-Test & Time & 20 \\
\hline & Y-Shaped COD & Time & 7 \\
\hline & Lane Agility Test & Time & 4 \\
\hline Agility & Y-Shaped Agility Tests & Time, response time, and decision-making time & 7 \\
\hline \multirow[t]{2}{*}{ Strength } & Bench press & $1 \mathrm{RM}$ & 17 \\
\hline & Back squat & $1 \mathrm{RM}$ & 7 \\
\hline \multirow[t]{3}{*}{ Aerobic capacity } & Yo-Yo IRL1 & Estimated $\mathrm{VO}_{2 \max }$ and distance & 12 \\
\hline & Multi-Stage Fitness Test & Estimated $\mathrm{VO}_{2 \max }$ and number of shuttles & 10 \\
\hline & Incremental Treadmill Tests & $\mathrm{VO}_{2 \max }$ & 21 \\
\hline \multirow[t]{3}{*}{ Anaerobic capacity } & Full Court Shuttle Run & Time & 5 \\
\hline & RAST & Peak power, mean power, and fatigue index & 6 \\
\hline & Wingate Anaerobic Cycle Test & Peak power, mean power, and fatigue index & 8 \\
\hline
\end{tabular}

$1 R M$ one repetition maximum, $C O D$ change-of-direction, $R A S T$ Running-Based Anaerobic Speed Test, $V O_{2 m a x}$ maximum oxygen uptake, $Y o-Y o$ IRL1 Yo-Yo Intermittent Recovery Test Level 1 
Table 3 Summary of National Basketball Association Draft Combine performance over the previous 10 years

\begin{tabular}{lcll}
\hline Test & Mean \pm SD & Minimum & Maximum \\
\hline Height $(\mathrm{cm})$ & $197.7 \pm 7.6$ & 181.6 & 212.7 \\
Wingspan (cm) & $210 \pm 8.6$ & 187.3 & 231.8 \\
Body mass (kg) & $97.2 \pm 10.5$ & 77.1 & 126.4 \\
Body fat \% & $6.7 \pm 1.9$ & 3.2 & 13.6 \\
Lane Agility Test (s) & $11.1 \pm 0.4$ & 10.3 & 12.2 \\
Reactive Shuttle Run (s) & $3.0 \pm 0.2$ & 2.3 & 3.7 \\
3/4 Court sprint (s) & $3.3 \pm 0.1$ & 3.6 & 3.0 \\
Bench press & $8.8 \pm 4.7$ & 0.0 & 20.0 \\
Vertical jump (cm) & $77.4 \pm 7.7$ & 62.2 & 96.5 \\
Running vertical jump & $92.4 \pm 7.9$ & 74.9 & 110.5 \\
$\quad$ (cm) & & & \\
Draft pick & $16.6 \pm 10.1$ & 2 & 50 \\
\hline
\end{tabular}

$S D$ standard deviation. Bench press = number of completed bench press repetitions at $84 \mathrm{~kg}(185 \mathrm{lb})$

Mean height ranged between 177 and $214 \mathrm{~cm}$ across studies. The mean height of professional $(183-202 \mathrm{~cm})$ and collegiate $(177-201 \mathrm{~cm})$ players had similar ranges. Additionally, semi-professional $(182-198 \mathrm{~cm})$ and representative $(182-197 \mathrm{~cm})$ players had mean heights that were also comparable. Finally, the shortest players were observed at the amateur level with mean height range from 180 to $195 \mathrm{~cm}$. When mean height was reported according to playing position, guards (183-193 cm [Fig. 2]) were consistently reported as being shorter than forwards (190-202 cm [Fig. 3]), with centres observed as the tallest players (198-214 cm [Fig. 4]). Positional mean heights at the professional level followed the same trend (guards: $183-193 \mathrm{~cm}$, forwards: 190-201 cm, and centres 198-214 cm). Furthermore, three studies suggested the same positional trend was present in the mean height of semi-professional players (guards: $183-187 \mathrm{~cm} \mathrm{[4,} \mathrm{48,} \mathrm{49],} \mathrm{forwards:} 194 \pm 5 \mathrm{~cm}$ [48, 49], forwards and centres: $194 \pm 7 \mathrm{~cm}[4]$, and centres: $198 \pm 5 \mathrm{~cm}[48,49])$. Only two studies (guards: $187 \pm 7 \mathrm{~cm}$ [50], forwards: $202 \pm 4 \mathrm{~cm} \mathrm{[50],} \mathrm{backcourt:} 188 \pm 6 \mathrm{~cm} \mathrm{[3],}$ and frontcourt: $201 \pm 6 \mathrm{~cm} \mathrm{[3])} \mathrm{reported} \mathrm{collegiate} \mathrm{player}$ height according to playing position, while no studies reported height in specific playing positions at the representative or amateur levels.

Mean body mass reported across studies ranged between 68 and $111 \mathrm{~kg}$ (Figs. 2, 3, 4 and Table 2 of the ESM). The range in mean body mass of players competing at various competition levels were: professional: $76-105 \mathrm{~kg}$; semiprofessional: $74-90 \mathrm{~kg}$; representative: $76-100 \mathrm{~kg}$; collegiate: $69-101 \mathrm{~kg}$; and amateur: $68-94 \mathrm{~kg}$. Observing mean body mass by playing position revealed guards $(77-90 \mathrm{~kg}$ [Fig. 2]), were typically lightest, with forwards being heavier than guards (82-105 kg [Fig. 3]), and centres being the heaviest (93-111 kg [Fig. 4]). Professional guards had mean body masses between 77 and $90 \mathrm{~kg}$, professional forwards between 82 and $100 \mathrm{~kg}$, and centres between 96 and $111 \mathrm{~kg}$. Three studies reported body mass by playing position at the semi-professional level (guards: $78.1 \pm 6 \mathrm{~kg}$ [48, 49], $85.5 \pm 12.3 \mathrm{~kg}$ [4], forwards: $89.5 \pm 7.9 \mathrm{~kg}[48,49]$, forwards and centres: $109.4 \pm 8.8 \mathrm{~kg}$ [4], and centres: $92.6 \pm 8.2 \mathrm{~kg}$ $[48,49])$. Only two studies reported body mass by playing position at the collegiate level (guards: $85.2 \pm 7.4 \mathrm{~kg}$ [50], forwards: $105.3 \pm 8 \mathrm{~kg}$ [50], backcourt: $83.3 \pm 8.1 \mathrm{~kg}$ [3], and frontcourt: $108.1 \pm 9.9 \mathrm{~kg}$ [3]). No studies reported body mass by playing position at the representative or amateur levels.

Wingspan was reported in three studies [3, 51, 52] with a mean value range from 194 to $207 \mathrm{~cm}$. One study [51] observed a wingspan of $200 \pm 10 \mathrm{~cm}$ in a team of collegiate NCAA Division 2 players. A second study [3] reported a wingspan of $199 \pm 10 \mathrm{~cm}$ across the team, with data also provided according to playing position (backcourt: $194 \pm 10 \mathrm{~cm}$ and frontcourt: $205 \pm 3 \mathrm{~cm}$ ) in collegiate NCAA Division 1 players. Finally, the wingspans of guards $(190 \pm 5 \mathrm{~cm})$, forwards $(197 \pm 6 \mathrm{~cm})$, centres $(207 \pm 8 \mathrm{~cm})$ and the entire team $(198 \pm 9 \mathrm{~cm})$ were observed in players competing professionally in Poland [52].

Body composition was assessed in 68 (50\%) of the 137 studies included in this review, with 14 different types of tests and nine outcome variables used (Table 3 of the ESM). Data pertaining to body composition by playing position are reported in Fig. 2 (guards), Fig. 3 (forwards) and Fig. 4 (centres), and mean team measurements are provided in Table 2 of the ESM. The most frequently implemented test and outcome variable used across studies were the sum of skinfolds at three sites (chest, abdomen, and thigh [16, 53-58], triceps, pectoral, and subscapular [59], and triceps, abdomen, and thigh $[4,21,60])$ and body fat percentage, respectively (Table 3 of the ESM).

Mean body composition ranged between 7 and 24\% body fat across studies (Figs. 2, 3, 4 and Table 2 of the ESM). Mean body fat percentage across competition levels revealed professional players varied between 7 and $20 \%$, while semiprofessional (9-16\%), collegiate (10-14\%), and representative (8-14\%) levels exhibited similar ranges in body fat percentage. Amateur players possessed varied mean body composition measurements of between 10 and $24 \%$ body fat. When mean body composition was reported according to playing position, guards (7-20\% [Fig. 2]), forwards (8-17\% [Fig. 3]), and centres (7-21\% [Fig. 4]) demonstrated similar variance in body fat percentage. Professional guards (7-20\%), forwards $(8-17 \%)$, and centres (7-21\%) also possessed similar levels of body fat. Semi-professional guard or backcourt (9-13\%) and forward or frontcourt (11-17\%) body fat percentages were reported in three studies $[4,48$, 49 ], whereas centres $(11.7 \pm 4.1 \%)$ were only reported in two studies [48, 49]. Body fat percentages were reported 


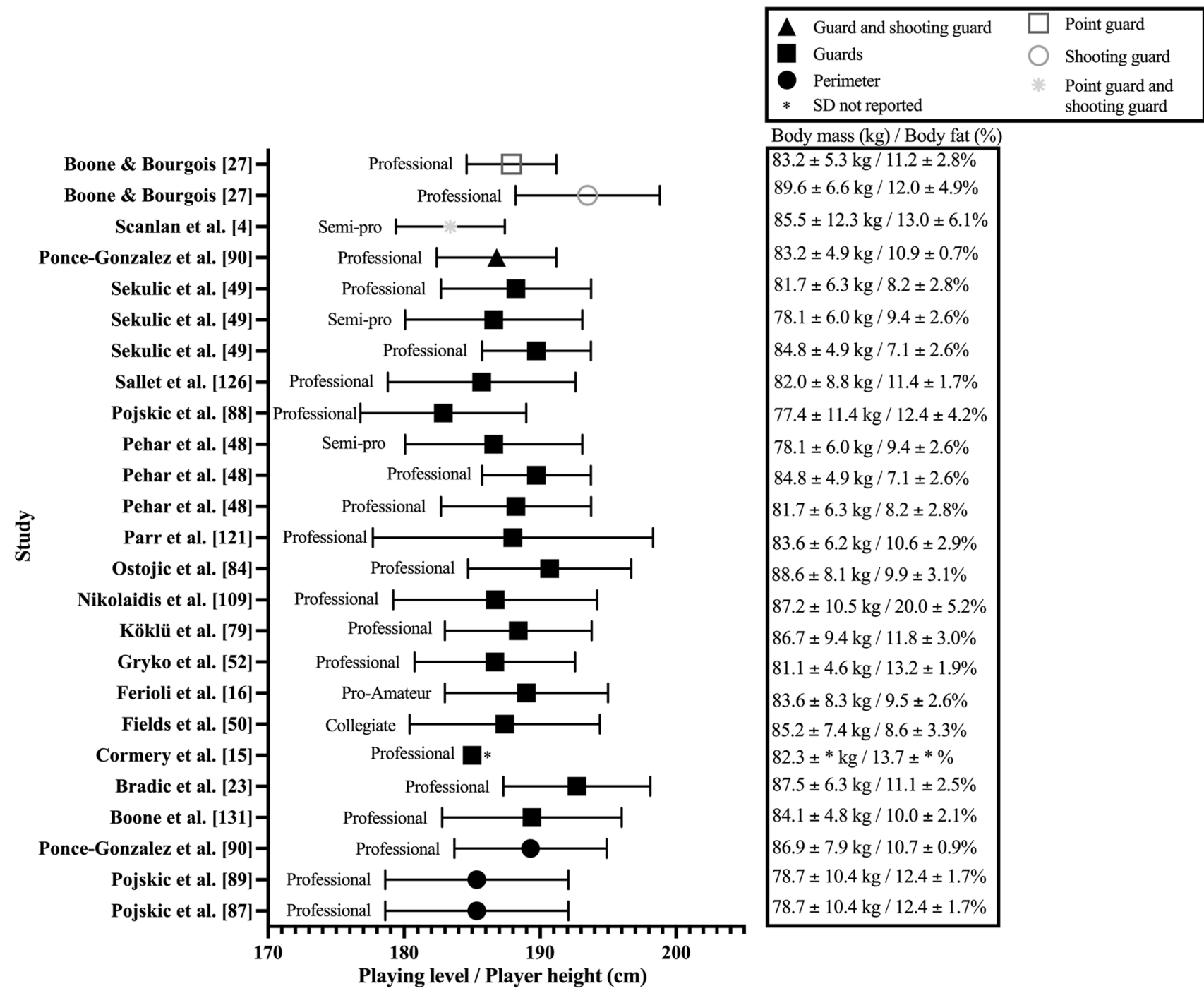

Fig. 2 Height, mass, and body fat percentage of the guard playing position in adult male basketball players

for guard and forward positions at the collegiate level in one study [50] (Figs. 2, 3). No studies reported body fat percentage relative to playing position at the representative or amateur playing levels.

\subsection{Muscular Power}

Muscular power was assessed predominantly using jump tests, with $80(58 \%)$ of the 137 studies in this review employing 18 different jump tests (Table 4 of the ESM). The three most frequent jump tests adopted across studies were the CMJ (43 studies, $54 \%$ of studies assessing muscular power) $[14,16,27,48,51,54,55,58,61-7577-96]$, VJ (27 studies, $34 \%$ of studies assessing muscular power) [3, 14, 28, $39,56,59,62,66,82,90-92,96-100,102-111]$, and SJ (15 studies, $18 \%$ of studies assessing muscular power) $[27,58$, $61,63,67,72,77-79,81,89,90,93,95,96]$. Additional jump tests used across studies are reported in Table 4 of the ESM. The most commonly reported outcome variables were jump height and peak power (Table 4 of the ESM). Two throwing tests were also used in studies to assess muscular power including a seated basketball throw [68] with speed $(\mathrm{km} /$ hour $)$ of the throw taken as the outcome variable, and a seated medicine ball throw $(1 \mathrm{~kg}[87,89]$ and unknown mass [56]) with horizontal displacement (m) of the ball used as the outcome variable. Additionally, muscular power variables were also recorded during tests predominantly implemented to assess strength. These tests are reported in Sect. 3.2.7 and include bench press and squat exercises (Table 8 of the ESM).

Jump performance variables reported across studies during the $\mathrm{CMJ}, \mathrm{VJ}$, and $\mathrm{SJ}$ are provided in Tables 4,5 , and 6. Mean CMJ height ranged between 34 and $77 \mathrm{~cm}$, while mean peak power outputs ranged between 2441 and $6647 \mathrm{~W}$ 


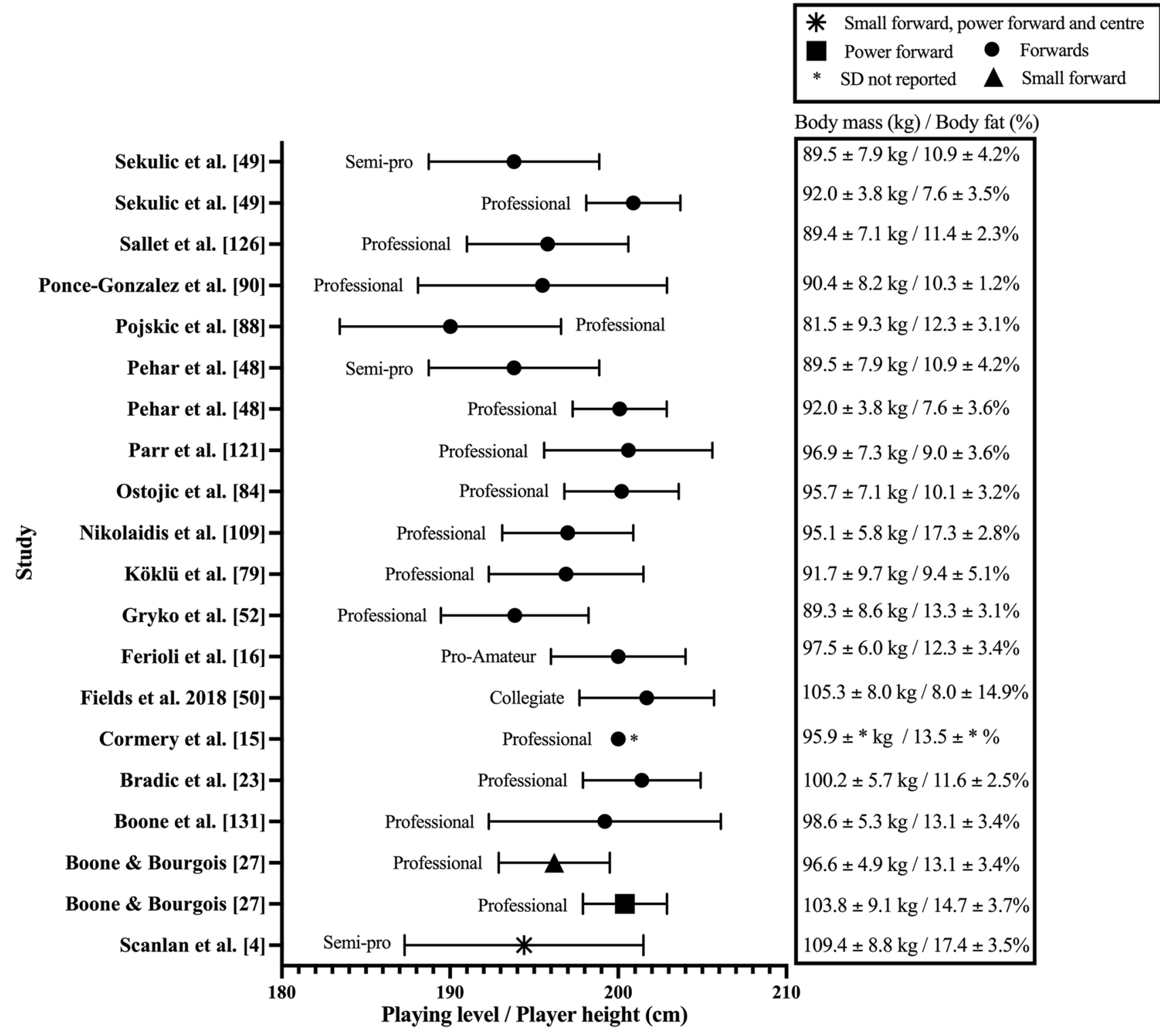

Fig. 3 Height, mass, and body fat percentage of the forward playing position in adult male basketball players

(Table 4). In professional players, mean CMJ height and mean CMJ peak power ranged between 36 and $63 \mathrm{~cm}$ and between 3874 and $5468 \mathrm{~W}$, respectively. Mean CMJ height $(34-50 \mathrm{~cm})$ and mean CMJ peak power (2441-5078 W) were lower in semi-professional players than professional players, while collegiate players had the greatest mean jump height $(36-77 \mathrm{~cm})$ and peak power output (4736-6647 W). Countermovement jump height was only reported in one study [65] at the representative level with CMJ peak power not reported. Countermovement jump height and CMJ peak power were reported at the amateur level in one study [16] (Table 4). Countermovement jump performance was only reported according to playing position at the professional level $[14,27,48,79,84,88,90]$, the collegiate level [75] and as a combined group of players from amateur to professional levels [16]. Similar mean CMJ heights were evident between positions in professional players (guards: $38-60 \mathrm{~cm}$, forwards: $36-58 \mathrm{~cm}$, and centres: $36-57 \mathrm{~cm}$ ), while mean absolute peak power was lowest in guards (3874-4510 W), then forwards (3930-5221 W), and greatest in centres (4536-5353 W).

Mean jump height measured during the $\mathrm{VJ}$ was the greatest across all jump tests, with a range from 39 to 83 $\mathrm{cm}$, while mean VJ peak power ranged between 2121 and $6701 \mathrm{~W}$ (Table 5). Professional players recorded mean VJ heights between 39 and $69 \mathrm{~cm}$ and mean VJ peak power outputs of 2215-6701 W during the VJ. Semi-professional players reached mean VJ heights between 41 and $65 \mathrm{~cm}$. 


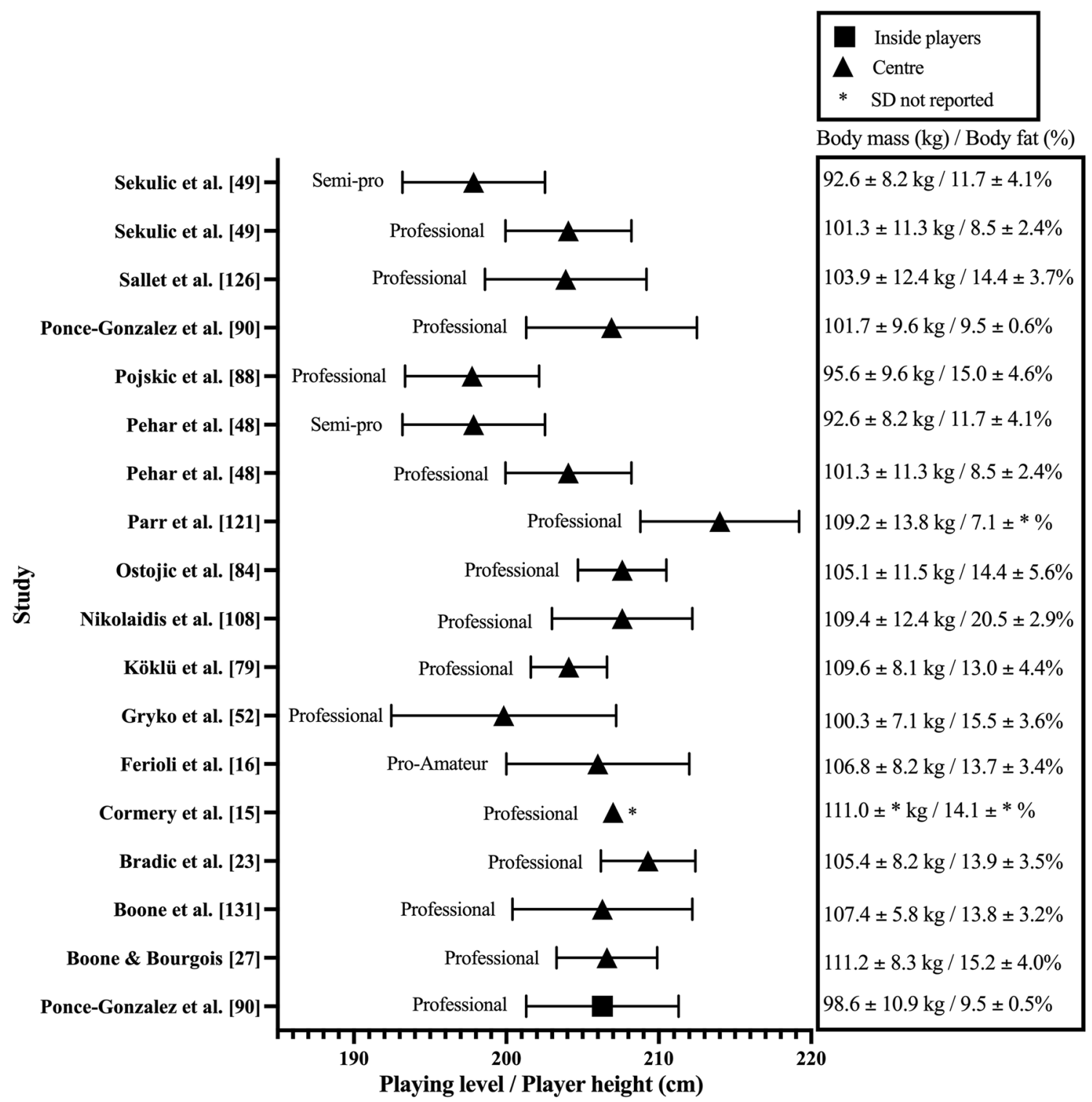

Fig. 4 Height, mass, and body fat percentage of the centre playing position in adult male basketball players

However, only two studies $[66,107]$ reported peak power output (2121-3591 W) for semi-professional players during the VJ. Collegiate players recorded mean VJ heights between 44 and $83 \mathrm{~cm}$, while no studies reported peak power output. No studies reported VJ performance in representative players. Two studies $[97,98]$ reported VJ height in amateur players $(41-52 \mathrm{~cm})$, while no studies reported peak power output in amateur players. Vertical jump height relative to playing position (guards: 44-65 cm, forwards: $44-64 \mathrm{~cm}$, and centres: $39-63 \mathrm{~cm}$ ) was only reported in professional players across three studies [14, 90, 109], one of which measured peak power [90] (Table 5). Positional VJ performance was only reported in one study in semi-professional [28] and collegiate [3] players (Table 5). No studies reported positional VJ performance in representative or amateur players.

Mean SJ height ranged between 27 and $58 \mathrm{~cm}$, while mean SJ peak power outputs were only reported in professional players and ranged between 3639 and $5149 \mathrm{~W}$ (Table 6). Professional players reached mean SJ jump heights between 29 and $50 \mathrm{~cm}$. Only one study [58] reported SJ height in semi-professional players, while peak power was not reported. Squat jump height in collegiate players was only reported in one study [95], while no studies reported SJ peak power output in collegiate players. No studies reported SJ height or peak power in representative or amateur players. Mean SJ height [27, 79, 90] (guards: $30-41 \mathrm{~cm}$, forwards: 29-40 cm, and centres: $33-36 \mathrm{~cm}$ ) and peak power [27, 90] 
Table 4 Jump height and peak power variables reported during the countermovement jump in adult male basketball players

\begin{tabular}{|c|c|c|c|c|c|}
\hline Study & Playing position & Competition level & Category & Jump height $(\mathrm{cm})$ & Peak power $(\mathrm{W})$ \\
\hline Alemdaroglu [61] & All & Turkish D1 & Professional & $34.9 \pm 3.8$ & \\
\hline Annino et al. [62] & All & Italian National Federal League L2 & Professional & $38.9 \pm 3.6$ & \\
\hline Aoki et al. [63] & All & Brazilian National League & Professional & $38.1 \pm 2.8$ & \\
\hline $\begin{array}{l}\text { Barrera-Domínguez et al. } \\
\text { [64] }\end{array}$ & All & Spanish National Division & Professional & $35.6 \pm 4.8$ & \\
\hline Ben Abdelkrim et al. [65] & $\begin{array}{l}\text { All (U20) } \\
\text { All }\end{array}$ & $\begin{array}{l}\text { Tunisian National Team } \\
\text { Tunisian National Team }\end{array}$ & $\begin{array}{l}\text { Representative } \\
\text { Professional }\end{array}$ & $\begin{array}{l}49.1 \pm 5.9 \\
49.7 \pm 5.8\end{array}$ & $\begin{array}{l}4656 \pm 81 \\
4665 \pm 116\end{array}$ \\
\hline Boone and Bourgois [27] & $\begin{array}{l}\text { Point guard } \\
\text { Shooting guard } \\
\text { Small forward } \\
\text { Power forward } \\
\text { Centre }\end{array}$ & $\begin{array}{l}\text { Belgian D1 } \\
\text { Belgian D1 } \\
\text { Belgian D1 } \\
\text { Belgian D1 } \\
\text { Belgian D1 }\end{array}$ & $\begin{array}{l}\text { Professional } \\
\text { Professional } \\
\text { Professional } \\
\text { Professional } \\
\text { Professional }\end{array}$ & $\begin{array}{l}42.7 \pm 3.8 \\
41.3 \pm 3.2 \\
42.5 \pm 3.8 \\
42.4 \pm 3.7 \\
36.2 \pm 4.1\end{array}$ & $\begin{array}{l}4306 \pm 373 \\
4510 \pm 322 \\
4901 \pm 387 \\
5221 \pm 364 \\
5180 \pm 451\end{array}$ \\
\hline Buśko et al. [66] & All & Warsaw Sports Club Polonia D2 & $\begin{array}{l}\text { Semi-profes- } \\
\text { sional }\end{array}$ & $41.9 \pm 4.0$ & $2441 \pm 440$ \\
\hline Chaouachi et al. [67] & All & Tunisian National Team & Professional & $61.9 \pm 6.2$ & \\
\hline Chen et al. [68] & All & Collegiate D1 & Collegiate & $45.6 \pm 4.0$ & \\
\hline Ciacci and Bartolomei [96] & $\begin{array}{l}\text { All } \\
\text { All }\end{array}$ & $\begin{array}{l}\text { National Level } \\
\text { National Level }\end{array}$ & $\begin{array}{l}\text { Professional } \\
\text { Professional }\end{array}$ & $\begin{array}{l}39.2 \pm 5.7 \\
41.9 \pm 5.2\end{array}$ & \\
\hline Dawes and Spiteri [51] & All & NCAA D2 & Collegiate & $76.9 \pm 7.5$ & \\
\hline Dello Iacono et al. [69] & All & Professional Basketball Club UK & Professional & $60.4 \pm N R$ & \\
\hline Ferioli et al. [54] & $\begin{array}{l}\text { All } \\
\text { All }\end{array}$ & $\begin{array}{l}\text { Italian Serie A and Serie A2 } \\
\text { Italian Serie B }\end{array}$ & $\begin{array}{l}\text { Professional } \\
\text { Semi-profes- } \\
\quad \text { sional }\end{array}$ & $\begin{array}{l}50.3 \pm 5.4 \\
49.4 \pm 5.4\end{array}$ & $\begin{array}{l}5153 \pm 593 \\
4405 \pm 667\end{array}$ \\
\hline Ferioli et al. [16] & $\begin{array}{l}\text { All } \\
\text { All } \\
\text { All } \\
\text { All } \\
\text { Guard } \\
\text { Forward } \\
\text { Centre }\end{array}$ & $\begin{array}{l}\text { Italian Serie A } \\
\text { Italian Serie A2 } \\
\text { Italian Serie B } \\
\text { Italian Serie D } \\
\text { Italian Serie A-D } \\
\text { Italian Serie A-D } \\
\text { Italian Serie A-D }\end{array}$ & $\begin{array}{l}\text { Professional } \\
\text { Professional } \\
\text { Semi-profes- } \\
\text { sional } \\
\text { Amateur } \\
\text { Amateur - Pro } \\
\text { Amateur - Pro } \\
\text { Amateur - Pro }\end{array}$ & $\begin{array}{l}47.8 \pm 5.7 \\
49.2 \pm 4.9 \\
48.0 \pm 6.1 \\
51.8 \pm 4.1 \\
49.2 \pm 4.9 \\
48.6 \pm 6.0 \\
45.8 \pm 6.0\end{array}$ & $\begin{array}{l}5468 \pm 820 \\
5177 \pm 629 \\
4685 \pm 723 \\
4800 \pm 536 \\
4785 \pm 678 \\
5436 \pm 738 \\
5560 \pm 682\end{array}$ \\
\hline Ferioli et al. [55] & $\begin{array}{l}\text { All } \\
\text { All } \\
\text { All }\end{array}$ & $\begin{array}{l}\text { Italian Serie A } \\
\text { Italian Serie A2 } \\
\text { Italian Serie B }\end{array}$ & $\begin{array}{l}\text { Professional } \\
\text { Professional } \\
\text { Semi-profes- } \\
\quad \text { sional }\end{array}$ & $\begin{array}{l}46.9 \pm 4.4 \\
50.9 \pm 5.6 \\
50.1 \pm 4.8\end{array}$ & $\begin{array}{l}5282 \pm 582 \\
5182 \pm 745 \\
4691 \pm 624\end{array}$ \\
\hline Freitas et al. [70] & All & Spanish Liga EBA D4 & $\begin{array}{l}\text { Semi-profes- } \\
\text { sional }\end{array}$ & $35.0 \pm 7.0$ & $5078 \pm 437$ \\
\hline Freitas et al. [71] & All & Spanish Liga EBA D4 & $\begin{array}{l}\text { Semi-profes- } \\
\text { sional }\end{array}$ & $36.5 \pm 7.2$ & $4699 \pm 781$ \\
\hline Gomes et al. [72] & All & PSCB & Professional & $39.3 \pm 5.6$ & \\
\hline Heishman et al. [74] & All & NCAA D1 & Collegiate & $58.3 \pm 1.4$ & $6374 \pm 165$ \\
\hline Heishman et al. [73] & All & NCAA D1 & Collegiate & $62.8 \pm 1.5$ & $6647 \pm 171$ \\
\hline Heishman et al. [75] & $\begin{array}{l}\text { All } \\
\text { Guards } \\
\text { Frontcourt }\end{array}$ & $\begin{array}{l}\text { NCAA D1 } \\
\text { NCAA D1 } \\
\text { NCAA D1 }\end{array}$ & $\begin{array}{l}\text { Collegiate } \\
\text { Collegiate } \\
\text { Collegiate }\end{array}$ & $\begin{array}{l}38.7 \pm 6.4 \\
42.6 \pm 0.4 \\
34.6 \pm 0.4\end{array}$ & \\
\hline Jallai et al. [77] & All & Estonian $1^{\text {st }}$ League & Professional & $43.2 \pm 2.3$ & \\
\hline Khlifa et al. [78] & All & Tunisian D1 & Professional & $45.2 \pm 1.3^{\#}$ & \\
\hline Köklü et al. [79] & $\begin{array}{l}\text { All } \\
\text { All } \\
\text { All } \\
\text { Guard } \\
\text { Forward } \\
\text { Centre }\end{array}$ & $\begin{array}{l}\text { Turkish D1 and D2 } \\
\text { Turkish D1 } \\
\text { Turkish D2 } \\
\text { Turkish D1 and D2 } \\
\text { Turkish D1 and D2 } \\
\text { Turkish D1 and D2 }\end{array}$ & $\begin{array}{l}\text { Professional } \\
\text { Professional } \\
\text { Professional } \\
\text { Professional } \\
\text { Professional } \\
\text { Professional }\end{array}$ & $\begin{array}{l}38.3 \pm 5.3 \\
40.6 \pm 4.7 \\
36.0 \pm 5.0 \\
38.2 \pm 5.8 \\
40.1 \pm 5.1 \\
36.6 \pm 4.7\end{array}$ & \\
\hline Laplaud et al. [80] & All & Professional & Professional & $63.0 \pm 9.0$ & \\
\hline Maffiuletti et al. [81] & All & French Basketball Federation D2 & Professional & $51.0 \pm 1.3$ & \\
\hline
\end{tabular}


Table 4 (continued)

\begin{tabular}{|c|c|c|c|c|c|}
\hline Study & Playing position & Competition level & Category & Jump height (cm) & Peak power $(\mathrm{W})$ \\
\hline Maggioni et al. [58] & All & Volunteer Players & $\begin{array}{l}\text { Semi-profes- } \\
\text { sional }\end{array}$ & $32.0 \pm 4.9$ & \\
\hline Mandic et al. [82] & All & National League Serbia & Professional & $36.9 \pm 3.7$ & \\
\hline Miura et al. [83] & All & National Collegiate Japan & Collegiate & $50.5 \pm 5.4$ & \\
\hline Ostojic et al. [84] & $\begin{array}{l}\text { All } \\
\text { Guard } \\
\text { Forward } \\
\text { Centre }\end{array}$ & $\begin{array}{l}\text { First National League Serbia } \\
\text { First National League Serbia } \\
\text { First National League Serbia } \\
\text { First National League Serbia }\end{array}$ & $\begin{array}{l}\text { Professional } \\
\text { Professional } \\
\text { Professional } \\
\text { Professional }\end{array}$ & $\begin{array}{l}57.4 \pm 7.7 \\
59.7 \pm 9.6 \\
57.8 \pm 6.5 \\
54.6 \pm 6.9\end{array}$ & \\
\hline Pehar et al. [48] & $\begin{array}{l}\text { All } \\
\text { All } \\
\text { Guard } \\
\text { Forward } \\
\text { Centre } \\
\text { Guard } \\
\text { Guard } \\
\text { Forward } \\
\text { Forward } \\
\text { Centre } \\
\text { Centre }\end{array}$ & $\begin{array}{l}\text { Bosnia and Herzegovina D1 } \\
\text { Bosnia and Herzegovina D2 } \\
\text { Bosnia and Herzegovina D1 and D2 } \\
\text { Bosnia and Herzegovina D1 and D2 } \\
\text { Bosnia and Herzegovina D1 and D2 } \\
\text { Bosnia and Herzegovina D1 } \\
\text { Bosnia and Herzegovina D2 } \\
\text { Bosnia and Herzegovina D1 } \\
\text { Bosnia and Herzegovina D2 } \\
\text { Bosnia and Herzegovina D1 } \\
\text { Bosnia and Herzegovina D2 }\end{array}$ & $\begin{array}{l}\text { Professional } \\
\text { Professional } \\
\text { Professional } \\
\text { Professional } \\
\text { Professional } \\
\text { Professional } \\
\text { Professional } \\
\text { Professional } \\
\text { Professional } \\
\text { Professional } \\
\text { Professional }\end{array}$ & $\begin{array}{l}45.5 \pm 5.6 \\
45.3 \pm 6.1 \\
46.4 \pm 6.0 \\
45.5 \pm 5.5 \\
43.9 \pm 5.5 \\
46.9 \pm 5.4 \\
46.0 \pm 6.6 \\
46.5 \pm 5.3 \\
44.5 \pm 5.9 \\
43.7 \pm 5.6 \\
44.5 \pm 5.6\end{array}$ & \\
\hline Pehar et al. [85] & All & Bosnia and Herzegovina D1 & Professional & $45.6 \pm 5.5$ & \\
\hline Pliauga et al. [86] & All & $\begin{array}{l}\text { Lithuanian National Basketball } \\
\text { League }\end{array}$ & Collegiate & $47.8 \pm 3.0$ & \\
\hline \multirow[t]{3}{*}{ Pojskić et al. [88] } & Guard & Bosnian Premier League & Professional & $40.4 \pm 5.0$ & $3874 \pm 639$ \\
\hline & Forward & Bosnian Premier League & Professional & $37.6 \pm 6.8$ & $3930 \pm 604$ \\
\hline & Centre & Bosnian Premier League & Professional & $36.0 \pm 3.8$ & $4536 \pm 458$ \\
\hline Pojskić et al. [87] & Perimeter & Bosnian Premier League & Professional & $38.5 \pm 3.5$ & \\
\hline Pojskić et al. [89] & Perimeter & Bosnian and Herzegovina D1 & Professional & $38.5 \pm 3.5$ & \\
\hline Ponce-González et al. [90] & $\begin{array}{l}\text { All } \\
\text { Guard } \\
\text { Forward } \\
\text { Centre } \\
\text { Perimeter } \\
\text { Inside }\end{array}$ & $\begin{array}{l}\text { Spanish Pro Liga ACB } \\
\text { Spanish Pro Liga ACB } \\
\text { Spanish Pro Liga ACB } \\
\text { Spanish Pro Liga ACB } \\
\text { Spanish Pro Liga ACB } \\
\text { Spanish Pro Liga ACB }\end{array}$ & $\begin{array}{l}\text { Professional } \\
\text { Professional } \\
\text { Professional } \\
\text { Professional } \\
\text { Professional } \\
\text { Professional }\end{array}$ & $\begin{array}{l}36.8 \pm 4.1 \\
37.7 \pm 3.8 \\
35.6 \pm 4.6 \\
37.2 \pm 4.9 \\
37.4 \pm 3.8 \\
36.1 \pm 4.9\end{array}$ & $\begin{array}{l}4707 \pm 676 \\
4159 \pm 218 \\
4607 \pm 606 \\
5353 \pm 538 \\
4399 \pm 521 \\
5137 \pm 672\end{array}$ \\
\hline \multirow[t]{4}{*}{ Puente et al. [14] } & All & $\begin{array}{l}\text { National Spanish Basketball Federa- } \\
\text { tion }\end{array}$ & Professional & $58 \pm 4$ & \\
\hline & Guard & $\begin{array}{l}\text { National Spanish Basketball Federa- } \\
\text { tion }\end{array}$ & $\begin{array}{l}\text { Professional } \\
\text { Professional }\end{array}$ & $\begin{array}{l}58 \pm 3 \\
58 \pm 5\end{array}$ & \\
\hline & Forward & $\begin{array}{l}\text { National Spanish Basketball Federa- } \\
\text { tion }\end{array}$ & & & \\
\hline & Centre & $\begin{array}{l}\text { National Spanish Basketball Federa- } \\
\text { tion }\end{array}$ & Professional & $57 \pm 2$ & \\
\hline $\begin{array}{l}\text { Rodriguez-Rosell et al. } \\
\text { [91] }\end{array}$ & All & Spanish Liga EBA D4 & $\begin{array}{l}\text { Semi-profes- } \\
\text { sional }\end{array}$ & $34.8 \pm 5.8$ & \\
\hline Schiltz et al. [92] & All & European Cup D1 & Professional & $40.5 \pm 5.7$ & \\
\hline Shalfawi et al. [93] & All & Professional level Norway & Professional & $52.0 \pm 7.5$ & $5167 \pm 419$ \\
\hline Stojanovic et al. [94] & All & Serbian Professional League & Professional & $39.8 \pm 5.1$ & \\
\hline Xie et al. [95] & $\begin{array}{l}\text { All } \\
\text { All }\end{array}$ & $\begin{array}{l}\text { NCAA D1 university team } \\
\text { NCAA D1 club team }\end{array}$ & $\begin{array}{l}\text { Collegiate } \\
\text { Collegiate }\end{array}$ & $\begin{array}{l}71.9 \pm 9.1 \\
72.7 \pm 4.3\end{array}$ & \\
\hline
\end{tabular}

Data are presented as mean \pm standard deviation

D1 Division one competition, D2 Division two competition, D4 Division four competition, Inside power forward and centre positions, $L 2$ second league, Liga ACB Liga Endesa Asociación de Clubs de Baloncesto, Liga EBA Liga Española de Baloncesto Aficionado, NCAA National Collegiate Athletic Association, NR no data provided, Perimeter point guard, shooting guard and small forward positions, PSBC Paulista State Basketball Championships, $U 20$ players competing in an under 20 years of age competition, \# indicates standard error of measurement 
Table 5 Jump height and peak power variables reported during the vertical jump in adult male basketball players

\begin{tabular}{|c|c|c|c|c|c|}
\hline Study & Playing position & Competition level & Category & Jump height $(\mathrm{cm})$ & Peak power $(\mathrm{W})$ \\
\hline Annino et al. [62] & All & Italian National Federal League L2 & Professional & $45.9 \pm 3.4$ & \\
\hline Asadi et al. [97] & All & Provincial D1 Italy & Amateur & $41.3 \pm 3.4$ & \\
\hline Balabinis et al. [98] & All & Undergraduate Teams Greece & Amateur & $52.2 \pm 2.2$ & \\
\hline Balsalobre-Fernandez et al. [99] & All & Spanish Pro Liga ACB & Professional & $45.6 \pm 5.9$ & \\
\hline Balsalobre-Fernandez et al. [100] & All & Spanish Pro Liga ACB & Professional & $43.9 \pm 7.3$ & $4856 \pm 601^{\mathrm{WPD}}$ \\
\hline Buśko et al. [66] & All & Warsaw Sports Club Polonia D2 & Semi-professional & $52.5 \pm 5.1$ & $3591 \pm 788$ \\
\hline Ciacci and Bartolomei [96] & $\begin{array}{l}\text { All } \\
\text { All }\end{array}$ & $\begin{array}{l}\text { National Level Italy } \\
\text { National Level Italy }\end{array}$ & $\begin{array}{l}\text { Professional } \\
\text { Professional }\end{array}$ & $\begin{array}{l}44.6 \pm 6.2 \\
49.3 \pm 5.7\end{array}$ & \\
\hline de Sousa Fortes et al. [59] & All & State Basketball Championship Brazil & Professional & $42.0 \pm 8.0$ & \\
\hline Hoffman et al. [103] & All & NCAA D1 & Collegiate & $64.5 \pm 9.7$ & \\
\hline Hoffman et al. [102] & All & NCAA D1 & Collegiate & $63.4 \pm 6.9$ & \\
\hline Hoffman et al. [104] & $\begin{array}{l}\text { All } 88-89 \\
\text { All } 89-90 \\
\text { All 90-91 } \\
\text { All 91-92 }\end{array}$ & $\begin{array}{l}\text { NCAA D1 } \\
\text { NCAA D1 } \\
\text { NCAA D1 } \\
\text { NCAA D1 }\end{array}$ & $\begin{array}{l}\text { Collegiate } \\
\text { Collegiate } \\
\text { Collegiate } \\
\text { Collegiate }\end{array}$ & $\begin{array}{l}68.1 \pm 8.6 \\
66.0 \pm 6.9 \\
72.6 \pm 5.6 \\
67.3 \pm 6.0\end{array}$ & \\
\hline Hunter et al. [105] & All & U.S College & Collegiate & $61.0 \pm 7.4$ & \\
\hline Kariyawasam et al. [56] & All & National Level Sri Lanka & Professional & $47.9 \pm 6.8$ & \\
\hline Kipp et al. [106] & All & NCAA D1 & Collegiate & $62.4 \pm 5.4$ & \\
\hline Korkmaz and Karahan [107] & $\begin{array}{l}\text { All } \\
\text { All } \\
\text { All }\end{array}$ & $\begin{array}{l}\text { Turkish D1 } \\
\text { Turkish D2 } \\
\text { Turkish D3 (regional) }\end{array}$ & $\begin{array}{l}\text { Professional } \\
\text { Professional } \\
\text { Semi-professional }\end{array}$ & $\begin{array}{l}48.2 \pm 4.0 \\
48.3 \pm 3.0 \\
45.5 \pm 4.0\end{array}$ & $\begin{array}{l}2347 \pm 161 \\
2215 \pm 130 \\
2121 \pm 130\end{array}$ \\
\hline Lehnert et al. [108] & All & Czech First League & Professional & $48.2 \pm 4.6$ & \\
\hline Lockie et al. [3] & $\begin{array}{l}\text { All } \\
\text { Backcourt } \\
\text { Frontcourt }\end{array}$ & $\begin{array}{l}\text { NCAA D1 } \\
\text { NCAA D1 } \\
\text { NCAA D1 }\end{array}$ & $\begin{array}{l}\text { Collegiate } \\
\text { Collegiate } \\
\text { Collegiate }\end{array}$ & $\begin{array}{l}77.9 \pm 9.9 \\
83.4 \pm 8.4 \\
69.9 \pm 5.2\end{array}$ & \\
\hline Mandic et al. [82] & All & National League Serbia & Professional & $43.3 \pm 3.6$ & \\
\hline Montgomery et al. [28] & $\begin{array}{l}\text { All } \\
\text { Guard } \\
\text { Forward } \\
\text { Centre }\end{array}$ & $\begin{array}{l}\text { Australian State Level } \\
\text { Australian State Level } \\
\text { Australian State Level } \\
\text { Australian State Level }\end{array}$ & $\begin{array}{l}\text { Semi-professional } \\
\text { Semi-professional } \\
\text { Semi-professional } \\
\text { Semi-professional }\end{array}$ & $\begin{array}{l}61.9 \pm 14.6 \\
61.3 \pm 19.9 \\
61.2 \pm 7.5 \\
65.3 \pm 9.0\end{array}$ & \\
\hline Nikolaidis et al. [109] & $\begin{array}{l}\text { All } \\
\text { Guard } \\
\text { Forward } \\
\text { Centre }\end{array}$ & $\begin{array}{l}\text { Italian First League } \\
\text { Italian First League } \\
\text { Italian First League } \\
\text { Italian First League }\end{array}$ & $\begin{array}{l}\text { Professional } \\
\text { Professional } \\
\text { Professional } \\
\text { Professional }\end{array}$ & $\begin{array}{l}44.4 \pm 6.8 \\
45.1 \pm 3.3 \\
46.9 \pm 7.8 \\
39.0 \pm 4.3\end{array}$ & \\
\hline Pliaugu et al. [39] & All & Lithuania National Basketball League & Collegiate & $53.0 \pm 9.5^{\mathrm{WPD}}$ & \\
\hline Ponce-González et al. [90] & $\begin{array}{l}\text { All } \\
\text { Guard } \\
\text { Forward } \\
\text { Centre } \\
\text { Perimeter } \\
\text { Inside }\end{array}$ & $\begin{array}{l}\text { Spanish Pro Liga ACB } \\
\text { Spanish Pro Liga ACB } \\
\text { Spanish Pro Liga ACB } \\
\text { Spanish Pro Liga ACB } \\
\text { Spanish Pro Liga ACB } \\
\text { Spanish Pro Liga ACB }\end{array}$ & $\begin{array}{l}\text { Professional } \\
\text { Professional } \\
\text { Professional } \\
\text { Professional } \\
\text { Professional } \\
\text { Professional }\end{array}$ & $\begin{array}{l}44.2 \pm 4.4 \\
43.7 \pm 1.8 \\
43.9 \pm 4.3 \\
45.0 \pm 7.0 \\
44.4 \pm 2.8 \\
43.9 \pm 6.5\end{array}$ & $\begin{array}{l}5753 \pm 932 \\
4992 \pm 546 \\
5567 \pm 606 \\
6701 \pm 709 \\
5300 \pm 663 \\
6388 \pm 931\end{array}$ \\
\hline Puente et al. [14] & $\begin{array}{l}\text { All } \\
\text { Guard } \\
\text { Forward } \\
\text { Centre }\end{array}$ & $\begin{array}{l}\text { National Spanish Basketball Federation } \\
\text { National Spanish Basketball Federation } \\
\text { National Spanish Basketball Federation } \\
\text { National Spanish Basketball Federation }\end{array}$ & $\begin{array}{l}\text { Professional } \\
\text { Professional } \\
\text { Professional } \\
\text { Professional }\end{array}$ & $\begin{array}{l}64 \pm 4 \\
65 \pm 3 \\
64 \pm 6 \\
63 \pm 4\end{array}$ & \\
\hline Rauch et al. [110] & All & NBA & Professional & $68.7 \pm 7.4$ & \\
\hline Rodriguez-Rosell et al. [91] & All & Spanish Liga EBA D4 & Semi-professional & $40.5 \pm 7.0$ & \\
\hline Schiltz et al. [92] & All & Professional D1 European Cup & Professional & $48.7 \pm 5.3$ & \\
\hline Townsend et al. [111] & All & Collegiate D1 United States & Collegiate & $77.4 \pm 6.4$ & \\
\hline
\end{tabular}

Data are presented as mean \pm standard deviation

88-89 data collected from collegiate team during the 1988-90 season, 89-90 data collected from collegiate team during the 1989-90 season, 90-91 data collected from collegiate team during the 1990-91 season, 91-92 data collected from collegiate team during the 1991-2 season, Backcourt players guards, D1 Division one competition, D2 Division two competition, D3 Division three competition, D4 Division four competition, Inside power forward and centre positions, Frontcourt players forwards and centres, L2 second league, Liga ACB Liga Endesa Asociación de Clubs de Baloncesto, Liga EBA Liga Española de Baloncesto Aficionado, NBA National Basketball Association, NCAA National Collegiate Athletic Association, NR no data provided, Perimeter point guard, shooting guard and small forward positions, WPD indicates data extracted by WebPlotDigitizer 
(guards: $3639-4402 \mathrm{~W}$, forwards: $4034-5021 \mathrm{~W}$, and centres: 5054-5149 W) were only reported according to playing position at the professional level. Reliability statistics for each of the jump tests reported in Tables 4, 5 and 6 are provided in Table 13 of the ESM.

\subsection{Linear Sprint Speed}

Linear sprint tests were conducted in 39 (28\%) of the 137
$79,86,91,93,95,112,113]$ and $20 \mathrm{~m}$ (18 studies, $46 \%$ of studies assessing linear sprint speed) $[4,21,28-30,39,51$, $58,59,72,87,89,91,93,95,107,111,114]$. Time was the most common outcome variable and was used in every linear sprint test adopted across studies (Table 5 of the ESM).

Over $5 \mathrm{~m}$, mean sprint times ranged from 0.80 to $1.51 \mathrm{~s}$ (Table 7). Professional players demonstrated mean 5-m sprint times between 0.82 and $1.51 \mathrm{~s}$ (Table 7). Only four studies $[4,21,58,112]$ reported 5-m sprint times at the

Table 6 Jump height and power variables according to playing position and competition level during the squat jump in adult male basketball players

\begin{tabular}{|c|c|c|c|c|c|}
\hline Study & Playing position & Competition level & Category & Jump height $(\mathrm{cm})$ & Peak power $(\mathrm{W})$ \\
\hline Alemdaroglu [61] & All & Turkish D1 & Professional & $32.9 \pm 3.8$ & \\
\hline Aoki et al. [63] & All & Brazilian National League & Professional & $34.8 \pm 2.6$ & \\
\hline Boone and Bourgois [27] & $\begin{array}{l}\text { Point guard } \\
\text { Shooting guard } \\
\text { Small forward } \\
\text { Power forward } \\
\text { Centre }\end{array}$ & $\begin{array}{l}\text { Belgian D1 } \\
\text { Belgian D1 } \\
\text { Belgian D1 } \\
\text { Belgian D1 } \\
\text { Belgian D1 }\end{array}$ & $\begin{array}{l}\text { Professional } \\
\text { Professional } \\
\text { Professional } \\
\text { Professional } \\
\text { Professional }\end{array}$ & $\begin{array}{l}41.0 \pm 3.8 \\
39.5 \pm 3.6 \\
40.2 \pm 3.7 \\
39.1 \pm 4.2 \\
35.7 \pm 3.2\end{array}$ & $\begin{array}{l}4203 \pm 371 \\
4402 \pm 358 \\
4761 \pm 381 \\
5021 \pm 423 \\
5149 \pm 399\end{array}$ \\
\hline Chaouachi et al. [67] & All & Tunisian National Team & Professional & $49.5 \pm 4.8$ & \\
\hline Ciacci and Bartolomei [96] & $\begin{array}{l}\text { All } \\
\text { All }\end{array}$ & $\begin{array}{l}\text { National Level Italy } \\
\text { National Level Italy }\end{array}$ & $\begin{array}{l}\text { Professional } \\
\text { Professional }\end{array}$ & $\begin{array}{l}36.2 \pm 5.0 \\
42.5 \pm 5.0\end{array}$ & \\
\hline Gomes et al. [72] & All & PSBC & Professional & $33.4 \pm 5.2$ & \\
\hline Jallai et al. [77] & All & Estonian 1st League & Professional & $40.4 \pm 2.0$ & $24.23 \pm 5.77^{\wedge}$ \\
\hline Khlifa et al. [78] & All & Tunisian D1 & Professional & $38.6 \pm 1.1^{\#}$ & \\
\hline Köklü et al. [79] & $\begin{array}{l}\text { All } \\
\text { All } \\
\text { All } \\
\text { Guard } \\
\text { Forward } \\
\text { Centre }\end{array}$ & $\begin{array}{l}\text { Turkish D1 and D2 } \\
\text { Turkish D1 } \\
\text { Turkish D2 } \\
\text { Turkish D1 and D2 } \\
\text { Turkish D1 and D2 } \\
\text { Turkish D1 and D2 }\end{array}$ & $\begin{array}{l}\text { Professional } \\
\text { Professional } \\
\text { Professional } \\
\text { Professional } \\
\text { Professional } \\
\text { Professional }\end{array}$ & $\begin{array}{l}36.2 \pm 5.5 \\
37.8 \pm 5.7 \\
34.7 \pm 5.7 \\
36.4 \pm 5.7 \\
37.7 \pm 5.2 \\
34.7 \pm 5.4\end{array}$ & \\
\hline Maffiuletti et al. [81] & All & French Basketball Federation D2 & Professional & $44.1 \pm 1.8$ & \\
\hline Maggioni et al. [58] & All & Volunteer Players & Semi-professional & $26.5 \pm 3.8$ & \\
\hline Pojskic et al. [89] & All & Bosnia and Herzegovina D1 & Professional & $31.1 \pm 31.1$ & \\
\hline Ponce-Gonzalez et al. [90] & $\begin{array}{l}\text { All } \\
\text { Guard } \\
\text { Forward } \\
\text { Centre } \\
\text { Perimeter } \\
\text { Inside }\end{array}$ & $\begin{array}{l}\text { Spanish Pro Liga ACB } \\
\text { Spanish Pro Liga ACB } \\
\text { Spanish Pro Liga ACB } \\
\text { Spanish Pro Liga ACB } \\
\text { Spanish Pro Liga ACB } \\
\text { Spanish Pro Liga ACB }\end{array}$ & $\begin{array}{l}\text { Professional } \\
\text { Professional } \\
\text { Professional } \\
\text { Professional } \\
\text { Professional } \\
\text { Professional }\end{array}$ & $\begin{array}{l}30.6 \pm 5.5 \\
30.1 \pm 5.7 \\
28.5 \pm 3.2 \\
33.2 \pm 7.3 \\
29.6 \pm 4.6 \\
31.9 \pm 6.8\end{array}$ & $\begin{array}{l}4242 \pm 746 \\
3639 \pm 413 \\
4034 \pm 390 \\
5054 \pm 544 \\
3872 \pm 449 \\
4761 \pm 807\end{array}$ \\
\hline Shalfawi et al. [93] & All & Professional level Norway & Professional & $43.1 \pm 7.2$ & $4609 \pm 419$ \\
\hline Xie et al. [95] & $\begin{array}{l}\text { All } \\
\text { All }\end{array}$ & $\begin{array}{l}\text { NCAA D1 university team } \\
\text { NCAA D1 club team }\end{array}$ & $\begin{array}{l}\text { Collegiate } \\
\text { Collegiate }\end{array}$ & $\begin{array}{l}57.8 \pm 7.9 \\
56.4 \pm 3.7\end{array}$ & \\
\hline
\end{tabular}

Data are presented as mean \pm standard deviation

D1 Division one competition, D2 Division two competition, Inside power forward and centre positions, Liga ACB Liga Endesa Asociación de Clubs de Baloncesto, NCAA National Collegiate Athletic Association, Perimeter point guard, shooting guard and small forward positions, PSBC Paulista State Basketball Championships, ^ indicates reported in $\mathrm{W} \cdot \mathrm{kg}^{-1}$, \# indicates standard error of measurement

studies included in this review (Table 5 of the ESM). The most frequently included linear sprint distances were $5 \mathrm{~m}(9$ studies, $23 \%$ of studies assessing linear sprint speed) $[4,21$, $27,51,58,64,65,67,112], 10 \mathrm{~m}$ (16 studies, $41 \%$ of studies assessing linear sprint speed) $[21,27,60,61,64,65,67,71$, semi-professional level (1.04-1.14 s). One study reported 5-m sprint time in representative [65] and collegiate [51] players (Table 7). No studies reported 5-m sprint times in amateur players. Mean sprint times over $10 \mathrm{~m}$ ranged from 1.47 to $2.34 \mathrm{~s}$ (Table 7). Professional players recorded mean 
10-m sprint times between 1.47 and $2.34 \mathrm{~s}$. Homogenous mean 10-m sprint times were reported in semi-professional players (1.77-1.90 s). Only one study [113] reported 10-m sprint time in amateur players (Table 7). Mean sprint times over $20 \mathrm{~m}$ ranged between 2.43 and $3.36 \mathrm{~s}$ (Table 7). Mean sprint times over $20 \mathrm{~m}$ were similar between professional (2.43-3.24 s) and semi-professional (2.80-3.24 s) players. One study [114] reported 20-m sprint times in representative players (Table 7). Collegiate players recorded mean 20 -m sprint times ranging from 2.80 to $3.36 \mathrm{~s}$. No studies reported 20-m sprint time in amateur players. Linear sprint performance according to playing position was reported at the professional [27] and semi-professional [4] levels across $5 \mathrm{~m}$, professional [27, 79] and semi-professional [4] levels across $10 \mathrm{~m}$, and professional $[87,89]$ and semi-professional [4, 28] levels across $20 \mathrm{~m}$ (Table 7). Reliability statistics for each of the linear sprint tests described in Table 7 are provided in Table 13 of the ESM.

\subsection{Change-of-Direction Speed}

Change-of-direction speed was assessed in 38 (28\%) of the 137 studies in this review, with 17 different tests used (Table 6 of the ESM). All tests used time as the primary outcome variable except the Multi-Stage Change of Direction Exercise Test, which used metabolic power, running speed, peak torque and fatigue index as outcome variables (Table 6 of the ESM). The Agility $T$-Test was the most frequently implemented change-of-direction speed test being used in 20 studies (53\% of studies measuring change-of-direction speed) $[49,58,61,65,67,70-72,79,87,89,97,102-104$, 112, 114-117]. Mean Agility $T$-Test time ranged between 8.84 and $10.90 \mathrm{~s}$ across studies. Professional players demonstrated mean Agility $T$-Test times between 8.84 and $10.04 \mathrm{~s}$, which were similar to collegiate players (8.92-9.78 s), and quicker than mean times reported in semi-professional players $(9.52-10.90 \mathrm{~s})$. Agility $T$-Test time was only reported in representative players $(9.21-10.05 \mathrm{~s})$ in two studies [65, 114] while only one study [97] observed amateur players (Table 8 ). Two studies $[49,79]$ reported Agility $T$-Test times relative to playing position (guard: $8.96-9.24 \mathrm{~s}$, forward: $8.84-9.48 \mathrm{~s}$, and centre: $9.73-10.04 \mathrm{~s}$ ), only at the professional level. The Lane Agility Test was only used in three studies ( $8 \%$ of studies measuring change-of-direction speed) $[3,51,111]$ and only assessing collegiate players (10.16-11.80 s), with one study [3] reporting results according to playing position (Table 8 ). Table 8 contains Agility $T$-Test, Lane Agility Test and Y-Shaped Change-of-Direction Speed Test outcomes reported in adult male basketball players. Reliability statistics for each of the change-of-direction tests described in Table 8 are provided in Table 13 of the ESM.

\subsection{Agility}

Agility performance was reported in seven (5\%) of the 137 studies included in this review (Table 7 of the ESM). Only three tests were used to assess agility including the Reactive Agility Test [4, 21, 60], Reactive Change-of-Direction Test [113, 118], and Reactive Y-Shaped Change-of-Direction Test $[49,85]$. Time was the primary outcome variable reported across studies in all agility tests, with response time and decision-making time also reported in three studies [4, $21,60]$. Agility tests were performed slower compared to the pre-determined change-of-direction speed tests following the same design [4, 21, 49, 60, 113, 118]. The Reactive Agility Test was performed exclusively at the semi-professional level $[4,21,60]$ and performance ranged between 2.00 and $2.18 \mathrm{~s}$. Reactive COD Test performance ranged between 2.52 and $2.77 \mathrm{~s}$ at the semi-professional $[113,118]$ level. Only one study [113] reported Reactive COD performance at the amateur level (Table 9). The Reactive Y-COD test was only reported in two studies [48, 49] (Table 9). No studies measured the agility of players at collegiate or representative levels. Only one study [49] reported agility performance by playing position (Table 9). Reliability statistics for each of the agility tests described in Table 9 are provided in Table 13 of the ESM.

\subsection{Strength}

Strength testing was undertaken in 42 (31\%) of the 137 studies in this review (Table 8 of the ESM). Repetition maximum outcome variables were most frequently gathered across studies, with 1RM and 3RM being the most used protocols (Table 8 of the ESM). Bench press performance, represented by 1 RM were observed in 17 studies ( $40 \%$ of studies assessing strength) $[51,56,65,67,70-72,98,99,102-105,114$, 119-121], with mean loads lifted between 70 and $112 \mathrm{~kg}$ (Table 10). Professional players bench pressed 1RM loads between 70 and $112 \mathrm{~kg}$ (Table 10). Only two studies reported bench press 1RM each in semi-professional $(76-86 \mathrm{~kg})[70$, $71]$ and representative $(77-101 \mathrm{~kg})[65,114]$ players. Collegiate players bench pressed 1RM loads between 76 and $102 \mathrm{~kg}$ (Table 10). Only one study [98] reported bench press $1 \mathrm{RM}$ in amateur players and only one study [121] reported bench press 1RM by playing position, at the professional level (Table 10).

The squat exercise (i.e., front and back squat) was used in 16 studies (38\% of studies assessing strength) [51, 59, $65,67,70,71,91,98,102-104,111,119,122-124]$ to assess strength with 1RM and 3RM protocols most frequently used (Table 8 of the ESM). Mean back squat 1RM loads ranged between 116 and $202 \mathrm{~kg}$ across studies (Table 10). Professional players squatted greater mean 1RM loads $(143-202 \mathrm{~kg})$ than collegiate players $(116-156 \mathrm{~kg})$ 
Table 7 Sprint times recorded during 5-m, 10-m and 20-m linear sprints in adult male basketball players

\begin{tabular}{|c|c|c|c|c|c|c|}
\hline Study & Playing position & Competition level & Category & $\begin{array}{l}\text { 5-m sprint } \\
\text { time (s) }\end{array}$ & $\begin{array}{l}\text { 10-m sprint } \\
\text { time }(\mathrm{s})\end{array}$ & $\begin{array}{l}20-\mathrm{m} \text { sprint } \\
\text { time }(\mathrm{s})\end{array}$ \\
\hline Alemdaroglu [61] & All & Turkish D1 & Professional & & $1.86 \pm 0.30$ & \\
\hline $\begin{array}{l}\text { Barrera-Domínguez et al. } \\
\text { [64] }\end{array}$ & All & Spanish National Division & Professional & $0.88 \pm 0.04$ & $1.47 \pm 0.08$ & \\
\hline \multirow[t]{2}{*}{ Ben Abdelkrim et al. [65] } & All (U20) & Tunisian National Team & Representative & $1.00 \pm 0.10$ & & \\
\hline & All & Tunisian National Team & Professional & $1.04 \pm 0.16$ & $\begin{array}{l}1.84 \pm 0.10 \\
1.88 \pm 0.15\end{array}$ & \\
\hline \multirow[t]{5}{*}{ Boone and Bourgois [27] } & Point guard & Belgian D1 & Professional & $1.40 \pm 0.03$ & $2.16 \pm 0.09$ & \\
\hline & Shooting guard & Belgian D1 & Professional & $1.40 \pm 0.09$ & $2.19 \pm 0.08$ & \\
\hline & Small forward & Belgian D1 & Professional & $1.45 \pm 0.09$ & $2.23 \pm 0.09$ & \\
\hline & Power forward & Belgian D1 & Professional & $1.47 \pm 0.08$ & $2.25 \pm 0.08$ & \\
\hline & Centre & Belgian D1 & Professional & $1.51 \pm 0.07$ & $2.34 \pm 0.11$ & \\
\hline Chaouachi et al. [67] & All & Tunisian National Team & Professional & $0.82 \pm 0.05$ & $1.70 \pm 0.06$ & \\
\hline Dawes and Spiteri [51] & All & NCAA D2 & Collegiate & $0.80 \pm 0.04$ & & $2.80 \pm 0.08$ \\
\hline de Sousa Fortes et al. [59] & All & State Basketball Championship Brazil & Professional & & & $2.43 \pm 0.21$ \\
\hline Delextrat and Cohen [114] & $\begin{array}{l}\text { All } \\
\text { All } \\
\text { All }\end{array}$ & $\begin{array}{l}\text { BUSA D1 and D2 } \\
\text { BUSA D1 } \\
\text { BUSA D2 }\end{array}$ & $\begin{array}{l}\text { Collegiate-Representative } \\
\text { Representative } \\
\text { Collegiate }\end{array}$ & & & $\begin{array}{l}3.33 \pm 0.26 \\
3.29 \pm 0.12 \\
3.36 \pm 0.36\end{array}$ \\
\hline Freitas et al. [71] & All & Spanish Liga EBA D4 & Semi-professional & & $1.91 \pm 0.09$ & \\
\hline Gomes et al. [72] & All & PSBC & Professional & & & $3.24 \pm 0.22$ \\
\hline \multirow[t]{6}{*}{ Köklü et al. [79] } & All & Turkish D1 and D2 & Professional & & $1.75 \pm 0.08$ & \\
\hline & All & Turkish D1 & Professional & & $1.78 \pm 0.80$ & \\
\hline & All & Turkish D2 & Professional & & $1.72 \pm 0.80$ & \\
\hline & Guards & Turkish D1 and D2 & Professional & & $1.72 \pm 0.07$ & \\
\hline & Forward & Turkish D1 and D2 & Professional & & $1.72 \pm 0.07$ & \\
\hline & Centre & Turkish D1 and D2 & Professional & & $1.80 \pm 0.08$ & \\
\hline \multirow[t]{3}{*}{ Korkmaz and Karahan [107] } & All & Turkish D1 & Professional & & & $2.70 \pm 0.14$ \\
\hline & All & Turkish D2 & Professional & & & $2.80 \pm 0.10$ \\
\hline & All & Turkish D3 & Semi-professional & & & $2.80 \pm 0.13$ \\
\hline \multirow[t]{2}{*}{ Lockie et al. [113] } & All & Australian State level & Semi-professional & & $1.81 \pm 0.09$ & \\
\hline & All & Recreational Australia & Amateur & & $1.88 \pm 0.07$ & \\
\hline Maggioni et al. [58] & All & Volunteer Players & Semi-professional & $1.04 \pm 0.05$ & $1.77 \pm 0.04$ & $3.10 \pm 0.12$ \\
\hline \multirow[t]{4}{*}{ Montgomery et al. [28] } & All & Australian State Level & Semi-professional & & & $3.09 \pm 0.10$ \\
\hline & Guard & Australian State Level & Semi-professional & & & $3.04 \pm 0.07$ \\
\hline & Forward & Australian State Level & Semi-professional & & & $3.13 \pm 0.13$ \\
\hline & Centre & Australian State Level & Semi-professional & & & $3.10 \pm 0.09$ \\
\hline Pliauga et al. [39] & All & Lithuanian National Basketball League & Collegiate & & & $2.98 \pm 0.32$ \\
\hline Pliauga et al. [86] & All & Lithuanian National Basketball League & Collegiate & & $1.80 \pm 0.03$ & \\
\hline Pojskić et al. [87] & Perimeter & Bosnia and Herzegovina D1 & Professional & & & $3.14 \pm 0.09$ \\
\hline Pojskić et al. [89] & Perimeter & Bosnian Premier League & Professional & & & $3.14 \pm 0.09$ \\
\hline Poole et al. [112] & All & Australian State level QBL & Semi-professional & $1.14 \pm 0.07$ & $1.90 \pm 0.10$ & \\
\hline Ramirez-Campillo et al. [29] & All & SCCSBL & Collegiate & & & $3.00 \pm 0.11$ \\
\hline Rodriguez-Rosell et al. [91] & All & Spanish Liga EBA D4 & Semi-professional & & $1.84 \pm 0.10$ & $3.20 \pm 0.16$ \\
\hline Scanlan et al. [21] & All & Australian State Level & Semi-professional & $1.07 \pm 0.07$ & $1.83 \pm 0.10$ & $3.16 \pm 0.19$ \\
\hline \multirow[t]{2}{*}{ Scanlan et al. [4] } & Backcourt & Australian State Level & Semi-professional & $1.05 \pm 0.03$ & $1.78 \pm 0.05$ & $3.08 \pm 0.12$ \\
\hline & Frontcourt & Australian State Level & Semi-professional & $1.10 \pm 0.09$ & $1.87 \pm 0.13$ & \\
\hline Scanlan et al. [30] & All & Australian State Level & Semi-professional & & & $\begin{array}{l}3.24 \pm 0.22 \\
2.97 \pm 0.06\end{array}$ \\
\hline Shalfawi et al. [93] & All & Professional Level Norway & Professional & & $1.88 \pm 0.21$ & $3.20 \pm 0.33$ \\
\hline Townsend et al. [111] & All & Collegiate D1 United States & Collegiate & & & $3.31 \pm 0.15^{1080}$ \\
\hline Xie et al. [95] & All & NCAA D1 university and club teams & Collegiate & & $5.67 \pm 0.17^{\mathrm{m} / \mathrm{s}}$ & $7.94 \pm 0.45^{\mathrm{m} / \mathrm{s}}$ \\
\hline
\end{tabular}

Data presented as mean \pm standard deviation

BUSA British Universities Sports Association, D1 Division one competition, D2 Division two competition, D3 Division three competition, D4 Division four competition, Liga EBA Liga Española de Baloncesto Aficionado, NCAA National Collegiate Athletic Association, Perimeter point guard, shooting guard and small forward positions, PSBC Paulista State Basketball Championships, SCCSBL South Chilean College System Basketball League, $U 20$ players competing in an under 20 years of age competition, ${ }^{\mathrm{m} / \mathrm{s}}$ indicates velocity in $\mathrm{m}^{-1} \mathrm{~s}^{-1},{ }^{1080}$ indicates performed using 1080 sprint with $1-\mathrm{kg}$ resistance 
(Table 10). Only two studies $[70,71]$ reported back squat 1RM loads in semi-professional players (149-157 kg), while one study [65] assessed back squat 1RM load in representative players. No studies reported back squat performance in amateur players or relative to playing position. All additional strength tests and outcome variables are reported in Table 8 of the ESM, while the bench press and squat outcome variables reported in individual studies are shown in Table 10. Reliability statistics for each of the strength tests described in Table 10 are provided in Table 13 of the ESM.

\subsection{Anaerobic Capacity}

Anaerobic capacity was assessed in 35 studies (26\%) of the 137 studies in this review, using 20 different tests (Table 9 of the ESM). The most frequently implemented tests were the WAnT (nine studies, $26 \%$ of studies assessing anaerobic capacity) [61, 98, 109, 114, 117, 125-128], the RAST (six studies, $18 \%$ of studies assessing anaerobic capacity) $[87-89,99,100,129]$, and the full court shuttle run (five studies, $14 \%$ of studies assessing anaerobic capacity) [28, 51, 58, 116, 125]. Peak power, mean power, fatigue index, and time were the most reported outcome variables (Table 11). Performance during the WAnT was reported in seven studies $[61,109,117,125-128]$ at the professional level (mean power: 683-823 W, peak power: 951-1085 W, and fatigue index: 43-60\%). However, only one study reported WAnT performance each in representative [114] and collegiate [114] players, while two studies [98, 125] were observed at the amateur level (Table 11). No studies reported WAnT performance in semi-professional players. Two studies $[109,126]$ reported WAnT performance according to playing position (guards: peak power: $11-13 \mathrm{~W} / \mathrm{kg}$, fatigue index: $48-64 \%$, forwards: peak power: $11-13 \mathrm{~W} / \mathrm{kg}$, fatigue index: $43-58 \%$, centres: $10-11 \mathrm{~W} / \mathrm{kg}$, fatigue index: 44-56\%). The RAST was only reported in professional players [87-89, 99, 100, 129], with mean peak power ranging between 761 and $957 \mathrm{~W}$ and mean power between 608 and $772 \mathrm{~W}$. Two studies $[88,129]$ reported RAST performance according to playing position. Four studies used the full court shuttle run, with professional [125], semi-professional $[28,58]$, collegiate [51] and amateur [125] players assessed (Table 11). The full court shuttle run was only reported relative to playing position in one study [28] consisting of semiprofessional players (Table 11). Reliability statistics for each of the anaerobic capacity tests described in Table 11 are provided in Table 13 of the ESM.

\subsection{Aerobic Capacity}

Aerobic capacity was assessed in 57 (42\%) of the 137 studies included in this review, with 14 different tests used (Table 10 of the ESM). Incremental treadmill tests (17 studies, 30\% of studies assessing aerobic capacity), which involved the Bruce $[105,120,130]$ and various incremental running protocols [27, 57-59, 90, 94, 121, 125, 126, 131-135], as well as the Yo-Yo IRL1 (14 studies, 25\% of studies assessing aerobic capacity) $[4,16,21,28,55,58,60,63,65,67,72$, 136-138] and MSFT (eight studies, 14\% of studies assessing aerobic capacity) $[51,61,79,84,87-89,107]$ were the most frequently used tests. The most common outcome variable reported from aerobic testing was maximum oxygen uptake $\left(\mathrm{VO}_{2 \max }\right)$ [Table 10 of the ESM]. However, if during a maximal test the criteria for $\mathrm{VO}_{2 \max }$ was not achieved, $\mathrm{VO}_{2 \text { peak }}$ was reported as the outcome variable [27, 131, 134].

Mean aerobic capacity during incremental treadmill tests ranged from 42 to $61 \mathrm{~mL} / \mathrm{kg} / \mathrm{min}$ across studies (Table 12). The mean aerobic capacity of professional players ranged between 42 and $61 \mathrm{~mL} / \mathrm{kg} / \mathrm{min}$. Only one study [135] assessed aerobic capacity in semi-professional players using an incremental treadmill test, while no studies assessed representative players. Mean aerobic capacity in collegiate players ranged between 50 and $58 \mathrm{~mL} / \mathrm{kg} / \mathrm{min}$. Aerobic capacity in amateur players were only assessed in two studies [125, 135] using an incremental treadmill test. When observing aerobic capacity according to playing position using incremental treadmill tests, only professional player data were apparent $[27,90,121,126,131]$ with mean aerobic capacity in guards ranging between 50 and $58 \mathrm{~mL} / \mathrm{kg} / \mathrm{min}$, in forwards between 46 and $58 \mathrm{~mL} / \mathrm{kg} / \mathrm{min}$ and in centres between 42 and $58 \mathrm{~mL} / \mathrm{kg} / \mathrm{min}$.

Estimated $\mathrm{VO}_{2 \max }$ from the MSFT ranged from 42 to $64 \mathrm{ml} / \mathrm{kg} / \mathrm{min}$, while the number of shuttles completed were between 66 and 106 across studies (Table 12). The $\mathrm{VO}_{2 \max }$ of professional players ranged from 42 to $64 \mathrm{~mL} / \mathrm{kg} / \mathrm{min}$ using the MSFT. Only one study reported $\mathrm{VO}_{2 \max }$ during the MSFT each in semi-professional [107] and collegiate [61] players (Table 12). No studies reported MSFT in representative or amateur players. Mean estimated $\mathrm{VO}_{2 \max }$ relative to playing position using the MSFT was only reported in professional players $[79,84,88]$ with guards $(45-64 \mathrm{~mL} / \mathrm{kg} /$ $\mathrm{min}$ ), forwards ( $43-62 \mathrm{~mL} / \mathrm{kg} / \mathrm{min}$ ) and centres $(42-58 \mathrm{~mL} /$ $\mathrm{kg} / \mathrm{min}$ ) showing similar data.

Mean estimated $\mathrm{VO}_{2 \max }$ derived during the Yo-Yo IRL1 ranged between 47 and $60 \mathrm{~mL} / \mathrm{kg} / \mathrm{min}$, while mean distances reached were between 636 and $2447 \mathrm{~m}$ across studies (Table 12). The $\mathrm{VO}_{2 \max }$ of professional players using the Yo-Yo IRL1 $(47-60 \mathrm{~mL} / \mathrm{kg} / \mathrm{min})$ was only reported in three studies [65, 67, 72], while the mean distance covered ranged between 1120 and $2389 \mathrm{~m}$. The $\mathrm{VO}_{2 \max }$ of semiprofessional players using the Yo-Yo IRL1 $(48-52 \mathrm{~mL} / \mathrm{kg} /$ min) was reported in three studies $[4,21,60]$, while mean distances covered ranged from 996 to $2265 \mathrm{~m}$. $\mathrm{VO}_{2 \max }$ was only observed in one study using the Yo-Yo IRL1 in representative players [65] (Table 12). No studies used the Yo-Yo IRL1 to estimate $\mathrm{VO}_{2 \max }$ in collegiate players, while two 
Table 8 COD speed test performance according to playing position and competition level in adult male basketball players

\begin{tabular}{|c|c|c|c|c|c|}
\hline Study & Playing position & Competition level & Category & Test & Time (s) \\
\hline Alembaroglu [61] & All & Turkish D1 & Professional & Agility $T$-Test & $9.25 \pm 0.46$ \\
\hline Asadi et al. [97] & All & Provincial D1 Italy & Amateur & Agility $T$-Test & $12.00 \pm 0.56$ \\
\hline Barrera-Domínguez et al. [64] & All & Spanish National Division & Professional & Modified $T$-Test & $6.49 \pm 0.34$ \\
\hline \multirow[t]{2}{*}{ Ben Abdelkrim et al. [65] } & All (U20) & Tunisian National Team & Representative & Agility $T$-Test & $10.05 \pm 0.44$ \\
\hline & All & Tunisian National Team & Professional & Agility $T$-Test & $9.99 \pm 0.40$ \\
\hline Chaouachi et al. [67] & All & Tunisian National Team & Professional & Agility $T$-Test & $9.70 \pm 0.20$ \\
\hline \multirow[t]{3}{*}{ Delextrat and Cohen [114] } & All & BUSA D1 and D2 & Collegiate - Representative & Agility $T$-Test & $9.49 \pm 0.56$ \\
\hline & All & BUSA D1 & Representative & Agility $T$-Test & $9.21 \pm 0.24$ \\
\hline & All & BUSA D2 & Collegiate & Agility $T$-Test & $9.78 \pm 0.59$ \\
\hline Freitas et al. [70] & All & Spanish Liga EBA D4 & Semi-professional & Agility $T$-Test & $9.52 \pm 0.63$ \\
\hline Freitas et al. [71] & All & Spanish Liga EBA D4 & Semi-professional & Agility $T$-Test & $9.71 \pm 0.67$ \\
\hline Gomes et al. [72] & All & PSBC & Professional & Agility $T$-Test & $9.28 \pm 0.46$ \\
\hline Hoffman et al. [102] & All & NCAA D1 & Collegiate & Agility $T$-Test & $8.92 \pm 0.30$ \\
\hline Hoffman et al. [103] & All & NCAA D1 & Collegiate & Agility $T$-Test & $9.18 \pm 0.54$ \\
\hline \multirow[t]{4}{*}{ Hoffman et al. [104] } & All 88-89 & NCAA D1 & Collegiate & Agility $T$-Test & $9.11 \pm 0.46$ \\
\hline & All 89-90 & NCAA D1 & Collegiate & Agility $T$-Test & $8.94 \pm 0.34$ \\
\hline & All 90-91 & NCAA D1 & Collegiate & Agility $T$-Test & $9.00 \pm 0.45$ \\
\hline & All 91-92 & NCAA D1 & Collegiate & Agility $T$-Test & $9.15 \pm 0.41$ \\
\hline \multirow[t]{6}{*}{ Köklü et al. [79] } & All & Turkish D1 & Professional & Agility $T$-Test & $9.49 \pm 0.61$ \\
\hline & All & Turkish D2 & Professional & Agility $T$-Test & $9.76 \pm 0.57$ \\
\hline & All & Turkish D1 and D2 & Professional & Agility $T$-Test & $9.61 \pm 0.57$ \\
\hline & Guard & Turkish D1 and D2 & Professional & Agility $T$-Test & $9.24 \pm 0.56$ \\
\hline & Forward & Turkish D1 and D2 & Professional & Agility $T$-Test & $9.48 \pm 0.46$ \\
\hline & Centre & Turkish D1 and D2 & Professional & Agility $T$-Test & $10.04 \pm 0.35$ \\
\hline Lehnert et al. [108] & All & Czech First League & Professional & Agility $T$-Test & $9.35 \pm 0.49$ \\
\hline Maggioni et al. [58] & All & Volunteer Players & Semi-professional & Agility $T$-Test & $9.8 \pm 0.2$ \\
\hline Pojskić et al. [89] & Perimeter & Bosnia and Herzegovina D1 & Professional & Agility $T$-Test & $10.48 \pm 0.41$ \\
\hline Poole et al. [112] & All & Australian State Level & Semi-professional & Agility $T$-Test & $10.90 \pm 0.51$ \\
\hline \multirow[t]{5}{*}{ Sekulic et al. [49] } & Guard & Bosnia and Herzegovina D1 and D2 & Professional & Agility $T$-Test & $8.96 \pm 0.37$ \\
\hline & Forward & Bosnia and Herzegovina D1 and D2 & Professional & Agility $T$-Test & $8.84 \pm 0.34$ \\
\hline & Centre & Bosnia and Herzegovina D1 and D2 & Professional & Agility $T$-Test & $9.73 \pm 0.50$ \\
\hline & All & Bosnia and Herzegovina D1 & Professional & Agility $T$-Test & $9.02 \pm 0.49$ \\
\hline & All & Bosnia and Herzegovina D2 & Professional & Agility $T$-Test & $9.14 \pm 0.43$ \\
\hline Dawes and Spiteri [51] & All & NCAA D2 & Collegiate & Lane Agility Test & $11.24 \pm 0.54$ \\
\hline \multirow[t]{3}{*}{ Lockie et al. [3] } & All & NCAA D1 & Collegiate & Lane Agility Test & $10.42 \pm 0.61$ \\
\hline & Frontcourt & NCAA D1 & Collegiate & Lane Agility Test & $10.95 \pm 0.78$ \\
\hline & Backcourt & NCAA D1 & Collegiate & Lane Agility Test & $10.16 \pm 0.33$ \\
\hline Townsend et al. [111] & All & Collegiate D1 United States & Collegiate & Lane Agility Test & $11.80 \pm 0.90$ \\
\hline \multirow[t]{4}{*}{ Lockie et al. [113] } & All & Semi-Professional & Semi-professional & COD Left foot start & $1.88 \pm 0.09$ \\
\hline & All & Semi-Professional & Semi-professional & COD Right foot start & $1.88 \pm 0.14$ \\
\hline & All & Recreational & Amateur & COD Left foot start & $1.94 \pm 0.12$ \\
\hline & All & Recreational & Amateur & COD Right foot start & $1.96 \pm 0.14$ \\
\hline Pehar et al. [85] & All & Bosnia and Herzegovina D1 & Professional & Y-COD & $1.68 \pm 0.15$ \\
\hline \multirow[t]{3}{*}{ Scanlan et al. [60] } & All & Australian State Level & Semi-professional & Y-CODST & $1.64 \pm 0.10$ \\
\hline & All STARTERS & Australian State Level & Semi-professional & Y-CODST & $1.65 \pm 0.11$ \\
\hline & All NON-STARTERS & Australian State Level & Semi-professional & Y-CODST & $1.63 \pm 0.10$ \\
\hline Scanlan et al. [21] & All & Australian State Level & Semi-professional & Y-CODST & $1.64 \pm 0.10$ \\
\hline \multirow[t]{2}{*}{ Scanlan et al. [4] } & Backcourt & Australian State Level & Semi-professional & Y-CODST & $1.67 \pm 0.10$ \\
\hline & Frontcourt & Australian State Level & Semi-professional & Y-CODST & $1.61 \pm 0.11$ \\
\hline
\end{tabular}

Data presented as mean \pm standard deviation

88-89 data collected from collegiate team during the 1988-90 season, 89-90 data collected from collegiate team during the 1989-90 season, 90-91 ata collected from collegiate team during the 1990-91 season, 91-92 data collected from collegiate team during the 1991-2 season, Backcourt players guards, BUSA British Universities Sports Association, COD change-of-direction, CODST Change-of-Direction Speed Test, D1 Division one competition, D2 Division two competition, D4 Division four competition, Frontcourt players forwards and centres, Liga EBA Liga 
Table 8 (continued)

Española de Baloncesto Aficionado, NCAA National Collegiate Athletic Association, perimeter point guard, shooting guard and small forward positions, $P S B C$ Paulista State Basketball Championships, $U 20$ under 20 years of age competition

Modified $T$-Test requires players to sprint $5 \mathrm{~m}$ forward, shuffle $2.5 \mathrm{~m}$ laterally to the left, then shuffle $5 \mathrm{~m}$ to the right, shuffle $2.5 \mathrm{~m}$ to the left and then backpedal to the starting position

studies $[16,137]$ reported distance using the Yo-Yo IRL1 in amateur players (636-1671 m). Yo-Yo IRL1 performance relative to playing position was only reported in semi-professional players using estimated $\mathrm{VO}_{2 \max }$ [4] and distance [28], and in one study [16], which reported the mean across amateur, semi-professional, and professional players (Table 12). Reliability statistics for each of the aerobic capacity tests described in Table 12 are provided in Table 13 of the ESM.

\section{Discussion}

The aims of this systematic review were to (1) identify tests and outcome variables used to assess physical characteristics in adult male basketball players across all competition levels, (2) report a summary of anthropometric, muscular power, linear speed, change-of-direction speed, agility, strength, anaerobic capacity, and aerobic capacity in adult male basketball players based on playing position and competition level, and (3) introduce a framework outlining recommended testing approaches to quantify physical characteristics in adult male basketball players. As expected, the number of tests and outcome variables reported reveal extensive variability in how the physical characteristics of adult male basketball players are tested. An indirect finding of this review was the wide range of approaches and variability in procedures and calculations of basic outputs (e.g., jump height measured through flight time vs take-off velocity). Additionally, the validity and reliability statistics of commonly used tests were often not reported. Thus, it is difficult to draw firm conclusions about the physical characteristics of basketball players. The wide-ranging physical performances observed were likely influenced by the choice of test and methodology employed by researchers. These issues have made it difficult to establish consensus based on the basketball literature. To improve the overall understanding of the physical characteristics required to excel at different competition levels in adult male basketball, researchers and practitioners should consider: (1) the validity, reliability and standardisation of tests being employed; (2) the appropriateness and specificity of the tests being implemented; and (3) the ability of testing information to discern players of different playing positions and competition levels. It is important to ensure outcome variables gathered are valid and reliable in order to detect meaningful changes over time. As each test may have an inherent level of variability or 'noise', discerning changes that are practically relevant is critical. Particularly when using data to quantify player progression, or to use performance data gathered during a test to guide rehabilitation, or when monitoring performance and fatigue. Furthermore, it is advised that researchers attempt to align with practitioners to continue to develop standardised testing batteries (e.g., NBA draft combine) that optimally support the profiling of adult male basketball players.

\subsection{Tests and Outcome Variables}

Anthropometric values of height and body mass were reported in all 114 studies eligible to address the second aim of this review. Body composition was primarily measured using low-cost, easy to implement tests, such as sum of skinfold measurements. Furthermore, muscular power was most commonly measured indirectly with a combination of three bilateral jumps (i.e., CMJ, CJ and SJ) that provide insight into varying speed-strength jump qualities $[78,139]$. Linear sprint performances were primarily reported over distances of 5, 10, and $20 \mathrm{~m}$. Of those three distances, $5 \mathrm{~m}$, which is reflective of an athlete's ability to accelerate and is similar to movements frequently encountered during match-play, was the least reported. The Agility $T$-Test was the most commonly used test to assess change-of-direction speed, potentially because of the ease of implementation and inclusion of basketballspecific lateral movements. However, in recent years, collegiate-level players from the NCAA were observed using the Lane Agility Test to assess change-of-direction speed $[3,51]$. This trend may be attributed to researchers and practitioners implementing tests that align with the testing protocols adopted by the NBA Draft Combine. Agility tests were the least reported category of test in the literature, and despite all studies assessing agility using a similar Y-Shape design the distances covered and stimuli used varied. Strength tests primarily incorporated bench press and back squat 1RM protocols. The frequency of strength testing observations was less than jump, linear sprint, change-of-direction speed, anaerobic capacity, and aerobic capacity tests, which may be due to varying levels of resistance training competency, biomechanical constraints introduced by the typically larger anthropometric values of basketball players, and the residual fatigue often accumulated by maximal strength testing. The anaerobic capacity of basketball players was tested predominantly 


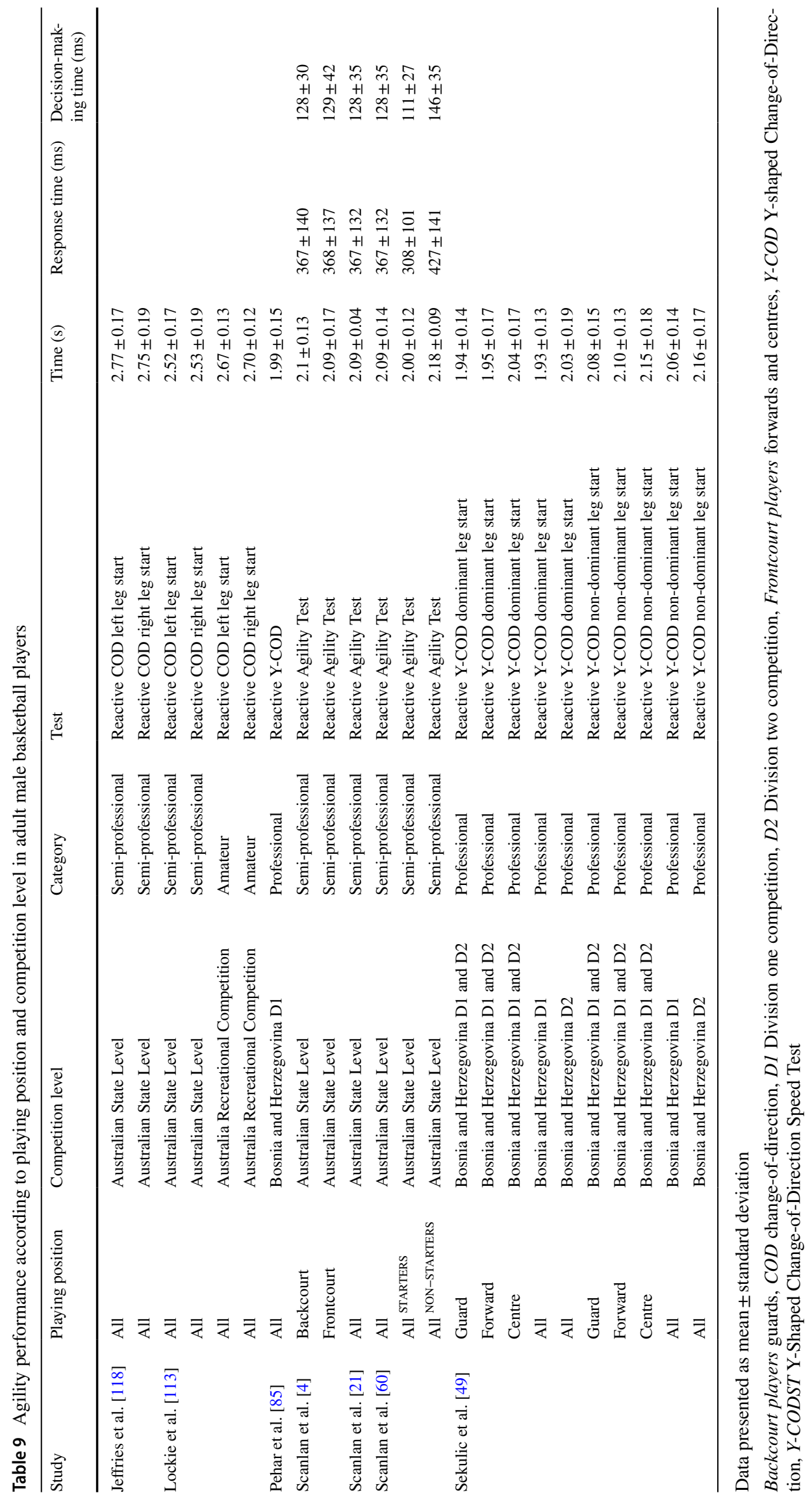




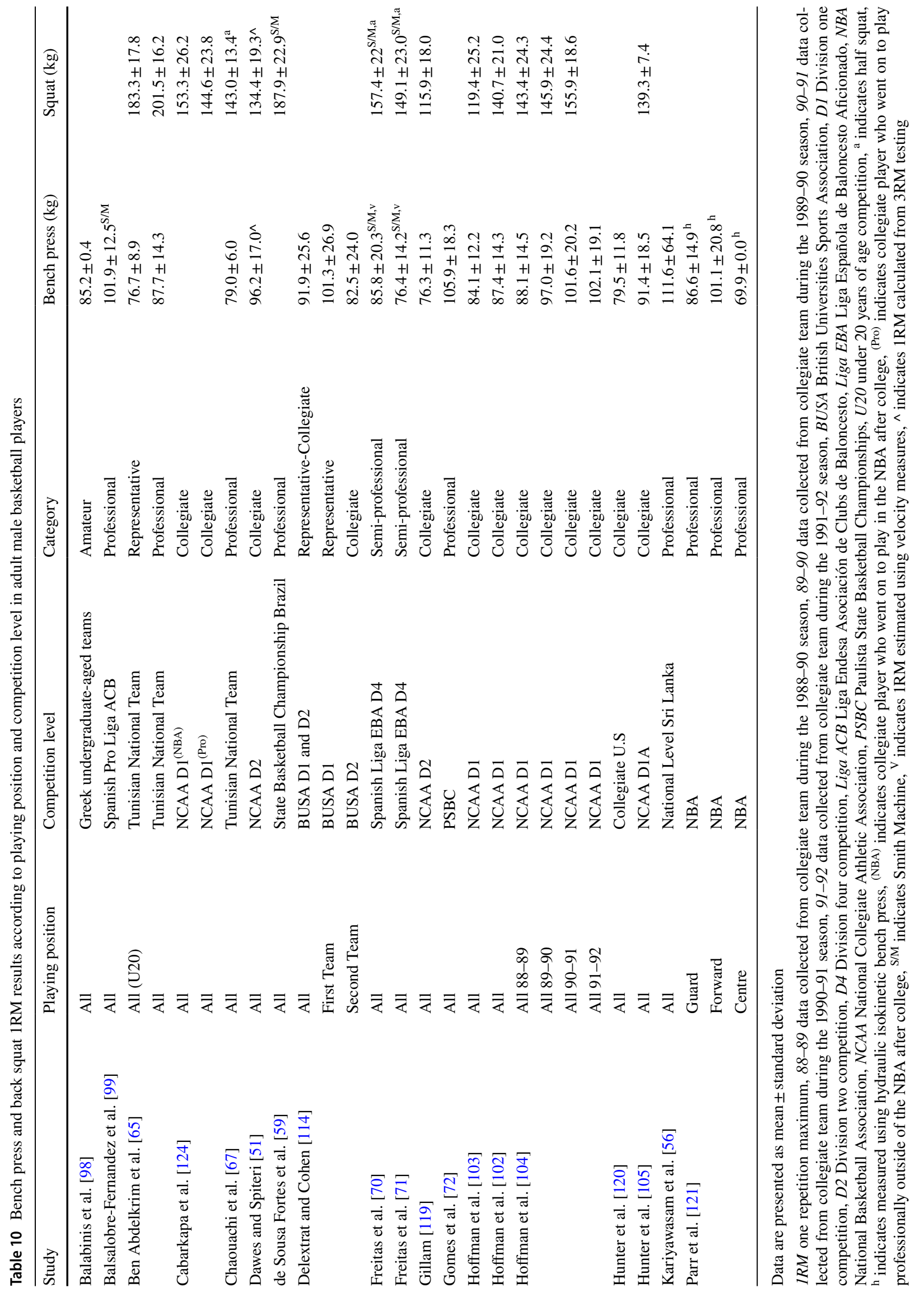


Table 11 Repeated sprint, running-based anaerobic sprint test and Wingate anaerobic cycle test performance according to playing position and competition level in adult male basketball players

\begin{tabular}{|c|c|c|c|c|c|c|}
\hline Study & Playing position & Competition level & Category & Test & Variable & Outcome \\
\hline Dawes and Spiteri [51] & All & NCAA D2 & Collegiate & Full court shuttle run & Time (s) & $27.8 \pm 0.9$ \\
\hline \multirow[t]{2}{*}{ Fatouros et al. [125] } & All & Greek D2 Competition & Professional & Full court shuttle run & Time (s) & $27.4 \pm 0.7$ \\
\hline & All & Greek Recreational & Amateur & Full court shuttle run & Time (s) & $29.2 \pm 0.9$ \\
\hline Maggioni et al. [58] & All & Volunteer Players & Semi-professional & Full court shuttle run & Time (s) & $27.8 \pm 0.8$ \\
\hline \multirow[t]{4}{*}{ Montgomery et al. [28] } & All & Australian State Level & Semi-professional & Full court shuttle run & Time (s) & $27.5 \pm 1.2$ \\
\hline & Guard & Australian State Level & Semi-professional & Full court shuttle run & Time (s) & $26.9 \pm 0.9$ \\
\hline & Forward & Australian State Level & Semi-professional & Full court shuttle run & Time (s) & $27.9 \pm 1.4$ \\
\hline & Centre & Australian State Level & Semi-professional & Full court shuttle run & Time (s) & $27.6 \pm 1.4$ \\
\hline \multirow[t]{3}{*}{ Pojskić et al. [87] } & All & Bosnian Premier League & Professional & RAST & Maximal power $(\mathrm{W})$ & $761 \pm 125$ \\
\hline & & & & & Mean power $(\mathrm{W})$ & $620 \pm 99.4$ \\
\hline & & & & & Fatigue index (W/s) & $8.2 \pm 1.4$ \\
\hline \multirow[t]{9}{*}{ Pojskić et al. [88] } & Guard & Bosnian Premier League & Professional & RAST & Maximal power $(\mathrm{W})$ & $773 \pm 129$ \\
\hline & & & & & Mean power $(\mathrm{W})$ & $635 \pm 110$ \\
\hline & & & & & Fatigue index (W/s) & $8.1 \pm 2.5$ \\
\hline & Forward & Bosnian Premier League & Professional & RAST & Maximal power $(\mathrm{W})$ & $762 \pm 123$ \\
\hline & & & & & Mean power $(\mathrm{W})$ & $608 \pm 89.6$ \\
\hline & & & & & Fatigue index (W/s) & $8.8 \pm 2.7$ \\
\hline & Centre & Bosnian Premier League & Professional & RAST & Maximal power $(\mathrm{W})$ & $858 \pm 109$ \\
\hline & & & & & Mean power $(\mathrm{W})$ & $713 \pm 69.5$ \\
\hline & & & & & Fatigue index (W/s) & $10.5 \pm 2.24$ \\
\hline \multirow[t]{3}{*}{ Pojskić et al. [89] } & All & Bosnian and Herzego- & Professional & RAST & Maximal power $(\mathrm{W})$ & $761 \pm 125$ \\
\hline & & vina D1 & & & Mean power $(\mathrm{W})$ & $620 \pm 99.4$ \\
\hline & & & & & Fatigue index (W/s) & $8.2 \pm 1.4$ \\
\hline \multirow{3}{*}{$\begin{array}{l}\text { Balsalobre-Fernandez } \\
\text { et al. [99] }\end{array}$} & All & Spanish Pro Liga ACB & Professional & RAST & Peak power $(\mathrm{W})$ & $957 \pm 194$ \\
\hline & & & & & Mean power $(\mathrm{W})$ & $772 \pm 126$ \\
\hline & & & & & Fatigue index $(\%)$ & $33.1 \pm 8.0$ \\
\hline \multirow{3}{*}{$\begin{array}{l}\text { Balsalobre-Fernandez } \\
\text { et al. [100] }\end{array}$} & All & Spanish Pro Liga ACB & Professional & RAST & Peak power $(\mathrm{W})$ & $889 \pm 230^{\mathrm{WPD}}$ \\
\hline & & & & & Mean power $(\mathrm{W})$ & $737 \pm 134^{\text {WPD }}$ \\
\hline & & & & & Fatigue index $(\%)$ & $32.4 \pm 7.2^{\text {WPD }}$ \\
\hline \multirow[t]{12}{*}{ de Araujo et al. [129] } & All & Elite National Team and & Professional & RAST & Peak power $(\mathrm{W})$ & $901 \pm 39.1$ \\
\hline & & League Brazıl & & & Mean power $(\mathrm{W})$ & $701 \pm 22.7$ \\
\hline & & & & & Fatigue index (\%) & $41.5 \pm 2.5$ \\
\hline & Guard & $\begin{array}{l}\text { Elite National Team and } \\
\text { League Brazil }\end{array}$ & Professional & RAST & Peak power $(\mathrm{W})$ & $853 \pm 59.0$ \\
\hline & & & & & Mean power $(\mathrm{W})$ & $683 \pm 49.0$ \\
\hline & & & & & Fatigue index (\%) & $42.1 \pm 4.5$ \\
\hline & Forward & $\begin{array}{l}\text { Elite National Team and } \\
\text { League Brazil }\end{array}$ & Professional & RAST & Peak power $(\mathrm{W})$ & $923 \pm 79.8$ \\
\hline & & & & & Mean power $(\mathrm{W})$ & $700 \pm 40.7$ \\
\hline & & & & & Fatigue index (\%) & $40.6 \pm 4.4$ \\
\hline & Centre & $\begin{array}{l}\text { Elite National Team and } \\
\text { League Brazil }\end{array}$ & Professional & RAST & Peak power $(\mathrm{W})$ & $912 \pm 42.0$ \\
\hline & & & & & Mean power $(\mathrm{W})$ & $717 \pm 26.4$ \\
\hline & & & & & Fatigue index $(\%)$ & $42.1 \pm 4.3$ \\
\hline Alemdaroglu [61] & All & Turkish D1 & Professional & Wingate Anaerobic Cycle Test & $\begin{array}{l}\text { Peak power }(\mathrm{W}) \\
\text { Mean power }(\mathrm{W}) \\
\text { Fatigue index }(\%)\end{array}$ & $\begin{array}{l}955 \pm 118 \\
703 \pm 79.3 \\
54.7 \pm 7.3\end{array}$ \\
\hline Balabinis et al. [98] & All & $\begin{array}{l}\text { Undergraduate Teams } \\
\text { Greece }\end{array}$ & Amateur & Wingate Anaerobic Cycle Test & Peak power $(\mathrm{W})$ & $841 \pm 92.4$ \\
\hline \multirow{6}{*}{$\begin{array}{l}\text { Delextrat and Cohen } \\
\text { [114] }\end{array}$} & All & BUSA D1 & Representative & Wingate Anaerobic Cycle Test & Peak power $(\mathrm{W} / \mathrm{kg})$ & $10.2 \pm 0.9^{*}$ \\
\hline & & & & & Mean power $(\mathrm{W} / \mathrm{kg})$ & $8.2 \pm 0.9^{*}$ \\
\hline & & & & & Fatigue index $(\%)$ & $57.4 \pm 14.9$ \\
\hline & All & BUSA D3 & Collegiate & Wingate Anaerobic Cycle Test & Peak power $(\mathrm{W} / \mathrm{kg})$ & $10 \pm 0.9^{*}$ \\
\hline & & & & & Mean power $(\mathrm{W} / \mathrm{kg})$ & $7.8 \pm 1.1^{*}$ \\
\hline & & & & & Fatigue index (\%) & $47.2 \pm 15.1$ \\
\hline
\end{tabular}


Table 11 (continued)

\begin{tabular}{|c|c|c|c|c|c|c|}
\hline Study & Playing position & Competition level & Category & Test & Variable & Outcome \\
\hline \multirow[t]{4}{*}{ Fatouras et al. [125] } & \multirow[t]{2}{*}{ All } & \multirow[t]{2}{*}{ Greek D2 Competition } & \multirow[t]{2}{*}{ Professional } & \multirow[t]{2}{*}{ Wingate Anaerobic Cycle Test } & Peak power $(\mathrm{W} / \mathrm{kg})$ & $11.2 \pm 2.1$ \\
\hline & & & & & Mean power $(\mathrm{W} / \mathrm{kg})$ & $9.3 \pm 0.9$ \\
\hline & \multirow[t]{2}{*}{ All } & \multirow[t]{2}{*}{ Greek Recreational } & \multirow[t]{2}{*}{ Amateur } & \multirow[t]{2}{*}{ Wingate Anaerobic Cycle Test } & Peak power (W/kg) & $9.7 \pm 1.9$ \\
\hline & & & & & Mean power $(\mathrm{W} / \mathrm{kg})$ & $7.2 \pm 2.1$ \\
\hline \multirow[t]{5}{*}{ Harbili [128] } & \multirow[t]{5}{*}{ All } & \multirow{5}{*}{$\begin{array}{l}\text { Turkish National League } \\
\text { D3 }\end{array}$} & \multirow[t]{5}{*}{ Professional } & \multirow[t]{5}{*}{ Wingate Anaerobic Cycle Test } & Peak power $(\mathrm{W})$ & $951.6 \pm 86.9$ \\
\hline & & & & & Peak power (W/kg) & $10.3 \pm 1.4$ \\
\hline & & & & & Mean power $(\mathrm{W})$ & $683.7 \pm 40.5$ \\
\hline & & & & & Mean power $(\mathrm{W} / \mathrm{kg})$ & $7.4 \pm 0.8$ \\
\hline & & & & & Fatigue index $(\%)$ & $59.9 \pm 6.3$ \\
\hline \multirow[t]{15}{*}{ Nikolaidis et al. [109] } & \multirow[t]{5}{*}{ Guard } & \multirow[t]{5}{*}{ Italian First League } & \multirow[t]{5}{*}{ Professional } & \multirow[t]{5}{*}{ Wingate Anaerobic Cycle Test } & Peak power $(\mathrm{W})$ & $992 \pm 155$ \\
\hline & & & & & Peak power (W/kg) & $11.4 \pm 1.1$ \\
\hline & & & & & Mean power $(\mathrm{W})$ & $737 \pm 78$ \\
\hline & & & & & Mean power $(\mathrm{W} / \mathrm{kg})$ & $8.5 \pm 0.7$ \\
\hline & & & & & Fatigue index $(\%)$ & $47.5 \pm 6.6$ \\
\hline & \multirow[t]{5}{*}{ Forward } & \multirow[t]{5}{*}{ Italian First League } & \multirow[t]{5}{*}{ Professional } & \multirow[t]{5}{*}{ Wingate Anaerobic Cycle Test } & Peak power $(\mathrm{W})$ & $1052 \pm 93$ \\
\hline & & & & & Peak power (W/kg) & $11.1 \pm 1.1$ \\
\hline & & & & & Mean power $(\mathrm{W})$ & $823 \pm 94$ \\
\hline & & & & & Mean power $(\mathrm{W} / \mathrm{kg})$ & $8.7 \pm 1.1$ \\
\hline & & & & & Fatigue index $(\%)$ & $42.9 \pm 4.7$ \\
\hline & \multirow[t]{5}{*}{ Centre } & \multirow[t]{5}{*}{ Italian First League } & \multirow[t]{5}{*}{ Professional } & \multirow[t]{5}{*}{ Wingate Anaerobic Cycle Test } & Peak power $(\mathrm{W})$ & $1085 \pm 93$ \\
\hline & & & & & Peak power (W/kg) & $10 \pm 1.1$ \\
\hline & & & & & Mean power $(\mathrm{W})$ & $807 \pm 91$ \\
\hline & & & & & Mean power $(\mathrm{W} / \mathrm{kg})$ & $7.4 \pm 0.8$ \\
\hline & & & & & Fatigue index $(\%)$ & $44.4 \pm 5.5$ \\
\hline $\begin{array}{l}\text { Popadic Gacesa et al. } \\
\text { [127] }\end{array}$ & All & Elite Serbian Players & Professional & Wingate Anaerobic Cycle Test & $\begin{array}{l}\text { Peak power }(\mathrm{W}) \\
\text { Peak power }(\mathrm{W} / \mathrm{kg}) \\
\text { Mean power }(\mathrm{W}) \\
\text { Mean power }(\mathrm{W} / \mathrm{kg})\end{array}$ & $\begin{array}{l}1001 \pm 150 \\
10.7 \pm 1.7 \\
669 \pm 77.1 \\
7.2 \pm 1\end{array}$ \\
\hline Sallet et al. [126] & All & French D1 and D2 & Professional & Wingate Anaerobic Cycle Test & Peak power (W/kg) & $12.2 \pm 2.7$ \\
\hline & & & & & Fatigue index $(\%)$ & $58.9 \pm 13.5$ \\
\hline & Guards & French D1 and D2 & Professional & Wingate Anaerobic Cycle Test & $\begin{array}{l}\text { Peak power }(\mathrm{W} / \mathrm{kg}) \\
\text { Fatigue index }(\%)\end{array}$ & $\begin{array}{l}13.1 \pm 1.7 \\
63.8 \pm 14.7\end{array}$ \\
\hline & Forwards & French D1 and D2 & Professional & Wingate Anaerobic Cycle Test & Peak power $(\mathrm{W} / \mathrm{kg})$ & $12.7 \pm 3.5$ \\
\hline & & & & & Fatigue index (\%) & $58.1 \pm 9.3$ \\
\hline & Centres & French D1 and D2 & Professional & Wingate Anaerobic Cycle Test & Peak power $(\mathrm{W} / \mathrm{kg})$ & $11.1 \pm 2.1$ \\
\hline & & & & & Fatigue index (\%) & $56.3 \pm 15.7$ \\
\hline Soslu et al. [117] & All & $\begin{array}{l}\text { Turkish Professional } \\
\text { Competition }\end{array}$ & Professional & Wingate Anaerobic Cycle Test & $\begin{array}{l}\text { Peak power }(\mathrm{W}) \\
\text { Peak power }(\mathrm{W} / \mathrm{kg}) \\
\text { Mean power }(\mathrm{W}) \\
\text { Mean power }(\mathrm{W} / \mathrm{kg})\end{array}$ & $\begin{array}{l}849 \pm 128 \\
12.4 \pm 3.0 \\
681 \pm 86 \\
8.7 \pm 1.2\end{array}$ \\
\hline
\end{tabular}

Data are presented as mean \pm standard deviation

BUSA British Universities Sports Association, D1 Division one competition, D2 Division two competition, D3 Division three competition, Liga $A C B$ Liga Endesa Asociación de Clubs de Baloncesto, NCAA National Collegiate Athletic Association, RAST Running-based Anaerobic Sprint Test, ${ }^{\text {WPD }}$ indicates data retrieved using WebPlotDigitizer

with repeated sprint protocols varying in distance or a resisted cycling WAnT test. Aerobic capacity was most frequently assessed using running incremental treadmill tests or estimated using the Yo-Yo IRL1 and the MSFT. The incremental treadmill test was primarily reported at the professional level, which may suggest the resources required to undertake the incremental treadmill test may not be feasible to obtain and implement across all competition levels.

The wide variety of testing approaches reported across studies make it challenging to establish normative data or identify minimum thresholds for physical characteristics in adult male basketball players. Researchers and practitioners are encouraged to diligently consider the tests they select when assessing physical characteristics in players, as each 


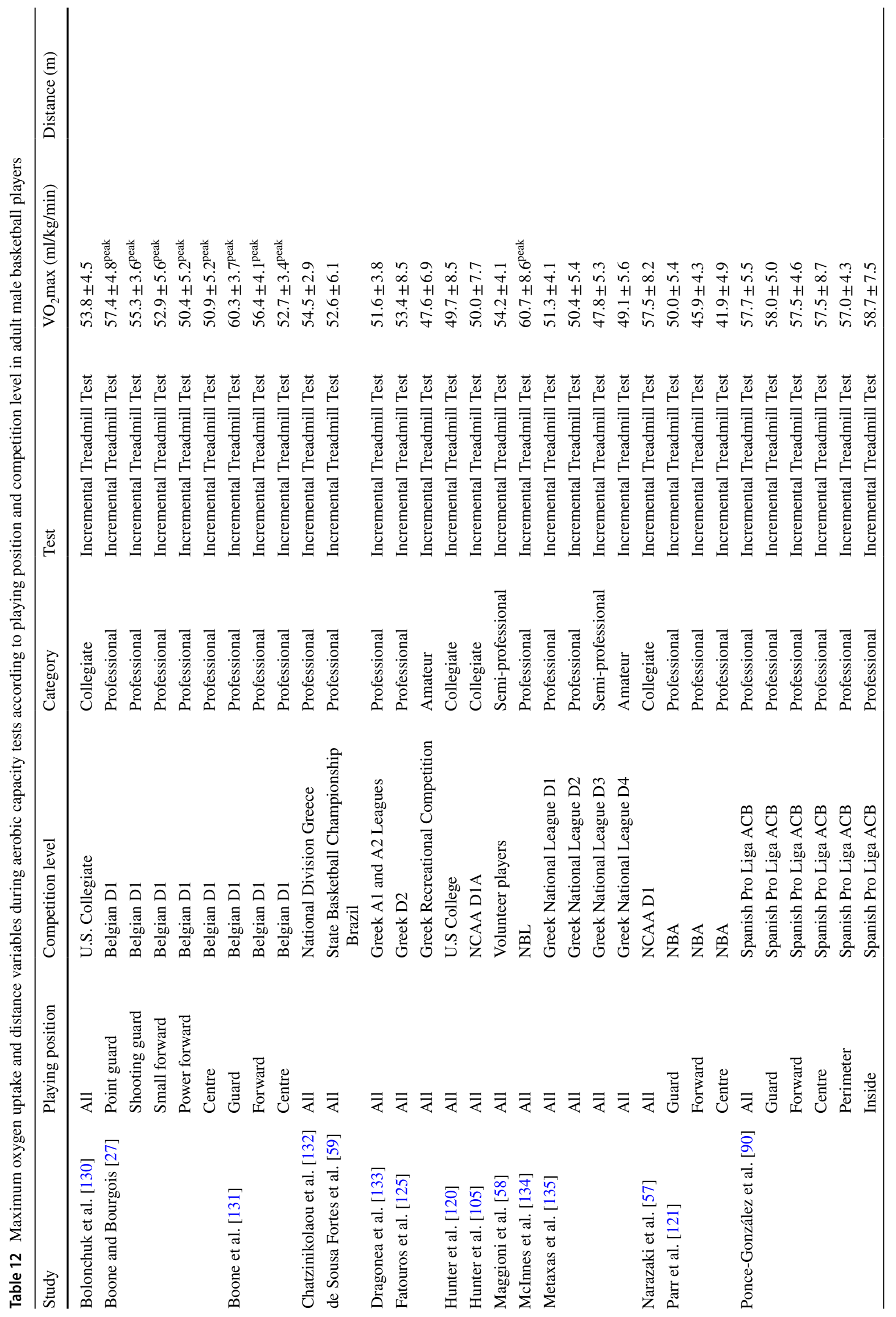




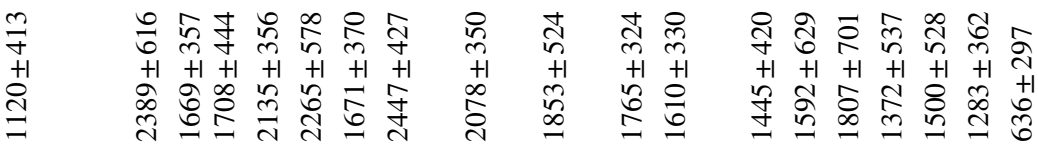

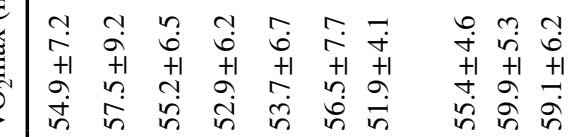

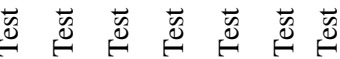

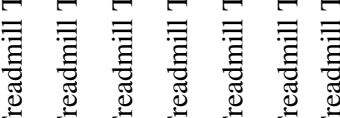

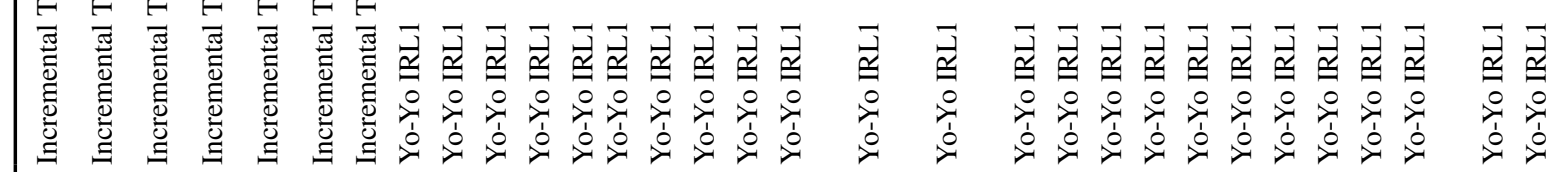

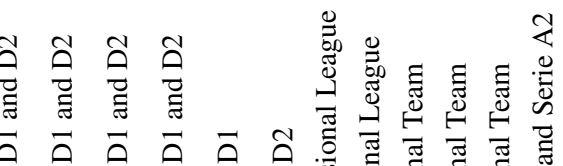
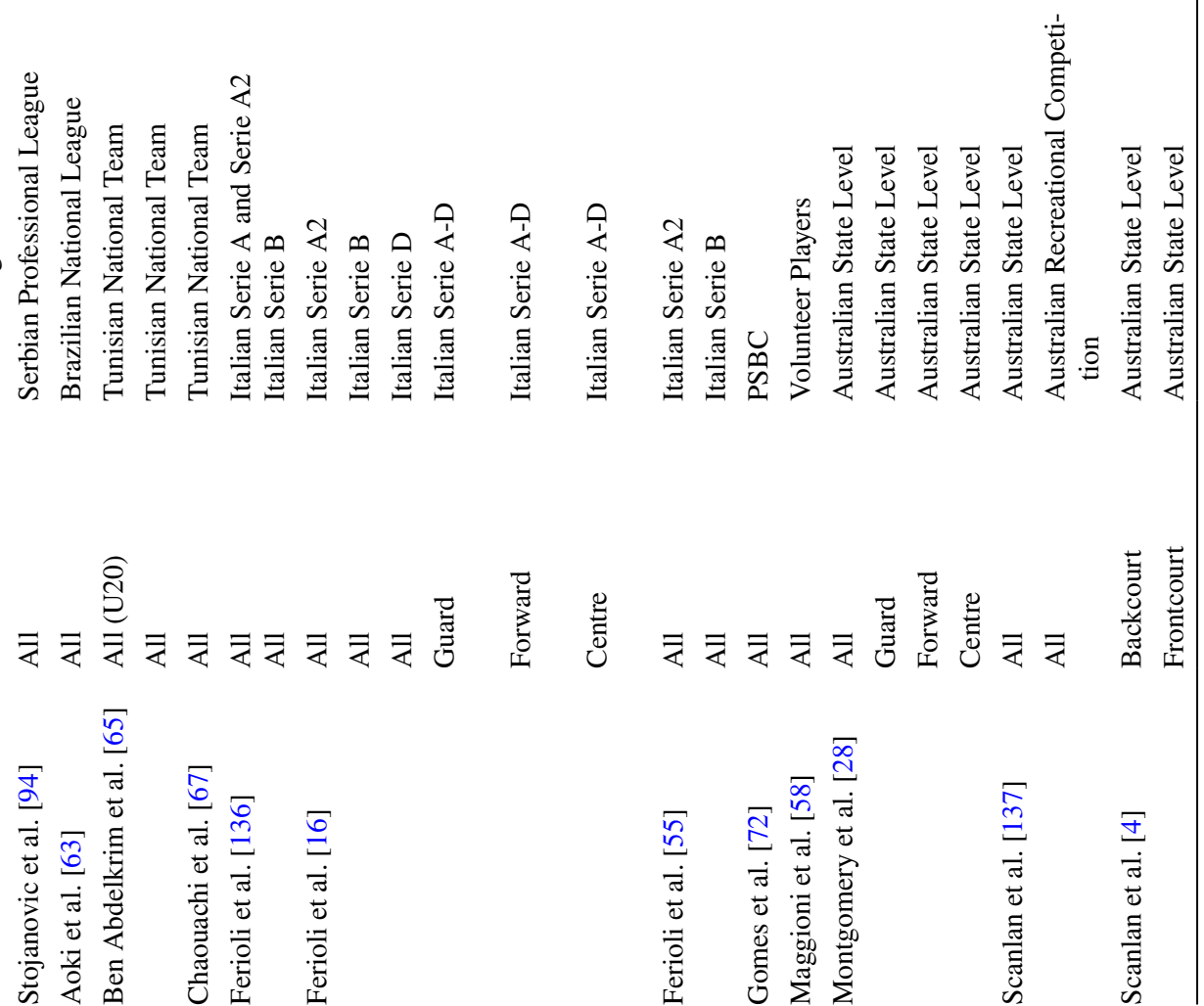

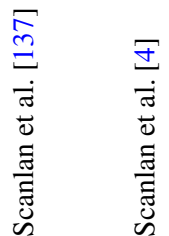




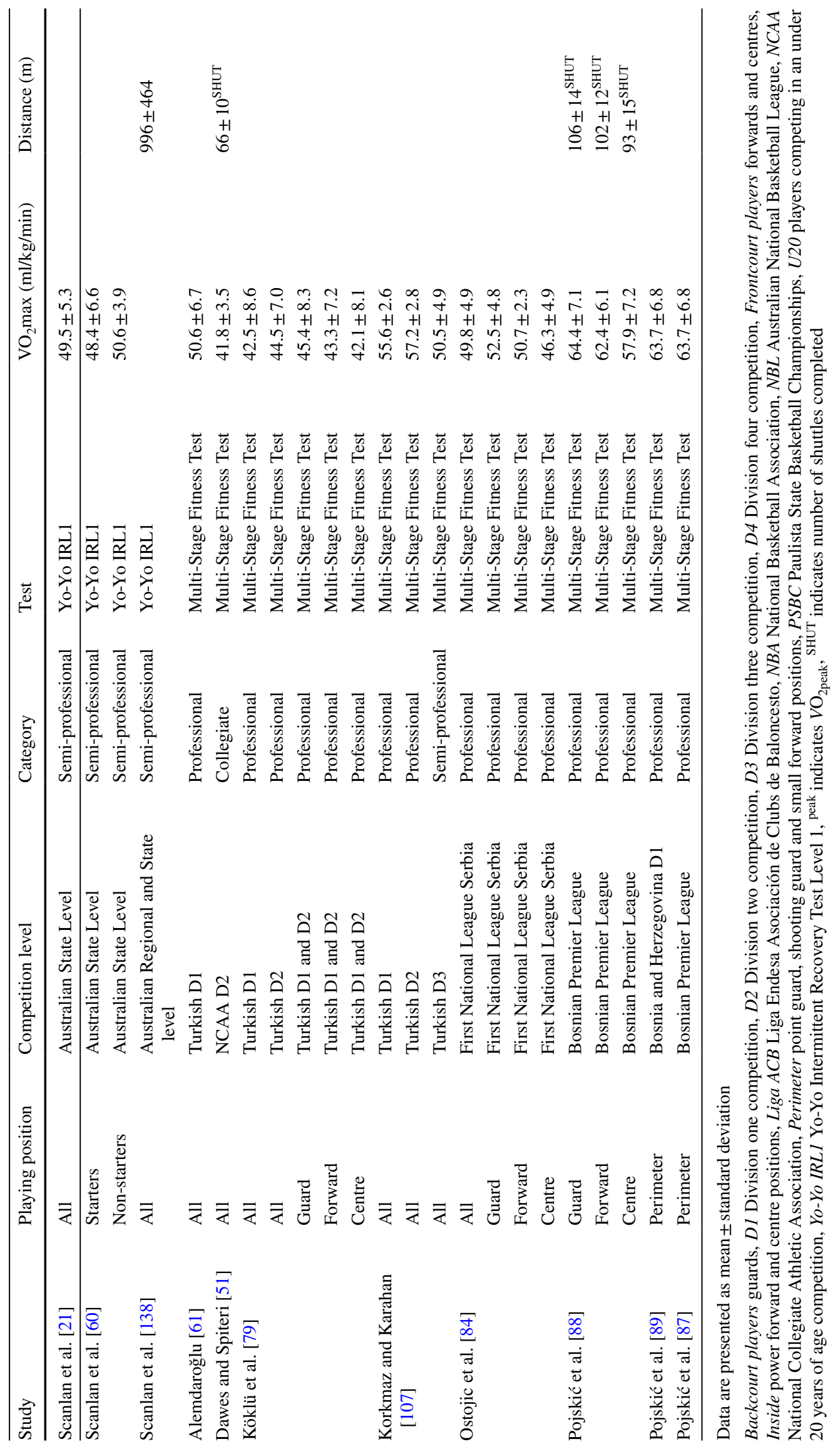


test and testing methodology has an inherent level of accuracy and reproducibility [140]. For example, when considering the methodology of jumping tests, a range of technology with varying levels of accuracy have been used to assess jump height, including force platforms [16, 61, 71, 110], three-dimensional cameras [110], contact mats $[62,83,96]$, Vertec [107], and chalk marks on a wall [103, 104]. The nuances associated with the various methodologies could influence results and need to be considered when comparing results between studies [140]. As consensus is reached with choice of test and methodology employed to measure key physical characteristics in adult male basketball players, it will become easier to monitor players and develop meaningful normative physical standards. In this regard, researchers and practitioners are encouraged to collaborate with FIBA and national governing bodies to develop standardised testing guidelines for broad application to basketball teams.

Basketball practitioners now have an abundance of published tests and methodologies to consider prior to assessing players. Additionally, there are outcome variables provided in multiple tests that represent different physical abilities. For example, jump height during a SJ provides insight into the ability to express concentric-only force, whereas jump height during a CMJ reflects the ability to use elastic energy that is generated during the countermovement [141]. Further, jump height from a running vertical jump is a measure of jumping performance specific to most common game situations [142]. Interpreting test results may be further complicated by multiple methods being available to calculate the same outcome variable. For instance, modified reactive strength index is commonly calculated as jump height divided by contraction time, yet can be calculated using jump height determined via flight time or impulse momentum [101]. This example highlights a major concern as variables such as flight time may be manipulated by a change in movement strategy by players (e.g., tucking legs on descent) subsequently altering results. Therefore, there are many aspects of current test selection that can be improved so all findings can contribute to establishing meaningful normative reference data.

Considering the wide variety of testing options adopted by basketball researchers and practitioners, relevance to the sport must be maintained and tests that do not directly transfer to basketball match-play should be carefully considered prior to implementation. When researchers and practitioners are selecting a test, they are encouraged to determine whether the bioenergetic and biomechanical components of a test are relevant and applicable to the needs of adult male basketball players. Furthermore, it is recommended that basketball researchers and practitioners consult with each other so that testing approaches can continue to be refined.

Basketball practitioners have several considerations and constraints at the organisational and team level that may influence selecting an appropriate testing battery. The accessibility of resources, technology, the expertise of staff on hand, availability of players, and influence of additional stakeholders can all influence the testing procedures adopted. Once tests are selected, an additional consideration when testing is the motivation of players. It is important to ensure players are executing testing with maximal intent when maximal efforts are required to avoid reporting submaximal performances. Providing the appropriate environment for testing is important as athletes are often tested for varying purposes (e.g., team selection, pre-season assessment) and at times, may lack incentive to give maximal effort. Basketball practitioners are encouraged to emphasise the importance of maximal effort during testing, as players who are fitter may appear as more resilient, effective and desirable for coaches. Despite these constraints, basketball practitioners are encouraged to select tests that are able to provide data that can allow for the monitoring of player progression, ranking or differentiation of players, and appropriate prescription of subsequent training. Additionally, basketball researchers and practitioners may wish to report test outcomes using ratios or indices (e.g., speed for height, agility to height ratio) that account for individual anthropometry as this may be beneficial when attempting to compare players who play across multiple positions. By scaling outcomes gathered from physical tests, performance may be able to be normalised and account for differences in player body size in sports where stature and mass are wide ranging [143].

\subsection{Physical Characteristics}

\subsubsection{Anthropometry}

Taller players with longer wingspans may have the ability to rebound the ball at greater heights, take up more space when defending an opponent, and more effectively contest shots. For a given speed of movement, skill set, and fitness level, basketball inherently favours taller players as reflected by anthropometry measures being the most frequently measured physical characteristics across studies in this review. When competition levels were grouped by mean team height, higher level players such as those competing professionally were taller than amateur players. However, the range of mean height observed across studies was similar for professional, semi-professional, representative, and collegiate adult male players. When height was reported relative to playing position, a clear trend emerged across competition levels with guards identified as the shortest players, forwards being taller than guards, and centres being the tallest players. Height and wingspan measured at the NBA Draft Combine have been identified as predictors of future playing performance in the NBA [144]. However, in many basketball 
studies, wingspan was scarcely reported $[3,51,52]$. To better understand the interaction of player height, wingspan and performance, basketball researchers and practitioners should measure and report wingspan alongside height and body mass as part of a standardised anthropometrical assessment.

Body mass followed a similar trend to height, with broad ranges observed at most competition levels. Mean body mass in semi-professional players exhibited the smallest range across studies of any competition level, possibly owing to the large representation in this review of players from Australian state-level competitions $[4,21,28,30,60,112,113$, $118,137,138,145]$. Furthermore, the body mass of players at higher competition levels tended to be heavier than players competing at lower levels likely because of players at higher levels possessing greater lean body mass and height. Positional differences in body mass were noted, with guards being lightest, forwards being heavier than guards, and centres heaviest $[27,49,129]$. This positional trend in body mass was apparent in players at the professional level; however, because of the lack of evidence available, positional differences in body mass at the semi-professional, representative, collegiate, and amateur levels are not yet clear.

\subsubsection{Body Composition}

Body composition was shown to differ between competition levels in adult male basketball players. In this regard, semiprofessional, representative, and collegiate players typically possessed a lower proportion of body fat than amateur players. Data retrieved from the NBA Draft Combine showed elite players drafted into the NBA typically exhibit very low levels of body fat. This finding may be because of higher level players having more availability for training and greater access to performance resources (e.g., dietitians and strength and conditioning coaches) or it may be the case that selectors for the NBA prefer lean players, as how lean a player is may have the potential to influence their match-play and ability to perform basketball skills. However, the comparison of body fat percentages using skinfold assessments across studies in this review must be made with caution as the anatomical landmarks used were not always consistent. For example, when the sum of three skinfolds was used to calculate body fat percentage, they were taken from the chest, abdomen, and thigh [53, 56, 57], the triceps, abdomen, and thigh $[4,21,60]$, and the triceps, chest, and subscapular [59] in different studies. A further consideration regarding body composition is often different techniques and equations can be used, leading to differences in body fat estimates. These variations in methodology may influence the results presented. When mean body fat percentage was reported according to playing position, similar ranges were observed between guards (7-20\%), forwards (8-17\%), and centres (7-21\%). However, the range of body fat of each position was noticeably influenced by one study [109], and when data from this study were excluded, values decreased across playing positions (guards: $7-14 \%$, forwards: $8-15 \%$, and centres: $7-16 \%$ ). These ranges may be a more appropriate summation of the body fat percentages in adult male players identified in this review and suggest body composition may be similar across playing positions.

A further consideration for basketball researchers and practitioners when interpreting or comparing results is the time of testing. For example, players may be assessed at the commencement of pre-season and record considerably different results compared with the middle of season, when they are likely insuperior physical condition. When measuring anthropometry, it is recommended basketball practitioners initiate their testing batteries with measures of height, body mass, body composition, and wingspan as these measurements may change after the initiation of other tests (e.g., fluid loss from an aerobic capacity test). Throughout this review, the phase of the basketball season and where testing occurred was often not reported. To improve future basketball research, it is strongly encouraged that when the testing occurs is clearly stated when reporting the physical characteristics of basketball athletes. Furthermore, when using sum of skinfolds to measure body composition, the specific sites should be clearly identified and used repeatedly over time for consistent measurements. The use of an International Society for the Advancement of Kinanthropometry-certified anthropometrist may also be of benefit for the accuracy and reproducibility of measurements [146]. It is recommended to use the sum of eight skinfold sites, measured at the biceps, triceps, subscapular, iliac crest, supraspinale, abdominal, anterior thigh and medial calf, in line with the recommended protocols outlined by the International Society for the Advancement of Kinanthropometry due to the efficiency of testing, ability to detect meaningful change over time, and standardisation of measurement [147].

\subsubsection{Muscular Power}

Well-developed muscular power is favourable to meet the physical demands of basketball match-play $[16,104,114$, 148]. Jump variables were used most frequently to indirectly assess muscular power characteristics, which may be due to the range of tasks specific to basketball involving various forms of jumps (e.g., rebounding, contesting a shot) and the high frequency of jumping executed throughout matches [12, $149,150]$. The greatest jump heights reported in the literature were from the $\mathrm{VJ}$ test in collegiate players $(77 \pm 6 \mathrm{~cm})$ [111] and players who were assessed at the NBA Draft Combine $(77 \pm 8 \mathrm{~cm})$. Collegiate players $(44-83 \mathrm{~cm})$ tended to record higher VJ performances than professional players $(36-63 \mathrm{~cm})$, and semi-professional players $(35-50 \mathrm{~cm})$, while insufficient studies were observed at the representative 
and amateur levels to draw conclusions. No differences in VJ height were observed between playing positions at the professional level with insufficient studies available across other playing levels to make conclusions regarding positional differences. Considering there were discrepancies between professional, semi-professional, and collegiate players observed, it is important to consider that different testing methods (e.g., three vs five jumps, mean jump height vs greatest jump height) may have been used across studies. Therefore, the influence of the different methods used to quantify jump height on the quality of the reported data is not known and requires further investigation.

Mean CMJ height ranged from 35 to $77 \mathrm{~cm}$ across studies in adult male basketball players. Multiple studies reported jump height and peak power of professional, semi-professional, and collegiate players. Findings demonstrated heterogenous outcomes for jump height and peak power output both within (e.g., professional vs professional) and between (e.g., professional vs collegiate) competition levels. The lack of research at the representative and amateur playing levels limited the ability to draw conclusions regarding CMJ performance for players competing at these levels. The variation in CMJ height and peak power output observed in professional, semi-professional, and collegiate players may be reflective of the different testing methodologies used across studies combined with the varying abilities of players assessed across different competition levels. When mean CMJ height was reported according to playing position irrespective of competition level, similar ranges were observed (guards: $38-60 \mathrm{~cm}$, forwards: $36-58 \mathrm{~cm}$, and centres: $36-57 \mathrm{~cm}$ ). From a methodological perspective, when conducting a $\mathrm{CMJ}$ on a force platform, basketball practitioners are able to record and track variables that are sensitive to changes over time (e.g., relative power output [151]), as well as monitor acute changes in jump performance (e.g., height) or strategy (e.g., flight time to contraction time ratio [152]). These varied options for interpretation may be of particular benefit when assessing and monitoring player fatigue and readiness across the season [153].

The SJ was also implemented to assess adult male basketball players throughout the literature but only in professional and collegiate players. Insufficient data were reported at the semi-professional, representative, collegiate, and amateur competition levels to draw conclusions. While professional player SJ height ranged $(29-50 \mathrm{~cm})$ across studies. Positional differences in SJ height were reported only at the professional level, with no clear differences apparent and centres exhibiting the least variability (guards: $30-41 \mathrm{~cm}$, forwards: $29-40 \mathrm{~cm}$, centres: $33-36 \mathrm{~cm}$ ). The jump height attained during the $\mathrm{SJ}$ was consistently lower than the CMJ and VJ. These differences are due to the concentric-only force expression of the SJ and the inability to use elastic energy generated during the preparatory countermovement evident in the CMJ and VJ [150]. However, when the SJ and $\mathrm{CMJ}$ tests are used together, the combination of outcome variables (e.g., jump height) can be used as a diagnostic tool, allowing basketball practitioners to evaluate the ability of players to use their stretch-shortening cycle while jumping [90]. However, identifying the reliability of these diagnostic variables such as the eccentric utilisation ratio, which can be calculated as CMJ height divided by SJ height (peak power may also be used), requires more research in basketball players.

The high frequency of jumps performed during basketball games has been well established [10, 12]. However, the quantity of different jump types (e.g., stationary jump vs running vertical jump, unilateral vs bilateral take-off), the number of maximal and submaximal jumps, and whether there are differences in the frequency of different jumps between playing positions or competition levels is unknown. This gap in the literature is a limitation when designing training programmes to enhance jump performance in basketball players, as the exact type of jumping demands imposed on players are not fully understood. While it is clear different jump strategies exist, it is important to recognise they are underpinned by different speed-strength qualities (e.g., reactive strength, concentric-only speed strength) [139]. Consequently, to holistically assess jumping ability in players, multiple tests may be required. A battery of tests that target a range of force production strategies such as SJ for concentric only force production, $\mathrm{CMJ}$ for long-slow stretch shortening cycle force production, and drop or repeated jumps for shortfast stretch shortening cycle production warrant consideration. Additionally, assessing jump performance from varying approaches and take-off strategies may provide further insight into jumping ability. Reporting arm reach during a jump, or the combination of jump height and wingspan may provide novel insight into the maximal ability of players to secure a rebound or tip the ball to advantage during matchplay. The combination of multiple jump tests may enable individual jump-profiles to be developed and allow training programmes to target the unique deficiencies of each player. However, it must be acknowledged that implementing an extensive jump battery may not be practical. Therefore, basketball practitioners may wish to select the most appropriate tests from the provided recommendations that best suits their needs.

Basketball players are frequently required to execute repeated jumps to challenge opposition shots and contest rebounds during match-play. While stationary bilateral jumps provide valuable information regarding the vertical jump ability of players, often multiple jumps are required in quick succession (e.g., multiple jumps while contesting a rebound). Therefore, an assessment of the speed-strength quality that underpins repeated jumping is warranted during testing. The reactive strength index represents reactive jump 
ability and has traditionally been assessed using drop jumps [154] or repeated jump protocols [96, 155] in male basketball players. However, we propose a novel bilateral hopping test protocol $[156,157]$ to measure both reactive strength and leg stiffness. While we are unaware of any basketball research that incorporates the bilateral hopping test, reactive strength index and leg stiffness are important qualities in basketball players because of the need to perform repeated jumps during training and matches. Furthermore, the bilateral hopping test has been shown to demonstrate greater between-day reliability compared with other repeated jump tests in adolescent rugby union players (e.g., five repeated jumps in place [157]). Additionally, the bilateral hopping test can be efficiently completed through a single test (compared with drop jump profiles that require multiple jumps) and can be standardised with the use of a metronome to ensure consistency.

\subsubsection{Linear Sprint Performance}

The game demands of basketball require well-developed linear sprint and acceleration capacities [12, 61, 65, 158-160]. Throughout the literature, heterogenous linear sprint distances have been used to assess adult male basketball players. When observing the three most commonly used distances, often insufficient data were observed to draw firm conclusions. Of the studies that reported linear sprint performance across distances up to $20 \mathrm{~m}$, only three [4, 21, 58] also reported 5-m and 10-m splits. Over $10 \mathrm{~m}$, the limited available evidence $[4,27,79]$ suggests guards $(1.72-2.19 \mathrm{~s})$ and forwards (1.72-2.25 s) possess similar linear sprint speed, and are faster than centres (1.80-2.34 s). Considering the match demands of basketball [7, 11, 12], researchers and practitioners are strongly encouraged to capture split times at the 5-m and 10-m marks during a 20-m linear sprint test. The additional data captured at $5 \mathrm{~m}$ and $10 \mathrm{~m}$ reflect distances that are encountered during match-play [7, 112] and may provide further insight into the acceleratory ability of players. Furthermore, basketball researchers and practitioners would benefit by reporting sprint times relative to playing position to help establish meaningful position-specific normative data that can assist with determining minimum thresholds for playing standards for adult male basketball players. Additionally, reporting sprint times relative to height may provide novel insight into the sprint capabilities of players and may be appropriate for categorising players who play across multiple positions throughout a match.

\subsubsection{Change-of-Direction Speed}

The ability to rapidly change direction within the confines of the court is important for basketball performance [20, 65, 161, 162]. Change-of-direction speed was most commonly assessed using the Agility $T$-Test. Observations between competition levels suggest change-of-direction speeds are similar between professional (8.84-10.04 s) and collegiate (8.92-9.78 s) players, with slower times evident in semiprofessional players $(9.52-10.90 \mathrm{~s})$. There were insufficient data to draw conclusions regarding positional differences in Agility $T$-Test performance. Further research is recommended to explore whether differences in change-of-direction speed are apparent between playing positions at other competition levels. However, the Agility $T$-Test has been scrutinised as it has been shown to favour specific physical characteristics such as 10-m linear sprint speed $(r=-0.92)$ and shuffling speed to the right $(r=-0.75)$ in semi-professional male basketball players [112]. A further concern of the Agility $T$-Test is the distances covered are not reflective of match requirements in basketball [12,112]. Consequently, a proposed modified Agility $T$-Test, where the distances are shortened to better reflect match demands players encounter has been suggested as an alternative option to assess changeof-direction speed in basketball players [148]. Nevertheless, this test has only been reported in one study examining adult male basketball players [64], and the validity and utility of a modified Agility $T$-Test as a measure of change-of-direction speed in adult male basketball players is not yet known and warrants further research.

Alternative change-of-direction speed tests such as the Y-Shaped Change-of-Direction Speed Test have also been used to assess adult male basketball players, but only in semi-professional players. Consequently, firm conclusions regarding the efficacy of the Y-Shaped Change-of-Direction Speed Test to discriminate between playing positions and competition levels is unclear. Moreover, a concern of the Y-Shaped Change-of Direction Speed Test is the lack of lateral movements, which are regularly performed in basketball match-play. In recent years, the Lane Agility Test has been used to assess change-of-direction speed at the collegiate level in adult male basketball players [3, 51, 111]. Similar to the Agility $T$-Test, the requirements of the Lane Agility Test are not reflective of most movement tasks commonly required during basketball match-play (i.e., no cognitive or perceptual elements present). Nonetheless, the Lane Agility Test consists of pre-determined periods of accelerating, lateral shuffling, and backwards running, all of which are typical movements in basketball. Mean Lane Agility Test performance was similar between collegiate players (10.4-11.8 s) and data captured at the NBA Draft Combine (10.3-12.2 s). Considering the collegiate playing pathway is a common route to playing professionally in the NBA, basketball researchers and practitioners may benefit from implementing the Lane Agility Test to their testing batteries to familiarise their players with the demands of the NBA Draft Combine if their players intend on entering the NBA draft. However, further exploration to identify the ability of 
the Lane Agility Test to discriminate change-of-direction speed between playing positions and competition levels is required. These findings suggest change-of-direction speed alone may not yet provide basketball researchers and practitioners with sufficient information to confidently evaluate and discriminate between adult male players competing at different levels.

\subsubsection{Agility}

Basketball match-play requires players to interpret stimuli and rapidly execute an appropriate movement response $[20,149]$, highlighting the need for a perceptual element to be present when assessing agility [47]. However, physical and technical components such as lower-body strength and movement strategy also contribute to agility performance $[46,47]$. The introduction of a decision-making constraint has indicated some agility tests are better able to discriminate between competition levels (semi-professional vs amateur [113]) and playing roles (starters vs non-starters [60]) compared with pre-planned change-of-direction tests in adult male basketball players. Consequently, the perceptual component present during an agility test may be of greater importance in discriminating between players at different competition levels than pre-planned change-of-direction speed. However, it is important to acknowledge that changeof-direction speed and agility are independent skills [46]. Considering the limited amount of research investigating agility in basketball players, further research is needed to explore potential differences between playing positions and competition levels in adult male basketball players and to develop an efficacious and ecologically valid agility test.

Finally, if implementing an agility test in basketball players, the type of stimuli being used should be considered. In football codes, a sport-specific stimulus has been shown to be an important component when assessing agility [163] with players competing at higher levels often performing better than players competing at lower levels in Australian rules football $[164,165]$ and rugby league $[166,167]$. Throughout the literature, timing light systems [113, 118], light-up cone systems $[49,85]$, and humans who initiate movement $[4,21,60]$ were the stimuli identified in agility tests used to assess adult male basketball players. Across studies included in this review, basketball researchers and practitioners emphasised accuracy during trials (i.e., the participant made the correct decision) as 'important' $[4,60]$ as identifying and executing the appropriate movement strategy during match-play may lead to better outcomes (e.g., anticipating the opponents movement and drawing an offensive foul) than if the incorrect decision was made (e.g., being called for a blocking foul due to being out of position rather than successfully drawing an offensive foul). However, the accuracy of the attempts were not always reported [4, 21, $49,60,85,113,118]$. Often if players made the incorrect decision or anticipated the required movement rather than responding to the stimuli during a trial, the attempt was discarded, not included in the data reported, and repeated [113, 118]. Therefore, it is recommended basketball researchers and practitioners report the outcome of agility trials (i.e., successful or unsuccessful) in the future as this may provide greater insight to the decision-making ability of players. The development of an outcome-based assessment of attacking and defending agility in basketball may provide a more comprehensive assessment of agility in adult male basketball players.

\subsubsection{Strength}

Muscular strength is an important quality in basketball players [94, 124, 168-170]. The limited data pertaining to lower-body strength across competition levels suggest professional players (back squat 1RM: $143-202 \mathrm{~kg}$ ) possess greater lower-body strength than collegiate players (back squat 1RM: 116-156 kg). In collegiate players, lower-body strength has been related to career obtainment, with stronger players reaching higher competition levels than their weaker peers [124]. Additionally, back squat 1RM has been shown to have a strong correlation with playing time in NCAA division $1(r=0.64)$ and division $2(r=0.74)$ competitions [51, 104]. The limited evidence available inhibits the ability to discern if lower-body strength can discriminate between playing positions in adult male basketball players. Consequently, the current positional demands and minimum physical thresholds of lower-body strength required by each playing position and competition level are unknown in adult male basketball. To address these gaps, basketball researchers and practitioners are encouraged to report strength testing results according to playing position to elucidate any positional differences that may be present.

Upper-body strength is often required in basketball matches when players compete to create and defend space. However, it is important to acknowledge that in order for isolated upper-body strength to transfer to match-play, contributions from force production components of the lower body may be required. The range of $1 \mathrm{RM}$ loads lifted during the bench press were similar between professional $(70-112 \mathrm{~kg})$ and collegiate $(76-102 \mathrm{~kg}$ ) players, with insufficient data reported for other competition levels. The lack of upperbody strength data observed for players competing at lower competition levels could be attributed to strength testing not being prioritised by basketball researchers and practitioners due to the fatigue induced from testing and level of exercise competency required of players. A possible solution for basketball researchers and practitioners to gather strength data from their players is to use a linear position transducer (LPT) 
during resistance training sessions to measure kinetic and kinematic outputs $[36,171]$. The use of an LPT during strength training can provide valid and reliable performance data [172], which is able to be tracked over time to monitor player progression (e.g., changes in bar speed at a specified load) $[171,173]$, and used to predict maximal strength whilst inducing minimal fatigue [171, 174, 175]. Only one study [121] reported bench press 1RM relative to playing position in adult male basketball players. Consequently, further research is required to confidently establish the upper-body strength characteristics of each playing position.

The evidence collated in this review indicate that strength testing in adult male basketball players requires further research to fully understand the minimum strength standards required of each playing position and competition level. Nonetheless, it is recommended basketball researchers and practitioners continue testing maximal upper-body and lower-body strength to monitor changes in strength across time. Additionally, the assessment of maximal strength will allow for accurate prescription of resistance training loads (e.g., \%1RM). Furthermore, the combination of dynamic and isometric strength tests may allow basketball researchers and practitioners to profile temporal and absolute force production ability in players. While there are numerous isometric tests available to assess force production characteristics, such as the isometric squat and isometric mid-thigh pull (IMTP), there is little published evidence exploring their utility and efficacy in basketball. The IMTP is an isometric test proposed to measure strength and force production characteristics of basketball players, owing to the ease of use and minimal fatigue induced in players [148]. The IMTP may also provide basketball researchers and practitioners with an option to test players who do not have the competency to undertake maximal dynamic strength testing. Furthermore, highly sensitive variables (e.g., early-phase force development across time bands) measured during the IMTP may be used to monitor player fatigue [176]. Therefore, a strength profile comprising the IMTP, bench press, and back squat may allow basketball researchers and practitioners to develop baseline levels of strength that support training prescription and are also able to guide return to play from injury.

\subsubsection{Anaerobic Capacity}

Well-developed anaerobic capacity allows basketball players to repeatedly perform high-intensity movements that are typically separated by brief rest periods during matches [17, 88, 177]. Assessment of anaerobic capacity involved either running or resisted cycling tests. Regarding running tests, full court shuttle run performance was homogenous (27.4-27.8 s) across professional [125], semi-professional [28], and collegiate [51] adult male basketball players.
Additional data are needed to determine if positional differences in the full court shuttle run exist and if the test is able to confidently discriminate between competition levels. The RAST was also used to assess anaerobic capacity exclusively in professional players, prohibiting the ability to compare performance between competition levels. Insufficient studies were observed to draw conclusions regarding positional differences in RAST performance in adult male basketball players. Subsequently, further research is required to elucidate any differences in RAST performance by playing position or competition level.

Regarding cycling tests, the WAnT cycle test was primarily used to assess anaerobic capacity and only reported in professional players. This isolated use of the WAnT cycle test in professional teams may be due to higher level organisations having greater access to specialised equipment and expertise to reliably implement this test. Additionally, the time associated with testing players using the WAnT cycle test may be impractical at lower competition levels. Insufficient data were available to draw conclusions regarding positional performance during the WAnT. While the data gathered from the WAnT cycle test provide valuable insight regarding the anaerobic capacity of basketball players, the time and resources required to implement the WAnT are considerable. Furthermore, the transfer of cycling anaerobic power to relevant sustained high-intensity movement patterns in basketball are not known. Therefore, basketball researchers and practitioners are encouraged to continue assessing the anaerobic capacity of adult male basketball players using tests that are accessible and appropriate to their needs. Additionally, reporting outcome variables indicative of anaerobic capacity relative to playing position using data in absolute terms and relative to body mass is recommended.

\subsubsection{Aerobic Capacity}

Basketball players require well-developed aerobic capacities to tolerate the intermittent bouts of varying intensity encountered during matches [129, 155, 178-181]. Players with a high aerobic capacity are better able to tolerate multiple high-intensity sprints and have improved fatigue resistance [182]. Throughout the literature, mean estimated and measured $\mathrm{VO}_{2 \max }$ ranged from 42 to $64 \mathrm{~mL} / \mathrm{kg} / \mathrm{min}$ across studies in adult male basketball players. This variation in results may be attributed to different tests being adopted (e.g., MSFT vs Yo-Yo IRL1 vs incremental treadmill test) and the inherent levels of error when calculating $\mathrm{VO}_{2 \max }$ during each test [183].

The use of incremental treadmill tests was evident at professional (50-61 mL/kg/min) and collegiate $(50-58 \mathrm{~mL} / \mathrm{kg} /$ min) levels and revealed similar well-developed mean aerobic capacity across both competition levels. Insufficient data were observed at the semi-professional, representative, and 
amateur levels to draw conclusions regarding the incremental treadmill test. Positional comparisons indicated guards possess the greatest mean aerobic capacity across studies in professional players $(50-60 \mathrm{~mL} / \mathrm{kg} / \mathrm{min})$, then forwards (46-58 $\mathrm{mL} / \mathrm{kg} / \mathrm{min})$, followed by centres $(42-58 \mathrm{~mL} / \mathrm{kg} /$ $\mathrm{min})$. The positional differences in aerobic capacity may be attributed to the unique match roles required of each position. The frequent use of incremental treadmill tests to assess $\mathrm{VO}_{2 \max }$ in professional and collegiate players may be attributed to basketball practitioners at these levels having greater access to resources such as laboratory-based physiological testing equipment than lower levels. Furthermore, professional and collegiate players may have a greater availability for testing throughout the year compared with other competition levels (e.g., semi-professional players may have competing demands such as supplementary jobs).

In a practical setting, the ability to test players efficiently is an important consideration for basketball researchers and practitioners, and the ability to test multiple athletes simultaneously is often advantageous. A variety of runningbased tests that are able to assess multiple players at once were identified in the literature. The two most commonly used tests were the Yo-Yo IRL1 and the MSFT, with the Yo-Yo IRL1 used most frequently at the professional and semi-professional levels. In contrast, the MSFT was used mainly in professional players. Positional differences in $\mathrm{VO}_{2 \max }$ attained during the MSFT reflect a similar trend to aerobic capacity from incremental treadmill tests, with professional guards recording the greatest estimated $\mathrm{VO}_{2 \max }$ (45-64 $\mathrm{mL} / \mathrm{kg} / \mathrm{min})$, then forwards $(43-62 \mathrm{~mL} / \mathrm{kg} / \mathrm{min})$, and centres $(42-58 \mathrm{~mL} / \mathrm{kg} / \mathrm{min})$. However, insufficient data were available to compare MSFT performance across competition levels and to identify differences in $\mathrm{VO}_{2 \max }$ between playing positions using the Yo-Yo IRL1. Therefore, further research is required to contribute meaningful data for the development of normative standards regarding the aerobic capacity requirements according to playing position and competition level in adult male basketball players.

\section{Limitations}

While this review presents a contemporary and comprehensive analysis of basketball tests and reveals the physical characteristics of adult male basketball players, there are limitations that should be considered. First, this review excluded tests involving a basketball-specific skill component (e.g., dribbling or shooting) and tests that assessed several physical characteristics simultaneously (e.g., Basketball Exercise Simulation Test [137, 138]). While these tests may offer novel insight regarding basketballrelated fitness, they are often assessing multiple physical characteristics at the same time, and therefore were not considered in this review. Second, the interaction between physical characteristics, psychological influences, technical abilities, tactical abilities, and the competitive environment in relation to basketball performance was not investigated. Thus, discriminating between competition levels or selecting players principally based on their physical characteristics is cautioned, as enhanced physical characteristics alone do not guarantee that a player will be successful. Furthermore, it is important to acknowledge that varying levels of inherent natural ability, underpinned largely by genetic components exist. However, there are elements of fitness such as fatigue resistance and muscular endurance that are able to be enhanced by appropriate training. Finally, due to the heterogeneity of testing methods reported in the literature, we were unable to perform a meta-analysis of physical characteristics across playing positions and competition levels.

\section{Practical Recommendations and Considerations for Testing Physical Characteristics}

With the wide range of tests and outcome variables available to basketball researchers and practitioners, developing a testing battery that is both valid and reliable but also informative and efficient can be challenging and at times contentious. Establishing a universal standardised test battery is further complicated by constraints such as resource availability, access to players, and competition or travel schedules that may interfere with scheduling testing sessions. While acknowledging these challenges, testing recommendations and outcome variables for each physical characteristic have been provided (Fig. 5 and Table 13). The proposed battery aims to allow for the standardisation of testing and the implementation of a reproducible protocol that can be used to inform subsequent training practice. Furthermore, the recommended tests are selected based on their efficiency (i.e., ability to test multiple players simultaneously or in succession) and the ability to use variables from multiple tests to infer additional qualities (e.g., sprint momentum, eccentric utilisation ratio). Finally, the testing battery is aimed to be applicable for the real-world assessment of adult male basketball players while drawing upon the scientific literature. It should be noted that this battery is not an exhaustive list of tests and outcome variables, and basketball researchers and practitioners are recommended to add or remove tests and output variables as they see fit provided their decisions are guided by logic, rationale, and data.

It is undeniable that anthropometry is an important consideration for basketball players [10, 32, 33, 184-192]. Thus, the measurement of height, body mass, and wingspan are strongly recommended at the beginning of testing. Following 


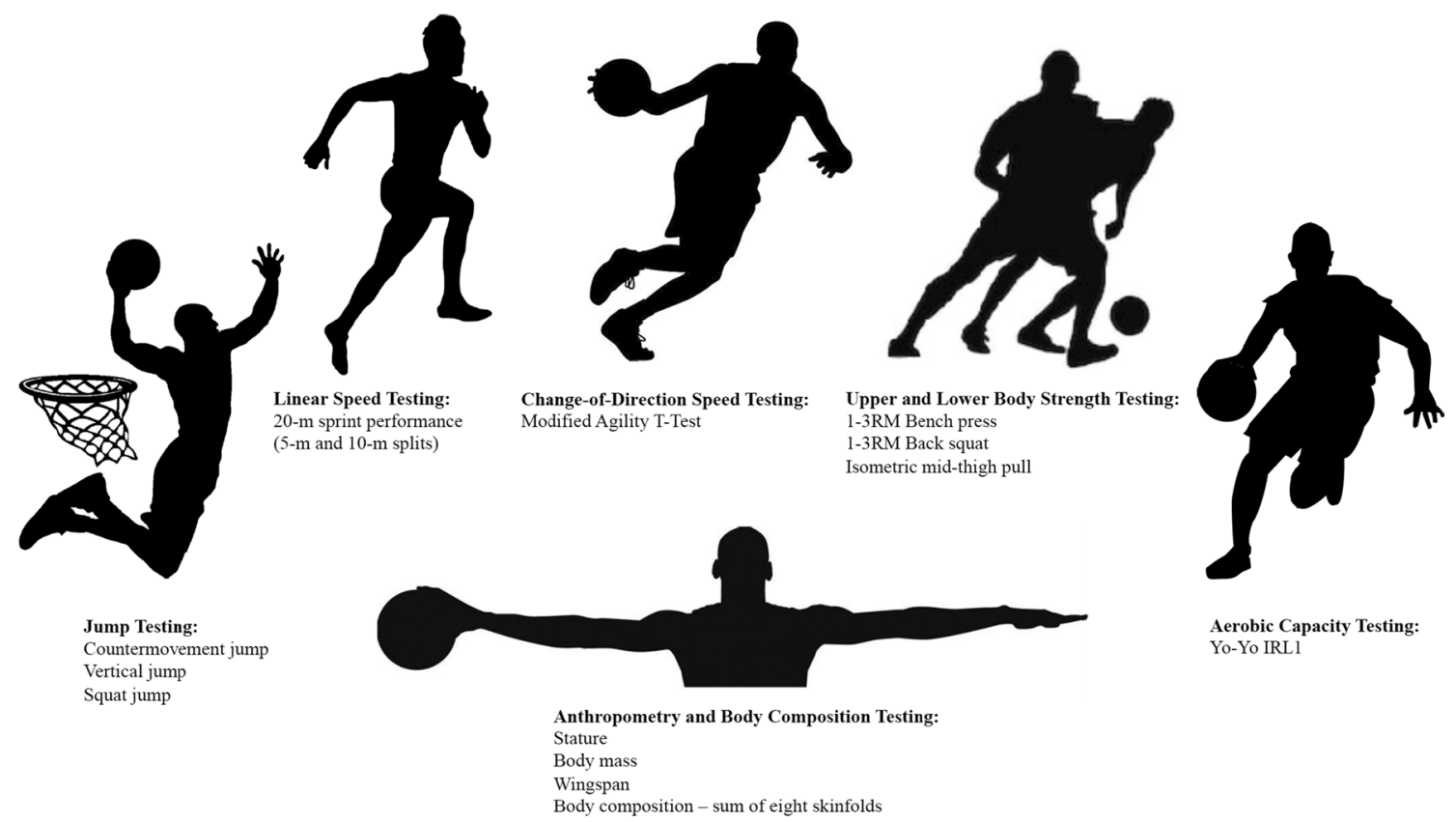

Fig. 5 Recommendations for testing the physical characteristics of adult male basketball players.

these assessments, the sum of eight skinfolds, reported in $\mathrm{mm}$, is suggested because of the low associated cost, relative ease of implementation, and the ability to gather reliable results provided tester competency as outlined by Kasper et al. [147]. With the high prevalence of jumping during basketball match-play [12, 20], a range of jumping tests have been recommended, including the CMJ, VJ, SJ, and the bilateral hopping test, with each jump test assessing different characteristics. For measures of linear sprint speed and change-of-direction speed, 20-m linear sprints and a modified Agility $T$-Test are recommended, respectively. Because of the relatively short length of a basketball court ( $28 \mathrm{~m})$, a 20 -m sprint with 5-m and 10-m split times also recorded may be more appropriate to assess linear sprint performance compared with tests across longer distances that have been adopted in some studies (e.g., $40 \mathrm{~m}[56,93])$. The modified Agility $T$-Test is recommended as the distances covered during the test are limited to $5 \mathrm{~m}$ in any direction, before requiring a change of direction. The shorter distances are more reflective of match demands compared with the traditional Agility $T$-Test [112]. To assess agility, further research is required to develop an agility test that is repeatable, practical to implement, and reflects the demands of basketball. The Y-shaped Agility test may provide basketball practitioners with insight into the agility of players, although the movement demands are not specific to basketball matchplay. Therefore, a definitive agility test for use in adult male basketball players cannot be recommended at this stage.

For measures of strength, 1-3RM during the back squat and bench press are suggested given their ability to provide data that can be monitored over time, inform training prescription, and provide baseline strength measurements for return to play protocols following injury. While the need to produce force is undoubtedly important in the production of power, it may also help support players in maintaining or securing an advantageous position during matches (e.g., backing down an opponent in the low post). It is also recommended that during strength testing, as players are building towards their maximal effort, the velocity of submaximal loads are monitored with an LPT [36]. Use of linear position transducers will support the development of load-velocity profiles in players, which can be used to assess changes in force production with submaximal loads, enhance training prescription, and monitor fatigue [171]. It should also be noted that an IMTP could be a feasible alternative if movement proficiency and competency during common resistance training exercises is lacking. Because of the variability amongst anaerobic tests in the literature, no definitive anaerobic capacity test has been recommended. However, if researchers and practitioners wish to assess anaerobic capacity, an ecologically valid and reliable test should be considered. Finally, the use of the Yo-Yo IRL1 has been suggested to assess aerobic capacity due to its validity, reliability, and feasibility [193, 194]. It is acknowledged that the gold standard gas analysis may provide improved accuracy in measuring $\mathrm{VO}_{2 \max }$; however, because of the constraints commonly associated with this testing (e.g., predominantly in laboratory settings, time, cost, non-basketball-specific movement patterns in test protocols), an efficient on-court solution has been recommended that has been repeatedly 
Table 13 Recommended tests and outcome variables to assess the physical characteristics of adult male basketball players

\begin{tabular}{|c|c|c|c|c|}
\hline Characteristic & Test & Outcome variable & Technical note & Citation count \\
\hline \multirow[t]{3}{*}{ Anthropometry } & Height (cm) & & \multirow{3}{*}{$\begin{array}{l}\text { Measuring anthropometry at the } \\
\text { beginning of the testing battery is } \\
\text { recommended while players are } \\
\text { in a rested state }\end{array}$} & 114 \\
\hline & Body mass (kg) & & & 114 \\
\hline & Wingspan $(\mathrm{cm})$ & & & 3 \\
\hline Body composition & Eight-site skinfolds & Sum of eight skinfolds (mm) & $\begin{array}{l}\text { High inter-tester variability exists. } \\
\text { To gather reliable results, an } \\
\text { experienced tester should conduct } \\
\text { this test. Preferably, the same } \\
\text { practitioner is recommended to } \\
\text { administer this test on different } \\
\text { occasions. An estimation equa- } \\
\text { tion specific to the population is } \\
\text { recommended. Sites used should } \\
\text { be reported and used consistently }\end{array}$ & 2 \\
\hline \multirow[t]{3}{*}{ Muscular power } & Countermovement jump & $\begin{array}{l}\text { Jump height }(\mathrm{cm}) \\
\text { Mean concentric relative power } \\
(\mathrm{W} / \mathrm{kg})\end{array}$ & $\begin{array}{l}\text { To reduce error introduced with } \\
\text { movement strategies, it is recom- } \\
\text { mended that height is calculated } \\
\text { using impulse from a force plat- } \\
\text { form. Relative mean power can } \\
\text { be used as an auxiliary to support } \\
\text { interpretation of jump data }\end{array}$ & 46 \\
\hline & Vertical jump & $\begin{array}{l}\text { Jump height }(\mathrm{cm}) \\
\text { Mean concentric relative power } \\
(\mathrm{W} / \mathrm{kg})\end{array}$ & See above & 33 \\
\hline & Squat jump & $\begin{array}{l}\text { Jump height }(\mathrm{cm}) \\
\text { Mean concentric relative power } \\
(\mathrm{W} / \mathrm{kg})\end{array}$ & $\begin{array}{l}\text { Must carefully monitor force-time } \\
\text { output to ensure no preparatory } \\
\text { countermovement is used. Squat } \\
\text { jump height can be coupled with } \\
\text { countermovement jump height to } \\
\text { support monitoring and prescrip- } \\
\text { tion through calculation of the } \\
\text { 'eccentric utilisation ratio' }\end{array}$ & 19 \\
\hline Linear speed & 20-m sprint & $\begin{array}{l}\text { Time intervals }(\mathrm{s}) \\
\text { Momentum }(\mathrm{kg} \cdot \mathrm{m} / \mathrm{s})\end{array}$ & $\begin{array}{l}\text { Performance time measured at } \\
\text { 5-m, } 10-\mathrm{m} \text { and } 20-\mathrm{m} \text { inter- } \\
\text { vals. Acceleration over each } \\
\text { interval should be calcu- } \\
\text { lated. Starting position must be } \\
\text { standardised (e.g. } 50 \mathrm{~cm} \text { from } \\
\text { first interval) }\end{array}$ & 20 \\
\hline Change-of-direction speed & Modified agility $T$-test & Time (s) & $\begin{array}{l}\text { Total distance covered is } 20 \mathrm{~m} \text {, } \\
\text { with no more than } 5 \mathrm{~m} \text { covered } \\
\text { before requiring a change of } \\
\text { direction. Player must remain } \\
\text { facing forwards for the duration } \\
\text { of the test }\end{array}$ & 1 \\
\hline Strength & 1-3RM Back squat & $\begin{array}{l}\text { Absolute load }(\mathrm{kg}) \\
\text { Relative strength } \\
\text { Load-velocity profile }\end{array}$ & $\begin{array}{l}\text { Players must be competent with } \\
\text { technique to safely implement } \\
\text { test. Squat depth must be stand- } \\
\text { ardised. Additionally, during sub- } \\
\text { maximal efforts, a linear position } \\
\text { transducer should be used to } \\
\text { develop individualised load- } \\
\text { velocity profiles. Dependent upon } \\
\text { athlete technical proficiency, an } \\
\text { isometric mid-thigh pull may be } \\
\text { an alternate or supplementary test }\end{array}$ & 8 \\
\hline
\end{tabular}


Table 13 (continued)

\begin{tabular}{|c|c|c|c|c|}
\hline Characteristic & Test & Outcome variable & Technical note & Citation count \\
\hline & 1-3RM Bench press & $\begin{array}{l}\text { Absolute load }(\mathrm{kg}) \\
\text { Relative strength } \\
\text { Load-velocity profile }\end{array}$ & $\begin{array}{l}\text { Players must be competent with } \\
\text { the technique to safely imple- } \\
\text { ment test. Bench press range of } \\
\text { motion must be standardised. } \\
\text { Load-velocity profiles should } \\
\text { be developed from sub-maximal } \\
\text { loads using a linear position } \\
\text { transducer }\end{array}$ & 14 \\
\hline Aerobic capacity & Yo-Yo IRL1 & $\begin{array}{l}\text { Finishing level } \\
\text { Estimated } \mathrm{VO}_{2 \max }(\mathrm{mL} / \mathrm{kg} / \mathrm{min})\end{array}$ & $\begin{array}{l}\text { Yo-Yo IRL1 must be completed on } \\
\text { the same flooring (e.g. court) and } \\
\text { in similar environmental condi- } \\
\text { tions (e.g. consistent temperature) }\end{array}$ & 12 \\
\hline
\end{tabular}

$R M$ repetition maximum, $V O_{2 \max }$ maximum oxygen uptake, Yo-Yo IRL1 Yo-Yo Intermittent Recovery Test Level 1

used in the literature to assess several samples of adult male basketball players [28, 65, 67, 72, 137].

\section{Conclusions}

This review collates all tests and outcome variables used to assess the physical characteristics of adult male basketball players in the literature to date. The number of tests and outcome variables identified confirm that a gold-standard testing battery for assessing the physical characteristics of basketball players does not exist. While it appears basketball practitioners are prioritising the assessment of specific physical characteristics (i.e., anthropometrics, muscular power, linear speed, change-of-direction speed, agility, strength, anaerobic capacity, and anaerobic capacity), the methods of assessment often vary in regard to technology used (e.g., force platform vs jump mat), variables reported (e.g., mean jump height from multiple attempts $v s$. peak jump height) and test protocols implemented (e.g., number of jumps permitted during a jump test). Further, the varying levels of inherent validity and reliability across the spectrum of tests reported make the establishment of normative data challenging and the comparison of physical characteristics across studies difficult to make in basketball players. To develop meaningful normative data, basketball practitioners must develop standardised testing protocols that are reproducible and reflective of match demands. Developing league-wide and federation-wide testing batteries would allow for the longitudinal assessment of players in large cohorts and the establishment of minimum physical standards for playing positions and competition levels.

Supplementary Information The online version contains supplementary material available at https://doi.org/10.1007/s40279-021-01626-3.

\section{Declarations}

Funding At no point was funding received by any of the authors for the writing of this manuscript.

Conflict of interest Matthew Morrison, David Martin, Scott Talpey, Aaron Scanlan, Jace Delaney, Shona Halson, and Jonathon Weakley declare they have no conflicts of interest relevant to the content of this review.

Ethical approval Not applicable.

Consent to participate Not applicable.

Consent for publication Not applicable.

Availability of data and material All data and materials reported in this systematic review are from peer-reviewed publications and publicly available data from official organisation websites (https://www.nba. com/stats/draft/combine/). All of the extracted data are included in the manuscript and supplementary files.

Code availability Not applicable.

Authors' contributions MM and JW conceptualised the review and criteria. MM and JW completed the screening and data extraction of all data within this manuscript. ST, ATS, and DTM assisted with the formulation of clearly stated objectives, refinement of final methodology, and contributed to the interpretation of findings. MM, JW, ATS, JD, and $\mathrm{SH}$ completed the writing of the manuscript. All authors reviewed and refined the manuscript.

Open Access This article is licensed under a Creative Commons Attribution 4.0 International License, which permits use, sharing, adaptation, distribution and reproduction in any medium or format, as long as you give appropriate credit to the original author(s) and the source, provide a link to the Creative Commons licence, and indicate if changes were made. The images or other third party material in this article are included in the article's Creative Commons licence, unless indicated otherwise in a credit line to the material. If material is not included in the article's Creative Commons licence and your intended use is not permitted by statutory regulation or exceeds the permitted use, you will need to obtain permission directly from the copyright holder. To view a copy of this licence, visit http://creativecommons.org/licenses/by/4.0/. 


\section{References}

1. International Centre for Sports Studies (CIES) CIES Sports Observatory 2019. International Basketball Migration Report 2019. http://www.fiba.basketball/documents/ibmr2019.pdf. Accessed 13 May 2020.

2. International Basketball Federation (FIBA). Rule differences. 2019. https://www.fiba.basketball/rule-differences. Accessed 22 Dec 2021.

3. Lockie RG, Beljic A, Ducheny S, Kammerer J, Dawes JJ. Relationships between playing time and selected NBA combine test performance in Division I Mid-Major Basketball Players. Int J Exerc Sci. 2020;13(4):583-96.

4. Scanlan AT, Tucker PS, Dalbo VJ. A comparison of linear speed, closed-skill agility, and open-skill agility qualities between backcourt and frontcourt adult semiprofessional male basketball players. J Strength Cond Res. 2014;28(5):1319-27.

5. Bredt SG, Torres JO, Diniz LB, Praça GM, Andrade AG, Morales JC, et al. Physical and physiological demands of basketball small-sided games: the influence of defensive and time pressures. Biol Sport. 2020;37(2):131.

6. Ferioli D, Rampinini E, Martin M, Rucco D, La Torre A, Petway $\mathrm{A}$, et al. Influence of ball possession and playing position on the physical demands encountered during professional basketball games. Biol Sport. 2020;37(3):269.

7. Ferioli D, Schelling X, Bosio A, La Torre A, Rucco D, Rampinini E. Match activities in basketball games: comparison between different competitive levels. J Strength Cond Res. 2020;34(1):172-82.

8. Fox JL, Stanton R, Scanlan AT. A comparison of training and competition demands in semiprofessional male basketball players. Res Q Exerc Sport. 2018;89(1):103-11.

9. McLean BD, Strack D, Russell J, Coutts AJ. Quantifying physical demands in the national basketball association: challenges around developing best-practice models for athlete care and performance. Int J Sports Physiol Perform. 2019;14(4):414-20.

10. Petway AJ, Freitas TT, Calleja-González J, Medina Leal D, Alcaraz PE. Training load and match-play demands in basketball based on competition level: a systematic review. PLoS ONE. 2020;15(3):e0229212.

11. Russell JL, McLean BD, Impellizzeri FM, Strack DS, Coutts AJ. Measuring physical demands in basketball: an explorative systematic review of practices. Sports Med. 2020;1-32.

12. Stojanović E, Stojiljković N, Scanlan AT, Dalbo VJ, Berkelmans DM, Milanović Z. The activity demands and physiological responses encountered during basketball match-play: a systematic review. Sports Med. 2018;48(1):111-35.

13. Taylor JB, Wright AA, Dischiavi SL, Townsend MA, Marmon AR. Activity demands during multi-directional team sports: a systematic review. Sports Med. 2017;47(12):2533-51.

14. Puente C, Abian-Vicen J, Areces F, Lopez R, Del Coso J. Physical and physiological demands of experienced male basketball players during a competitive game. J Strength Cond Res. 2017;31(4):956-62.

15. Cormery B, Marcil M, Bouvard M. Rule change incidence on physiological characteristics of elite basketball players: a 10-year-period investigation. Br J Sports Med. 2008;42(1):25-30.

16. Ferioli D, Rampinini E, Bosio A, La Torre A, Azzolini M, Coutts AJ. The physical profile of adult male basketball players: differences between competitive levels and playing positions. J Sports Sci. 2018;36(22):2567-74.

17. Erkmen N, Suveren S, Goktepe AS. Effects of exercise continued until anaerobic threshold on balance performance in male basketball players. J Hum Kinet. 2012;33:73-9.
18. Gocentas A, Jascaniniene N, Poprzecki S, Jaszczanin J, Juozulynas A. Position-related differences in cardiorespiratory functional capacity of elite basketball players. J Hum Kinet. 2011;30:145-52.

19. Gocentas A, Juozulynas A, Obelenis V, Andziulis A, Landor A. Patterns of cardiovascular and ventilatory response to maximal cardiopulmonary test in elite basketball players. Medicina (Kaunas). 2005;41(8):698-704.

20. Scanlan A, Dascombe B, Reaburn P. A comparison of the activity demands of elite and sub-elite Australian men's basketball competition. J Sports Sci. 2011;29(11):1153-60.

21. Scanlan A, Humphries B, Tucker PS, Dalbo V. The influence of physical and cognitive factors on reactive agility performance in men basketball players. J Sports Sci. 2014;32(4):367-74.

22. Weakley J, Chalkley D, Johnston R, García-Ramos A, Townshend A, Dorrell H, et al. Criterion validity, and interunit and between-day reliability of the FLEX for measuring barbell velocity during commonly used resistance training exercises. J Strength Cond Res. 2020.

23. Bradic A, Bradic J, Pasalic E, Markovic G. Isokinetic leg strength profile of elite male basketball players. J Strength Cond Res. 2009;23(4):1332-7.

24. Buśko K. Training-induced changes in the topography of muscle torques and maximal muscle torques in basketball players. Biol Sport. 2012;29(1):77-83.

25. Hadzic V, Erculj F, Bracic M, Dervisevic E. Bilateral concentric and eccentric isokinetic strength evaluation of quadriceps and hamstrings in basketball players. Coll Antropol. 2013;37(3):859-65.

26. Mangine GT, Hoffman JR, Gonzalez AM, Jajtner AR, Scanlon $\mathrm{T}$, Rogowski JP, et al. Bilateral differences in muscle architecture and increased rate of injury in national basketball association players. J Athl Train. 2014;49(6):794-9.

27. Boone J, Bourgois J. Morphological and physiological profile of elite basketball players in Belgian. Int J Sports Physiol Perform. 2013;8(6):630-8.

28. Montgomery PG, Pyne DB, Hopkins WG, Dorman JC, Cook $\mathrm{K}$, Minahan CL. The effect of recovery strategies on physical performance and cumulative fatigue in competitive basketball. J Sports Sci. 2008;26(11):1135-45.

29. Ramirez-Campillo R, Gentil P, Moran J, Dalbo VJ, Scanlan AT. Dribble deficit enables measurement of dribbling speed independent of sprinting speed in collegiate, male, basketball players. J Strength Cond Res. 2019.

30. Scanlan AT, Wen N, Spiteri T, Milanovic Z, Conte D, Guy $\mathrm{JH}$, et al. Dribble deficit: a novel method to measure dribbling speed independent of sprinting speed in basketball players. J Sports Sci. 2018;36(22):2596-602.

31. Ahmed TAE. Improving musculoskeletal fitness and the performance enhancement of basketball skills through neuromuscular training program. J Hum Sport Exerc. 2015;10(3).

32. Ziv G, Lidor R. Physical attributes, physiological characteristics, on-court performances and nutritional strategies of female and male basketball players. Sports Med. 2009;39(7):547-68.

33. Mancha-Triguero D, Garcia-Rubio J, González-Calleja J, Ibanez SJ. Physical fitness in basketball players: a systematic review. J Sports Med Phys Fit. 2019.

34. Liberati A, Altman DG, Tetzlaff J, Mulrow C, Gøtzsche PC, Ioannidis JP, et al. The PRISMA statement for reporting systematic reviews and meta-analyses of studies that evaluate health care interventions: explanation and elaboration. J Clin Epidemiol. 2009;62(10):e1-34.

35. Owen C, Till K, Weakley J, Jones B. Testing methods and physical qualities of male age grade rugby union players: a systematic review. PLoS ONE. 2020;15(6):e0233796. 
36. Weakley J, Morrison M, García-Ramos A, Johnston R, James $\mathrm{L}$, Cole $\mathrm{MH}$. The validity and reliability of commercially available resistance training monitoring devices: a systematic review. Sports Med. 2021.

37. Downs SH, Black N. The feasibility of creating a checklist for the assessment of the methodological quality both of randomised and non-randomised studies of health care interventions. J Epidemiol Community Health. 1998;52(6):377-84.

38. Latin RW, Berg K, Baechle T. Physical and performance characteristics of NCAA division I male basketball players. J Strength Cond Res. 1994;8(4):214-8.

39. Pliauga V, Lukonaitiene I, Kamandulis S, Skurvydas A, Sakalauskas R, Scanlan AT, et al. The effect of block and traditional periodization training models on jump and sprint performance in collegiate basketball players. Biol Sport. 2018;35(4):373-82.

40. Rohatgi A. WebPlotDigitizer V 4.4. 2020.

41. Drevon D, Fursa SR, Malcolm AL. Intercoder reliability and validity of WebPlotDigitizer in extracting graphed data. Behav Modif. 2017;41(2):323-39.

42. WarnerMedia LLC's Turner Sports \& Entertainment Digital Network. NBA Media Ventures LLC. NBA.com. NBA advanced stats. 2021. https://www.nba.com/stats/draft/combine/. Accessed 22 Feb 2021.

43. Angyan L, Teczely T, Zalay Z, Karsai I. Relationship of anthropometrical, physiological and motor attributes to sport-specific skills. Acta Physiol Hung. 2003;90(3):225-31.

44. Petway AJ, Freitas TT, Calleja-González J, Torres-Ronda L, Alcaraz PE. Seasonal variations in game activity profiles and players' neuromuscular performance in Collegiate Division I Basketball: non-conference vs. conference tournament. Front Sports Act Living. 2020.

45. Milanović L, Jukić I, Dadić M, Vučetić V, Šentija D. Is there any difference in fitness profiles among the Croatian basketball players? Position specific analysis. Kinesiology. 2019;51(2):276-84.

46. Young WB, Dawson B, Henry GJ. Agility and change-ofdirection speed are independent skills: implications for training for agility in invasion sports. Int J Sports Sci Coach. 2015;10(1):159-69.

47. Sheppard JM, Young WB. Agility literature review: classifications, training and testing. J Sports Sci. 2006;24(9):919-32.

48. Pehar M, Sekulic D, Sisic N, Spasic M, Uljevic O, Krolo A, et al. Evaluation of different jumping tests in defining position-specific and performance-level differences in high level basketball players. Biol Sport. 2017;34(3):263.

49. Sekulic D, Pehar M, Krolo A, Spasic M, Uljevic O, CallejaGonzalez J, et al. Evaluation of basketball-specific agility: applicability of preplanned and nonplanned agility performances for differentiating playing positions and playing levels. J Strength Cond Res. 2017;31(8):2278-88.

50. Fields JB, Merrigan JJ, White JB, Jones MT. Seasonal and longitudinal changes in body composition by sport-position in NCAA Division I basketball athletes. Sports. 2018;6(3):85.

51. Dawes JJ, Spiteri T. Relationship between pre-season testing performance and playing time among NCAA DII basketball players. Sport Exerc Med Open J. 2016;2(2).

52. Gryko K, Kopiczko A, Mikolajec K, Stasny P, Musalek M. Anthropometric variables and somatotype of young and professional male basketball players. Sports (Basel). 2018;6(1).

53. Caterisano A, Patrick BT, Edenfield WL, Batson MJ. The effects of a basketball season on aerobic and strength parameters among college men: starters vs reserves. J Strength Cond Res. 1997;11(1):21-4.

54. Ferioli D, Bosio A, Bilsborough JC, La Torre A, Tornaghi M, Rampinini E. The preparation period in basketball: training load and neuromuscular adaptations. Int J Sports Physiol Perform. 2018;13(8):991-9.

55. Ferioli D, Bosio A, Zois J, La Torre A, Rampinini E. Seasonal changes in physical capacities of basketball players according to competitive levels and individual responses. PLoS ONE. 2020;15(3):e0230558.

56. Kariyawasam A, Ariyasinghe A, Rajaratnam A, Subasinghe P. Comparative study on skill and health related physical fitness characteristics between national basketball and football players in Sri Lanka. BMC Res Notes. 2019;12(1):397.

57. Narazaki K, Berg K, Stergiou N, Chen B. Physiological demands of competitive basketball. Scand J Med Sci Sports. 2009; 19(3):425-32.

58. Maggioni MA, Bonato M, Stahn A, Torre AL, Agnello L, Vernillo G, et al. Effects of ball drills and repeated-sprint-ability training in basketball players. Int J Sports Physiol Perform. 2019;14(6):757-64.

59. de Sousa FL, Paes PP, Mortatti AL, Perez AJ, Cyrino ES, de Lima-Júnior DRAA, et al. Effect of different warm-up strategies on countermovement jump and sprint performance in basketball players. Isokinet Exerc Sci. 2018;26(3):219-25.

60. Scanlan AT, Tucker PS, Dalbo VJ. The importance of open- and closed-skill agility for team selection of adult male basketball players. J Sports Med Phys Fit. 2015;55(5):390-6.

61. Alemdaroğlu $U$. The relationship between muscle strength, anaerobic performance, agility, sprint ability and vertical jump performance in professional basketball players. J Hum Kinet. 2012;31:149-58.

62. Annino G, Ruscello B, Lebone P, Palazzo F, Lombardo M, Padua E, et al. Acute effects of static and dynamic stretching on jump performance after $15 \mathrm{~min}$ of reconditioning shooting phase in basketball players. J Sports Med Phys Fitness. 2017;57(4):330-7.

63. Aoki MS, Ronda LT, Marcelino PR, Drago G, Carling C, Bradley $\mathrm{PS}$, et al. Monitoring training loads in professional basketball players engaged in a periodized training program. J Strength Cond Res. 2017;31(2):348-58.

64. Barrera-Domínguez FJ, Almagro BJ, Tornero-Quiñones I, SáezPadilla J, Sierra-Robles Á, Molina-López J. Decisive factors for a greater performance in the change of direction and its angulation in male basketball players. Int J Environ Res Public Health. 2020;17(18):6598.

65. Ben Abdelkrim N, Chaouachi A, Chamari K, Chtara M, Castagna C. Positional role and competitive-level differences in elite-level men's basketball players. J Strength Cond Res. 2010;24(5):1346-55.

66. Buśko K, Pastuszak A, Lipinska M, Lipinska M, Gryko K. Somatotype variables related to strength and power output in male basketball players. Acta Bioeng Biomech. 2017;19(2):161-7.

67. Chaouachi A, Brughelli M, Chamari K, Levin GT, Ben Abdelkrim N, Laurencelle L, et al. Lower limb maximal dynamic strength and agility determinants in elite basketball players. J Strength Cond Res. 2009;23(5):1570-7.

68. Chen WH, Wu HJ, Lo SL, Chen H, Yang WW, Huang CF, et al. Eight-week battle rope training improves multiple physical fitness dimensions and shooting accuracy in collegiate basketball players. J Strength Cond Res. 2018;32(10):2715-24.

69. Dello Iacono A, Beato M, Halperin I. The effects of cluster-set and traditional-set postactivation potentiation protocols on vertical jump performance. Int J Sports Physiol Perform. 2020;15(4).

70. Freitas TT, Calleja-Gonzalez J, Alarcon F, Alcaraz PE. Acute effects of two different resistance circuit training protocols on performance and perceived exertion in semiprofessional basketball players. J Strength Cond Res. 2016;30(2):407-14.

71. Freitas TT, Calleja-Gonzalez J, Carlos-Vivas J, Marin-Cascales E, Alcaraz PE. Short-term optimal load training vs a modified 
complex training in semi-professional basketball players. J Sports Sci. 2019;37(4):434-42.

72. Gomes JH, Rebello Mendes R, Almeida MBd, Zanetti MC, Leite GdS, Figueira Júnior AJ. Relationship between physical fitness and game-related statistics in elite professional basketball players: regular season vs. playoffs. Motriz Rev Ed Fís. 2017;23(2).

73. Heishman AD, Curtis MA, Saliba E, Hornett RJ, Malin SK, Weltman AL. Noninvasive assessment of internal and external player load: implications for optimizing athletic performance. $\mathrm{J}$ Strength Cond Res. 2018;32(5):1280-7.

74. Heishman AD, Curtis MA, Saliba EN, Hornett RJ, Malin SK, Weltman AL. Comparing performance during morning vs. afternoon training sessions in intercollegiate basketball players. J Strength Cond Res. 2017;31(6):1557-62.

75. Heishman AD, Daub BD, Miller RM, Freitas ED, Bemben MG. Monitoring external training loads and neuromuscular performance for division I basketball players over the preseason. $\mathrm{J}$ Sports Sci Med. 2020;19(1):204.

76. Heishman AD, Daub BD, Miller RM, Freitas ED, Frantz BA, Bemben MG. Countermovement jump reliability performed with and without an arm swing in NCAA division 1 intercollegiate basketball players. J Strength Cond Res. 2020;34(2):546-58.

77. Jallai T, Ereline J, Kums T, Aibast H, Gapeyeva H, Pääsuke M. Postural control and vertical jumping performance in adolescent and adult male basketball players. Acta Kinesiol Univ Tartu. 2012;17.

78. Khlifa R, Aouadi R, Hermassi S, Chelly MS, Jlid MC, Hbacha H, et al. Effects of a plyometric training program with and without added load on jumping ability in basketball players. J Strength Cond Res. 2010;24(11):2955-61.

79. Köklü Y, Alemdaroglu U, Kocak FU, Erol AE, Findikoglu G. Comparison of chosen physical fitness characteristics of Turkish professional basketball players by division and playing position. J Hum Kinet. 2011;30:99-106.

80. Laplaud D, Hug F, Menier R. Training-induced changes in aerobic aptitudes of professional basketball players. Int J Sports Med. 2004;25(2):103-8.

81. Maffiuletti NA, Cometti G, Amiridis IG, Martin A, Pousson M, Chatard JC. The effects of electromyostimulation training and basketball practice on muscle strength and jumping ability. Int $\mathbf{J}$ Sports Med. 2000;21(6):437-43.

82. Mandic R, Jakovljevic S, Jaric S. Effects of countermovement depth on kinematic and kinetic patterns of maximum vertical jumps. J Electromyogr Kinesiol. 2015;25(2):265-72.

83. Miura K, Yamamoto M, Tamaki H, Zushi K. Determinants of the abilities to jump higher and shorten the contact time in a running 1-legged vertical jump in basketball. J Strength Cond Res. 2010;24(1):201-6.

84. Ostojic SM, Mazic S, Dikic N. Profiling in basketball: physical and physiological characteristics of elite players. J Strength Cond Res. 2006;20(4):740-4.

85. Pehar M, Sisic N, Sekulic D, Coh M, Uljevic O, Spasic M, et al. Analyzing the relationship between anthropometric and motor indices with basketball specific pre-planned and non-planned agility performances. J Sports Med Phys Fit. 2018;58(7-8):1037-44.

86. Pliauga V, Kamandulis S, Dargeviciute G, Jaszczanin J, Kliziene I, Stanislovaitiene J, et al. The effect of a simulated basketball game on players' sprint and jump performance, temperature and muscle damage. J Hum Kinet. 2015;27(46):167-75.

87. Pojskić H, Šeparović V, Muratović M, Užičanin E. The relationship between physical fitness and shooting accuracy of professional basketball players. Motriz Rev Ed Fis. 2014;20(4):408-17.

88. Pojskic H, Separovic V, Uzicanin E, Muratovic M, Mackovic S. Positional role differences in the aerobic and anaerobic power of elite basketball players. J Hum Kinet. 2015;22(49):219-27.

89. Pojskic H, Sisic N, Separovic V, Sekulic D. Association between conditioning capacities and shooting performance in professional basketball players: an analysis of stationary and dynamic shooting skills. J Strength Cond Res. 2018;32(7):1981-92.

90. Ponce-Gonzalez JG, Olmedillas H, Calleja-Gonzalez J, Guerra B, Sanchis-Moysi J. Physical fitness, adiposity and testosterone concentrations are associated to playing position in professional basketballers. Nutr Hosp. 2015;31(6):2624-32.

91. Rodriguez-Rosell D, Mora-Custodio R, Franco-Marquez F, Yanez-Garcia JM, Gonzalez-Badillo JJ. Traditional vs. sportspecific vertical jump tests: reliability, validity, and relationship with the legs strength and sprint performance in adult and teen soccer and basketball players. J Strength Cond Res. 2017;31(1):196-206.

92. Schiltz M, Lehance C, Maquet D, Bury T, Crielaard JM, Croisier JL. Explosive strength imbalances in professional basketball players. J Athl Train. 2009;44(1):39-47.

93. Shalfawi SA, Sabbah A, Kailani G, Tonnessen E, Enoksen E. The relationship between running speed and measures of vertical jump in professional basketball players: a field-test approach. J Strength Cond Res. 2011;25(11):3088-92.

94. Stojanovic MD, Ostojic SM, Calleja-Gonzalez J, Milosevic Z, Mikic M. Correlation between explosive strength, aerobic power and repeated sprint ability in elite basketball players. J Sports Med Phys Fit. 2012;52(4):375-81.

95. Xie T, Crump KB, Ni R, Meyer CH, Hart JM, Blemker SS, et al. Quantitative relationships between individual lower-limb muscle volumes and jump and sprint performances of basketball players. J Strength Cond Res. 2020;34(3):623-31.

96. Ciacci S, Bartolomei S. The effects of two different explosive strength training programs on vertical jump performance in basketball. J Sports Med Phys Fit. 2018;58(10):1375-82.

97. Asadi A, Saez de Villarreal E, Arazi H. The effects of plyometric type neuromuscular training on postural control performance of male team basketball players. J Strength Cond Res. 2015;29(7):1870-5

98. Balabinis CP, Psarakis CH, Moukas M, Vassiliou MP, Behrakis PK. Early phase changes by concurrent endurance and strength training. J Strength Cond Res. 2003;17(2):393-401.

99. Balsalobre-Fernández C, Tejero-González CM, del CampoVecino J, Bachero-Mena B, Sánchez-Martínez J. Relationships among repeated sprint ability, vertical jump performance and upper-body strength in professional basketball players. Arch Med Deporte. 2014;31(161):148-53.

100. Balsalobre-Fernández C, Tejero-González CM, del CampoVecino J, Bachero-Mena B, Sánchez-Martínez J. Differences of muscular performance between professional and young basketball players. (Diferencias en el rendimiento muscular entre jugadores de baloncesto profesionales y jóvenes). Cult Cienc Deporte. 2016;11(31):61-5.

101. Heishman A, Brown B, Daub B, Miller R, Freitas E, Bemben $M$. The influence of countermovement jump protocol on reactive strength index modified and flight time: contraction time in collegiate basketball players. Sports. 2019;7(2):37.

102. Hoffman J, Maresh C, Armstrong L, Kraemer W. Effects of offseason and in-season resistance training programs on a collegiate male basketball team. J Hum Muscle Perform. 1991;1(2):48-55. 
103. Hoffman JR, Fry AC, Howard R, Maresh CM, Kraemer WJ. Strength, speed and endurance changes during the course of a division I basketball season. J Strength Cond Res. 1991;5(3):144-9.

104. Hoffman JR, Tenenbaum G, Maresh CM, Kraemer WJ. Relationship between athletic performance tests and playing time in elite college basketball players. J Strength Cond Res. 1996;10(2):67-71.

105. Hunter GR, Hilyer J, Forster MA. Changes in fitness during 4 years of intercollegiate basketball. J Strength Cond Res. 1993;7(1):26-9.

106. Kipp K, Kiely M, Giordanelli M, Malloy P, Geiser C. Jointand subject-specific strategies in male basketball players across a range of countermovement jump heights. J Sports Sci. 2020;38(6):652-7.

107. Korkmaz C, Karahan M. A comparative study on the physical fitness and performance of male basketball players in different divisions. J Phys Educ Sport Sci. 2012;6(1).

108. Lehnert M, Hůlka K, Malý T, Fohler J, Zahálka F. The effects of a 6 week plyometric training programme on explosive strength and agility in professional basketball players. Acta Gymnica. 2013;43(4):7-15.

109. Nikolaidis P, Calleja-González J, Padulo J. The effect of age on positional differences in anthropometry, body composition, physique and anaerobic power of elite basketball players. Sport Sci Health. 2014;10(3):225-33.

110. Rauch J, Leidersdorf E, Reeves T, Borkan L, Elliott M, Ugrinowitsch C. Different movement strategies in the countermovement jump amongst a large cohort of NBA players. Int J Environ Res Public Health. 2020;17(17):6394.

111. Townsend JR, Bender D, Vantrease WC, Hudy J, Huet K, Williamson $\mathrm{C}$, et al. Isometric midthigh pull performance is associated with athletic performance and sprinting kinetics in Division I Men and Women's Basketball players. J Strength Cond Res. 2019;33(10):2665-73.

112. Poole J, Fox J, Scanlan A. The contribution of linear sprinting and lateral shuffling to change of direction T-test performance in semi-professional, male basketball players. J Aust Strength Cond. 2017;25:6-12.

113. Lockie RG, Jeffriess MD, McGann TS, Callaghan SJ, Schultz AB. Planned and reactive agility performance in semiprofessional and amateur basketball players. Int J Sports Physiol Perform. 2014;9(5):766-71.

114. Delextrat A, Cohen D. Physiological testing of basketball players: toward a standard evaluation of anaerobic fitness. J Strength Cond Res. 2008;22(4):1066-72.

115. Mitić M, Paunović M, Živković M, Stojanović N, Bojić I, Kocić M. Differences in agility and explosive power of basketball players in relation to their positions on the team. FU Phys Ed Sport. 2019;16(2).

116. Myles JR, Lee CM, Kern M. The influence of various recovery modalities on performance tasks in basketball players. Int $\mathbf{J}$ Appl Exerc Physiol. 2017;6(1).

117. Soslu R, Özkan A, Göktepe M. The relationship between anaerobic performances, muscle strength, hamstring/quadriceps ratio, agility, sprint ability and vertical jump in professional basketball players. J Phys Educ Sport Sci. 2016;10(2).

118. Jeffriess MD, Schultz AB, McGann TS, Callaghan SJ, Lockie RG. Effects of preventative ankle taping on planned changeof-direction and reactive agility performance and ankle muscle activity in basketballers. J Sports Sci Med. 2015;14(4):864-76.

119. Gillam GM. Basketball: Identification of anthropometric and physiological characteristics relative to participation in college basketball. Strength Cond J. 1985;7(3):34-6.
120. Hunter GR, Hilyer J. Bridging the gap-research: evaluation of the University of Alabama at Birmingham Men's Basketball Team. Strength Cond J. 1989;11(6):14-5.

121. Parr RB, Hoover R, Wilmore JH, Bachman D, Kerlan RK. Professional basketball players: athletic profiles. Phys Sports Med. 1978;6(4):77-87.

122. Omorczyk J, Ambrozy T, Puszczalowska-Lizis E, Nowak M, Markowski A. Effects of 6-week basketball training using the modified circuit weight method. Balt J Health Phys Act. 2017;9(4):44-54.

123. Gonzalez AM, Hoffman JR, Rogowski JP, Burgos W, Manalo E, Weise K, et al. Performance changes in NBA basketball players vary in starters vs. nonstarters over a competitive season. J Strength Cond Res. 2013;27(3):611-5.

124. Čabarkapa D, Fry AC, Lane MT, Hudy A, Dietz PR, Cain GJ, et al. The importance of lower body strength and power for future success in professional men's basketball. Sport nauke zdr. 2020;10(1).

125. Fatouros IG, Laparidis K, Kambas A, Chatzinikolaou A, Techlikidou E, Katrabasas I, et al. Validity and reliability of the single-trial line drill test of anaerobic power in basketball players. J Sports Med Phys Fit. 2011;51(1):33-41.

126. Sallet P, Perrier D, Ferret JM, Vitelli V, Baverel G. Physiological differences in professional basketball players as a function of playing position and level of play. J Sports Med Phys Fit. 2005;45(3):291-4.

127. Popadic Gacesa JZ, Barak OF, Grujic NG. Maximal anaerobic power test in athletes of different sport disciplines. J Strength Cond Res. 2009;23(3):751-5.

128. Harbili S. Relationship between lower extremity isokinetic strength and anaerobic power in weightlifters, basketball and soccer players. Isokinet Exerc Sci. 2015;23(2):93-100.

129. de Araujo GG, de Barros M-G, Papoti M, Camargo BH, Gobatto CA. Anaerobic and aerobic performances in elite basketball players. J Hum Kinet. 2014;29(42):137-47.

130. Bolonchuk WW, Lukaski HC, Siders WA. The structural, functional, and nutritional adaptation of college basketball players over a season. J Sports Med Phys Fit. 1991;31(2):165-72.

131. Boone J, Deprez D, Bourgois J. Running economy in elite soccer and basketball players: differences among positions on the field. Int J Perform Anal. 2017;14(3):775-87.

132. Chatzinikolaou A, Draganidis D, Avloniti A, Karipidis A, Jamurtas AZ, Skevaki CL, et al. The microcycle of inflammation and performance changes after a basketball match. $\mathbf{J}$ Sports Sci. 2014;32(9):870-82.

133. Dragonea P, Zacharakis E, Kounalakis S, Kostopoulos N, Bolatoglou T, Apostolidis N. Determination of the exercise intensity corresponding with maximal lactate steady state in highlevel basketball players. Res Sports Med. 2019;27(1):112-20.

134. McInnes S, Carlson J, Jones C, McKenna M. The physiological load imposed on basketball players during competition. J Sports Sci. 1995;13(5):387-97.

135. Metaxas TI, Koutlianos N, Sendelides T, Mandroukas A. Preseason physiological profile of soccer and basketball players in different divisions. J Strength Cond Res. 2009;23(6):1704-13.

136. Ferioli D, Bosio A, La Torre A, Carlomagno D, Connolly DR, Rampinini E. Different training loads partially influence physiological responses to the preparation period in Basketball. $\mathrm{J}$ Strength Cond Res. 2018;32(3):790-7.

137. Scanlan AT, Dascombe BJ, Reaburn PR. The construct and longitudinal validity of the basketball exercise simulation test. J Strength Cond Res. 2012;26(2):523-30.

138. Scanlan AT, Dascombe BJ, Reaburn PRJ. Development of the Basketball Exercise Simulation Test: A match-specific basketball fitness test. J Hum Sport Exerc. 2014;9(3):700-12. 
139. Young W, Wilson G, Byrne C. Relationship between strength qualties and performance in standing and run-up vertical jumps. J Sports Med Phys Fitness. 1999;39(4):285.

140. McMahon JJ, Jones PA, Comfort P. Comment on: "Anthropometric and physical qualities of elite male youth rugby league players." Sports Med. 2017;47(12):2667-8.

141. Bobbert MF, Gerritsen KG, Litjens MC, Van Soest AJ. Why is countermovement jump height greater than squat jump height? Med Sci Sports Exerc. 1996;28:1402-12.

142. Gerodimos V, Zafeiridis A, Perkos S, Dipla K, Manou V, Kellis $\mathrm{S}$. The contribution of stretch-shortening cycle and arm-swing to vertical jumping performance in children, adolescents, and adult basketball players. Pediatr Exerc Sci. 2008;20(4):379-89.

143. Crewther BT, McGuigan MR, Gill ND. The ratio and allometric scaling of speed, power, and strength in elite male rugby union players. J Strength Cond Res. 2011;25(7):1968-75.

144. Teramoto M, Cross CL, Rieger RH, Maak TG, Willick SE. Predictive validity of National Basketball Association Draft combine on future performance. J Strength Cond Res. 2018;32(2):396-408.

145. Tomkinson GR, Popovic N, Martin M. Bilateral symmetry and the competitive standard attained in elite and sub-elite sport. J Sports Sci. 2003;21(3):201-11.

146. The International Society for the Advancement of Kinanthropometry (ISAK). https://www.isak.global/Home/Index. Accessed 21 Sept 2021.

147. Kasper AM, Langan-Evans C, Hudson JF, Brownlee TE, Harper LD, Naughton RJ, et al. Come back skinfolds, all is forgiven: a narrative review of the efficacy of common body composition methods in applied sports practice. Nutrients. 2021;13(4):1075.

148. Wen N, Dalbo VJ, Burgos B, Pyne DB, Scanlan AT. Power testing in basketball: current practice and future recommendations. J Strength Cond Res. 2018;32(9):2677-91.

149. Abdelkrim NB, El Fazaa S, El Ati J. Time-motion analysis and physiological data of elite under-19-year-old basketball players during competition. Br J Sports Med. 2007;41(2):69-75.

150. Ziv G, Lidor R. Vertical jump in female and male basketball players: a review of observational and experimental studies. J Sci Med Sport. 2010;13(3):332-9.

151. Cormack SJ, Newton RU, McGuigan MR, Doyle TL. Reliability of measures obtained during single and repeated countermovement jumps. Int J Sports Physiol Perform. 2008;3(2):131-44.

152. McMahon JJ, Lake JP, Comfort P. Reliability of and relationship between flight time to contraction time ratio and reactive strength index modified. Sports. 2018;6(3):81.

153. Halson SL. Monitoring training load to understand fatigue in athletes. Sports Med. 2014;44(2):139-47.

154. Markwick WJ, Bird SP, Tufano JJ, Seitz LB, Haff GG. The intraday reliability of the Reactive Strength Index calculated from a drop jump in professional men's basketball. Int J Sports Physiol Perform. 2015;10(4):482-8.

155. Castagna C, Chaouachi A, Rampinini E, Chamari K, Impellizzeri F. Aerobic and explosive power performance of elite italian regional-level basketball players. J Strength Cond Res. 2009;23(7):1982-7.

156. Leduc C, Tee J, Lacome M, Weakley J, Cheradame J, Ramirez $\mathrm{C}$, et al. Convergent validity, reliability, and sensitivity of a running test to monitor neuromuscular fatigue. Int J Sports Physiol Perform. 2020;1(aop):1-7.

157. Oliver JL, Lloyd RS, Whitney A. Monitoring of in-season neuromuscular and perceptual fatigue in youth rugby players. Eur $\mathbf{J}$ Sport Sci. 2015;15(6):514-22.

158. Fujii K, Yamada Y, Oda S. Skilled basketball players rotate their shoulders more during running while dribbling. Percept Mot Skills. 2010;110(3 Pt 1):983-94.
159. Haugen TA, Breitschadel F, Seiler S. Sprint mechanical properties in handball and basketball players. Int J Sports Physiol Perform. 2019;2:1-7.

160. Mikolajec K, Waskiewicz Z, Maszczyk A, Bacik B, Kurek P, Zajac A. Effects of stretching and strength exercises on speed and power abilities in male basketball players. Isokinet Exerc Sci. 2012;20(1):61-9.

161. Ferioli D, Rampinini E, Bosio A, La Torre A, Maffiuletti NA. Peripheral muscle function during repeated changes of direction in basketball. Int J Sports Physiol Perform. 2019;14(6):739-46.

162. Van Gelder LH, Bartz SD. The effect of acute stretching on agility performance. J Strength Cond Res. 2011;25(11):3014-21.

163. Young W, Farrow D. The importance of a sport-specific stimulus for training agility. Strength Cond J. 2013;35(2):39-43.

164. Henry G, Dawson B, Lay B, Young W. Validity of a reactive agility test for Australian football. Int J Sports Physiol Perform. 2011;6(4):534-45.

165. Sheppard J, Young WB, Doyle T, Sheppard T, Newton RU. An evaluation of a new test of reactive agility and its relationship to sprint speed and change of direction speed. J Sci Med Sport. 2006;9(4):342-9.

166. Gabbett TJ, Kelly JN, Sheppard JM. Speed, change of direction speed, and reactive agility of rugby league players. J Strength Cond Res. 2008;22(1):174-81.

167. Serpell BG, Ford M, Young WB. The development of a new test of agility for rugby league. J Strength Cond Res. 2010;24(12):3270-7.

168. Ilie M, Cristian G. Monitoring the explosive strength parameters in BCM U Pitesti Basketball team. J Phys Educ Sport. 2017;2017(17):2297-300.

169. Ivanovic J, Dopsaj M. Reliability of force-time curve characteristics during maximal isometric leg press in differently trained high-level athletes. Measurement. 2013;46(7):2146-54.

170. Theoharopoulos A, Tsitskaris G, Nikopoulou M, Tsaklis P. Knee strength of professional basketball players./Force du genou de basketteurs professionels. J Strength Cond Res. 2000;14(4):457-63

171. Weakley J, Mann B, Banyard H, McLaren S, Scott T, GarciaRamos A. Velocity-based training: from theory to application. Strength Cond J. 2020:1-19.

172. Pearson M, García-Ramos A, Morrison M, Ramirez-Lopez C, Dalton-Barron N, Weakley J. Velocity loss thresholds reliably control kinetic and kinematic outputs during free weight resistance training. Int J Environ Res Public Health. 2020;17(18):6509.

173. Banyard HG, Tufano JJ, Weakley JJ, Wu S, Jukic I, Nosaka K. Superior changes in jump, sprint, and change-of-direction performance but not maximal strength following 6 weeks of velocitybased training compared with 1-repetition-maximum percentagebased training. Int J Sports Physiol Perform. 2020;1(aop):1-11.

174. Weakley J, McLaren S, Ramirez-Lopez C, García-Ramos A, Dalton-Barron N, Banyard H, et al. Application of velocity loss thresholds during free-weight resistance training: responses and reproducibility of perceptual, metabolic, and neuromuscular outcomes. J Sports Sci. 2020;38(5):477-85.

175. García-Ramos A, Janicijevic D, González-Hernández JM, Keogh JW, Weakley J. Reliability of the velocity achieved during the last repetition of sets to failure and its association with the velocity of the 1-repetition maximum. PeerJ. 2020;8:e8760.

176. Stone MH, O’Bryant HS, Hornsby G, Cunanan A, Mizuguchi $\mathrm{S}$, Suarez DG, et al. Using the isometric mid-thigh pull in the monitoring of weighlifters: $25+$ years of experience. Proform Strength Cond. 2019;54:19-26.

177. Delextrat A, Baliqi F, Clarke N. Repeated sprint ability and stride kinematics are altered following an official match in national-level basketball players. J Sports Med Phys Fit. 2013;53(2):112-8. 
178. Papanikolaou K, Chatzinikolaou A, Pontidis T, Avloniti A, Deli CK, Leontsini D, et al. The Yo-Yo Intermittent Endurance Level 2 Test: reliability of performance scores, physiological responses and overload characteristics in eompetitive soccer, basketball and volleyball players. J Hum Kinet. 2019;67:223-33.

179. Vaquera A, Villa JG, Morante JC, Thomas G, Renfree AJ, Peters DM. Validity and test-retest reliability of the TIVRE-Basket Test for the determination of aerobic power in elite male basketball players. J Strength Cond Res. 2016;30(2):584-7.

180. Lysenko $\mathrm{O}$. The features of functional capabilities of elite basketball players related to game function. Balt J Health Phys Act. 2009;1(1).

181. Tavino LP, Bowers CJ, Archer CB. Effects of basketball on aerobic capacity, anaerobic capacity, and body composition of male college players. J Strength Cond Res. 1995;9(2):75-7.

182. Sanders GJ, Turner Z, Boos B, Peacock CA, Peveler W, Lipping A. Aerobic capacity is related to repeated sprint ability with sprint distances less than 40 meters. Int J Exerc Sci. 2017;10(2): 197 .

183. Leger LA, Lambert J. A maximal multistage 20-m shuttle run test to predict $V \mathrm{O}_{2 \max }$. Eur J Appl Physiol Occup Physiol. 1982;49(1):1-12.

184. Alejandro V, Santiago S, Gerardo VJ, Carlos MJ, Vicente GT. Anthropometric characteristics of Spanish professional basketball players. J Hum Kinet. 2015;27(46):99-106.

185. Gaurav V, Singh M, Singh S. Anthropometric characteristics, somatotyping and body composition of volleyball and basketball players. J Phys Educ. 2010;1(3):28-32.

186. Gerodimos V, Manou V, Kellis E, Kellis S. Body composition characteristics of elite male basketball players. J Hum Mov Stud. 2005;49(2):115-26.
187. Masanovic B, Popovic S, Bjelica D. Comparative study of anthropometric measurement and body composition between basketball players from different competitive levels: elite and sub-elite. Pedagogy Phys Cult Sports. 2019;4.

188. Mathur DN, Toriola AL, Igbokwe NU. Somatotypes of Nigerian athletes of several sports. Br J Sports Med. 1985;19(4):219-20.

189. Muratovic A, Vujovic D, Hadzic R. Comparative study of anthropometric measurement and body composition between elite handball and basketball players. Montenegrin J Sports Sci Med. 2014;3(2):19-22.

190. Popovic S, Akpinar S, Jaksic D, Matic R, Bjelica D, Popovic S, et al. Comparative study of anthropometric measurement and body composition between elite soccer and basketball players. Int J Morphol. 2013;31(2):461-7.

191. Sanfilippo J, Krueger D, Heiderscheit B, Binkley N. Dualenergy X-ray absorptiometry body composition in NCAA Division I Athletes: exploration of mass distribution. Sports Health. 2019;11(5):453-60.

192. Spenst LF, Martin AD, Drinkwater DT. Muscle mass of competitive male athletes. J Sports Sci. 1993;11(1):3-8.

193. Bangsbo J, Iaia FM, Krustrup P. The Yo-Yo intermittent recovery test. Sports Med. 2008;38(1):37-51.

194. Castagna C, Impellizzeri FM, Rampinini E, D’Ottavio S, Manzi $\mathrm{V}$. The Yo-Yo intermittent recovery test in basketball players. J Sci Med Sport. 2008;11(2):202-8.

\section{Authors and Affiliations}

\section{Matthew Morrison ${ }^{1} \cdot$ David T. Martin ${ }^{1} \cdot$ Scott Talpey $^{2} \cdot$ Aaron T. Scanlan $^{3} \cdot$ Jace Delaney $\cdot$ Shona L. Halson $^{1,4}$. Jonathon Weakley ${ }^{1,4,5}$}

Jonathon Weakley

Jonathon.weakley@acu.edu.au

1 School of Behavioural and Health Sciences, Australian Catholic University, Brisbane, QLD, Australia

2 School of Science, Psychology and Sport, Federation University Australia, Ballarat, VIC, Australia

3 Human Exercise and Training Laboratory, School of Health, Medical and Applied Sciences, Central Queensland University, Rockhampton, QLD, Australia
4 Sports Performance, Recovery, Injury and New Technologies (SPRINT) Research Centre, Australian Catholic University, Brisbane, QLD, Australia

5 Carnegie Applied Rugby Research (CARR) Centre, Institute of Sport, Physical Activity and Leisure, Leeds Beckett University, Leeds, UK 